\title{
THE SPACE OF FRAMED FUNCTIONS
}

\author{
KIYOSHI IGUSA
}

\begin{abstract}
We define the notion of a "framed function" on a compact smooth manifold $N$ and we show that the space of all framed functions on $N$ is $(\operatorname{dim} N-$ 1)-connected. A framed function on $N$ is essentially a smooth function $N \rightarrow \mathbf{R}$ with only Morse and birth-death singularities together with certain additional structure.
\end{abstract}

Introduction. The purpose of this paper is to prove that the space of framed functions on a compact smooth manifold $N$ is $(\operatorname{dim} N-1)$-connected. The precise definitions and statements are given in $\$ 1$. The purpose of this introduction is to explain why we are interested in framed functions, in particular we explain how they are related to pseudoisotopy theory.

Suppose that $W^{n+1}$ is a cobordism from $M_{0}$ to $M_{1}$ (all manifolds being compact and smooth). Thus $\partial W=M_{0} \cup M_{1} \cup\left(\partial M_{0} \times I\right)$. Also suppose we have a Morse function $f: W \rightarrow I$. Then we get a finite relative cell complex $X=M_{0} \cup e_{1} \cup e_{2}$ $\cup \cdots \cup e_{m}$ homotopy equivalent to $W$ with one cell for each critical point of $f$, the dimension of the cell being equal to the index of the critical point. There is however some ambiguity in the assignment of $X$ to the function $f$. In order to resolve this ambiguity we add extra structure to the function $f$ and the result is what we call a "framed Morse function."

The first bit of extra structure that we add is a Riemannian metric on $W$ with the appropriate boundary conditions. Given such a metric the cell $e\left(x_{0}\right)$ corresponding to a critical point $x_{0}$ of $f$ can be defined to be the closure of the set of all $y \in W$ so that the trajectory $\psi(t)$ of $\nabla f$ through $y$ converges to $x_{0}$ as $t \rightarrow+\infty$ (aithough we usually use a different more complicated definition for the cells $e(x)$ ).

Given the cells of $X$ as point sets we still need a parametrization of the cells, i.e. for each closed $i$-cell $e^{i}$ we need a map from the standard $i$-disk $D^{i}$ into $X$ with image $e^{i}$. To achieve this it is enough to choose an orthonormal basis for the tangent space of the cells $e(x)$ at each of the critical points $x$ of $f$. The tangent space $T_{x} e(x)$ is the same as the (-) eigenspace of $D^{2} f(x)$ considered as the covariant derivative of $\nabla f$ at $x$.

Received by the editors November 18, 1985. Presented in a talk at the NSF-CBMS Conference on Geometric Topology held at the University of Notre Dame, July 16-27, 1984.

1980 Mathematics Subject Classification (1985 Revision). Primary 57R65; Secondary 57R45.

Key words and phrases. Singularities, smooth manifolds, Morse functions, jet bundles, cell complexes. This research is supported by NSF Grant No MCS-82-02246. 
A framed Morse function on $W$ is defined to be a triple $(f, \mathfrak{m}, \xi)$, where $f$ : $W \rightarrow I$ is a Morse function, $m$ is a Riemannian metric on $W$, and $\xi$ is an orthonormal framing for the (-) eigenspace of $D^{2} f$ at each critical point of $f$. The space of framed Morse functions on $W$ has a natural $C^{\infty}$ topology.

More generally a framed function on $W$ is defined to be a triple $(f, \mathfrak{m}, \xi)$, where $f$ is a generalized Morse function (having only nondegenerate and birth-death singularities) and $\mathfrak{m}, \xi$ are as above. Instead of trying to topologize the space of framed functions we make it into a simplicial set by defining $k$-parameter families of framed functions in a natural way (see $\$ 1$ for details). The main theorem of this paper says that the geometric realization of this simplicial set is $n$-connected $(n=\operatorname{dim} W-1)$.

As in the case of framed Morse functions, to each framed function on $W$ we can associate a finite relative cell complex $X$ homotopy equivalent to $W$. This procedure associates to each birth-death point a pair of cells in "cancelling position." A small perturbation of the framed function will result in either the elementary collapse of these two cells or the "resolution" of the pair of cells into the same but unpaired cells, i.e. an elementary expansion.

We now consider pseudoisotopies. We recall that the pseudoisotopy space of a manifold $M$ is $C(M)=\operatorname{Diff}(M \times I$ rel $M \times 0 \cup \partial M \times I)$. A classifying space for pseudoisotopies can be constructed as follows. Let $E C(M)$ denote the space of all smooth embeddings $g$ : $M \times I \rightarrow M \times[0,2]$ so that $g$ is the inclusion map on "the bottom and sides" $=M \times 0 \cup \partial M \times I=g^{-1} \partial(M \times[0,2])$. Then $E C(M)$ is a contractible space with a free $C(M)$ action. Two embeddings lie in the same orbit if and only if their images coincide. Consequently $B C(M)=E C(M) / C(M)$ is the space of all submanifolds $W$ of $M \times[0,2]$ so that $W$ is diffeomorphic to $M \times I$ and $W \cap \partial(M \times[0,2])=M \times 0 \cup \partial M \times I$.

Now consider $B^{\prime} C(M)=$ the space of all pairs $(W, h)$, where $W \in B C(M)$ and $h$ is a framed function on $W$. Then the framed function theorem implies that a suitable simplicial version of this space has the $\operatorname{dim} M$ homotopy type of $B C(M)$. The cell complex construction gives a map $B^{\prime} C(M) \rightarrow$ "the space of all finite relative cell complexes homotopy equivalent to $M$ " = Waldhausen's "expansion space" [W]. I claim that this map is a homotopy equivalence onto the zero component (the components of the expansion space form a group-namely the Whitehead torsion group for $M$ ) in the "stable range." In fact it is almost circular reasoning to say this since a version of this construction forms part of the proof of the stability theorem [I4].

As I hope to explain in a future joint paper with Friedhelm Waldhausen this construction gives another proof of Waldhausen's formula for stable pseudoisotopies, namely

$$
A(X) \simeq B^{2} P(X) \times Q\left(X_{+}\right),
$$

where $A(X)$ is Waldhausen's algebraic $K$-theory of the space $X, P(X)$ is the stable pseudoisotopy space of $X, B^{2} P(X)$ is a 2-fold delooping of $P(X)$, and $Q=\Omega^{\infty} S^{\infty}$.

Returning to the actual contents of this paper:

$\S 1$ contains the precise definition of the framed function space $\left|\mathscr{L}^{\Delta}(N)\right|$ and shows that it is connected. 
In $\S 2$ we construct the corresponding space of sections of the jet bundle, to wit, the space of "framed sections" and show that it is contractible. The idea of the proof of the framed function theorem will be to compare framed functions with framed sections.

In $\$ 3$ we define " $q$-semiframed functions and sections." When $q=0$ these are framed functions and sections and when $q=n$ these are functions and sections with no framed structure up to homotopy. The main result of [I2] implies that the space of $n$-semiframed functions has the $\operatorname{dim} N$ homotopy type of the space of $n$-semiframed sections. The idea is now to do downward induction on $q$ by showing that the obstruction to making a family of $q$-semiframed functions into $(q-1)$ semiframed functions is the same as the obstruction to making the corresponding $q$-semiframed sections into $(q-1)$-semiframed sections.

$\$ 4$ proves a simplifying lemma for the obstruction set $\Sigma^{q}(h)$ which is the set which must be eliminated in order to make a family $h$ of $q$-semiframed functions into $(q-1)$-semiframed functions.

$\S 5$ proves that certain simplifying deformations (surgeries) of the obstruction set $\Sigma^{q}(\sigma)$ of a $q$-semiframed family of section $\sigma$ can be realized by a deformation of $\sigma$.

$\S 6$ shows the corresponding thing for $q$-semiframed functions.

$\$ 7$ shows that the space of $q$-semiframed functions on $N$ has the $\operatorname{dim} N$ homotopy type of the space of $q$-semiframed sections on $N$ as long as $q>0$, i.e. except in the framed $=0$-semiframed case. The reason that the argument does not work for $q=0$ is because the result in $\S 4$ does not hold when $q=1$.

In $\S 8$ we modify the argument in $\S 4$ so that it applies in the case when $q=1$, thus completing the proof of the main theorem. The new argument uses dovetails and butterflies ( $A_{3}$ and $A_{4}$ singularities respectively) to construct an inadmissible deformation of a family of 1-semiframed functions in order to simplify the obstruction set and thus eliminate it as in \$7. By a simple trick the existence of an inadmissible deformation implies the existence of a good deformation (without $A_{3}$, $A_{4}$ singularities).

Finally there is an appendix which explains those elementary facts about $A_{1}, A_{2}, A_{3}, A_{4}$ singularities which are used in the paper.

1. Statement of the main theorem. Throughout this paper $N$ will denote a compact smooth $\left(C^{\infty}\right) n$-manifold and $g: N \rightarrow \mathbf{R}$ will be a fixed Morse function without critical points on $\partial N$. We shall say that a smooth function $f: N \rightarrow \mathbf{R}$ is admissible if $f=g$ near $\partial N$. We shall consider only admissible functions.

If an admissible function $f: N \rightarrow \mathbf{R}$ has only Morse $\left(A_{1}\right)$ and birth-death $\left(A_{2}\right)$ singularities as defined in the appendix we call it a generalized Morse function. We denote by $\mathscr{H}(N)$ the space of all such functions with the weak $C^{\infty}$ topology.

THEOREM 1.1 [I3]. There is an n-connected map $\mathscr{H}(N) \rightarrow Q\left(B O \wedge\left(N_{+}\right)\right)$, where $Q=\Omega^{\infty} S^{\infty}$ and $N_{+}=N \amalg \mathrm{pt}$.

We will modify the space $\mathscr{H}(N)$ so that an analogous theorem will hold for the new space with $B O$ replaced by $E O \simeq *$. Since $Q\left(* \wedge\left(N_{+}\right)\right)$is contractible, the new space will be $(n-1)$-connected. 
Definition 1.2. A framed function on $N$ is a triple $(f, \mathfrak{m}, \xi)$, where $f \in \mathscr{H}(N)$, $\mathrm{m}$ is a Riemannian metric on $N$, and $\xi$ is an orthonormal framing for the negative eigenspace of the second derivative of $f$ at each critical point. Let $\mathscr{L}(N)$ denote the set of all framed functions on $N$.

Instead of trying to topologize the set $\mathscr{L}(N)$ we shall make it into a simplicial set. Since we want to compare $\mathscr{L}(N)$ with $\mathscr{H}(N)$, we shall also make $\mathscr{H}(N)$ into a simplicial set. Thus let $\mathscr{H}^{\Delta}(N)$ be the simplicial set whose $k$-simplicies are families of maps $f_{t}$ in $\mathscr{H}(N)$ smoothly parameterized by $t \in \Delta^{k}$ and satisfying the following transversality conditions.

(1) The normalized 3-jet $\tilde{j}^{3} f: N \times \Delta^{k} \rightarrow \tilde{J}^{3}(N)$ meets $A_{2}(N)$ transversely.

(2) The $A_{2}$-manifold $A_{2}(f)=\left(\tilde{j}^{3} f\right)^{-1} A_{2}(N)$ is transverse to $N \times \sigma$ for all faces $\sigma$ of $\Delta^{k}$.

The terminology is explained in the appendix. Condition (1) implies that $A_{2}(f)$ is a $(k-1)$-manifold and condition (2) implies that $\tilde{j}^{3} f \mid N \times \sigma$ is also transverse to $A_{2}(N)$. The faces and degeneracies in $\mathscr{H}^{\Delta}(N)$ are defined in the obvious way.

Proposition 1.3. (a) $\mathscr{H}^{\Delta}(N)$ is a Kan complex.

(b) The map $\left|\mathscr{H}^{\Delta}(N)\right| \rightarrow \mathscr{H}(N)$ induced by inclusion is a weak homotopy equivalence.

Proof. (a) This is clear. Let $T=\left\{t \in I^{k} \mid \sum t_{i} \leqslant 1\right\}$. Let $T_{i}=\left\{t \in T \mid t_{i}=0\right\}$. Let $f_{t}$ be a smooth family of functions in $\mathscr{H}(N)$ defined for all $t \in \cup T_{i}$ so that the restriction of $f_{t}$ to each $T_{i}$ satisfies the above transversality conditions. For $t \in T$ let $h_{t}$ be given by $h_{t}=f_{s_{1}}+f_{s_{2}}+\cdots+f_{s_{k}}-(k-1) f_{0}$, where $s_{i}=t-$ $\left(0,0, \ldots, 0, t_{i}, 0, \ldots, 0\right)=(t$ with the $i$ th entry replaced by 0$)$. Then $h_{t}$ is a smooth family of admissible functions on $T$ which agrees with $f_{t}$ on $\cup T_{i}$. Since $\mathscr{H}(N)$ is an open subset of the space of all admissible functions and since transversality is an open condition, $h$, will be a transverse family in $\mathscr{H}(N)$ for $t$ close to $\cup T_{i}$.

Let $\phi: T \rightarrow T$ be an embedding which is the identity on $\cup T_{i}$ whose image lies in a small neighborhood of $U T_{i}$. Then $g_{t}=h_{\phi(t)}$ is an extension of $f_{t}$ to $T$ as desired.

(b) This is a standard type of result that follows from the fact that continuous families of smooth maps can be approximated by smooth families of smooth maps satisfying any countable list of transversality conditions (see e.g. $[\mathbf{H}]$ ).

Let $\mathscr{L}^{\Delta}(N)$ denote the simplicial set whose $k$-simplices are triples $\left(f_{t}, \mathrm{~m}_{t}, \xi_{t}\right)$, where $f_{t} \in \mathscr{H}(N), t \in \Delta^{k}$, is a transverse smooth family as described above, $\mathfrak{m}_{t}$ is a smooth family of metrics on $N$ parameterized by $\Delta^{k}$, and $\xi_{t}$ is a family of framings as described in 1.2 so that $\xi_{t}$ is smooth in the following sense.

Let $U_{i}$ be the set of all $(x, t) \in N \times \Delta^{k}$ so that $x$ is a critical point of $f_{t}$ of index $\geqslant i$. Then the transversality conditions imply that $W_{i}=U_{i} \cup A_{2}^{i-1}(f)$ is a compact smooth $k$-manifold with corners (e.g. $W_{i}$ could be diffeomorphic to $\Delta^{k}$ ). The vector $\xi^{i}$ is defined at each point of $U_{i}$. This can be extended to $W_{i}$ by defining $\xi^{i}$ at $(x, t) \in A_{2}^{i-1}(f)$ to be the unit vector in the kernel of $D^{2} f_{t}(x)$ which points in the positive cubic direction. We say that $\xi_{t}$ is smooth if $\xi^{i}$ is a smooth function on $W_{i}$ for each $i$. 

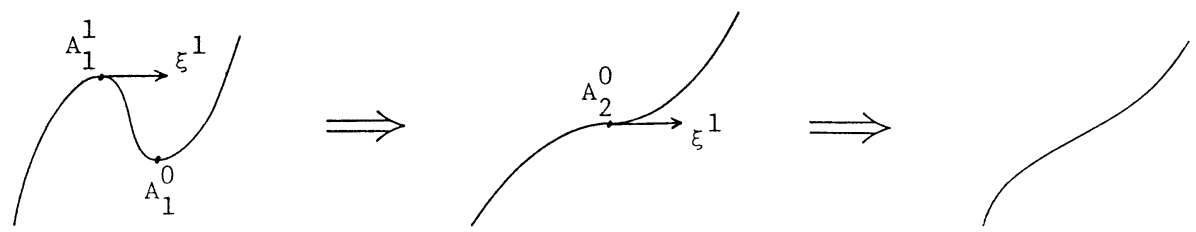

Figure A (cancellation of framed $A_{1}$ points)

As an example suppose that $N=I=[0,1]$ and $(f, \mathfrak{m}, \xi)$ is a framed Morse function on $N$ so that $f$ has one $A_{1}^{0}$ point and one $A_{1}^{1}$ point. Then these two critical points can be cancelled by a path in $\mathscr{L}^{\Delta}(N)$ if $\xi^{1}$ at the $A_{1}^{1}$ point points toward the $A_{1}^{0}$ point (see Figure A).

Proposition 1.4. $\mathscr{L}^{\Delta}(N)$ is a connected Kan complex.

Proof. We first show that $\mathscr{L}^{\Delta}(N)$ is a Kan complex. Let $K$ be the union of all but one of the $(k-1)$-faces of $\Delta^{k}$. Then a simplicial map $K \rightarrow \mathscr{L}^{\Delta}(N)$ is the same as a "transverse smooth map" $K \rightarrow \mathscr{L}(N)$. This consists of a smooth family of framed functions $\left(f_{t}, \mathfrak{m}_{t}, \xi_{t}\right)$ parameterized over $K$ which satisfies the transversality conditions listed above in 1.3. To show that $\mathscr{L}^{\Delta}(N)$ is a Kan complex we need to extend this to a transverse smooth map $\Delta^{k} \rightarrow \mathscr{L}(N)$.

By 1.3(a) we can extend $f_{t}$ to a transverse smooth family $f_{t}^{\prime}$ parameterized over $\Delta^{k}$. Using a partition of unity we can extend $\mathfrak{m}_{t}$ to a smooth family of metrics $\mathrm{m}_{t}^{\prime}$ parameterized over $\Delta^{k}$. Along the $(k-1)$-manifolds $A_{2}^{i-1}\left(f^{\prime}\right) \subset N \times \Delta^{k}$ we can define the vector $\xi^{i \prime}$ to be the unit vector in $\operatorname{ker} D^{2} f^{\prime}$ pointing in the positive cubic direction (using the metric $\mathrm{m}_{t}^{\prime}$ to measure length). For points in $A_{1}^{i}\left(f^{\prime}\right)$ close to $A_{2}^{i-1}\left(f^{\prime}\right)$ we can choose a smoothly varying unit vector $\xi^{i \prime}$ in the negative eigenspace of $D^{2} f^{\prime}$ so that the orthogonal complement of $\xi^{i \prime}$ in the (-) eigenspace of $D^{2} f^{\prime}$ is a smooth continuation of the (-) eigenspace of $D^{2} f^{\prime}$ along $A_{2}^{i-1}\left(f^{\prime}\right) \cup A_{1}^{i-1}\left(f^{\prime}\right)$. At each critical point of $f^{\prime}$ of index $\geqslant i$ we can choose a smoothly varying tangent vector $\xi^{i \prime}$ which agrees with the above construction near $A_{2}^{i-1}\left(f^{\prime}\right)$. For $t$ in a neighborhood of $K$ in $\Delta^{k}$ the projections of these vectors to the (-) eigenspace of $D^{2} f_{t}^{\prime}$ will be linearly independent at each point of $\Sigma\left(f_{t}^{\prime}\right)$. Using the Gram-Schmidt orthogonalization process on these projections we can orthogonalize the vectors $\xi^{i \prime}$ in reverse order starting with $\xi^{n \prime}$. The result will be a smooth family of framings $\xi_{t}^{\prime}$ defined for $t$ in a neighborhood of $K$ in $\Delta^{k}$. As in 1.3(a) let $\phi: \Delta^{k} \rightarrow \Delta^{k}$ be any smooth map which is the identity on $K$ whose image lies in this neighborhood of $K$. Then $\left(f^{\prime}, \mathfrak{m}^{\prime}, \xi^{\prime}\right) \phi: \Delta^{k} \rightarrow \mathscr{L}(N)$ is the desired extension of $(f, \mathfrak{m}, \xi): K \rightarrow \mathscr{L}(N)$.

We shall now show that $\mathscr{L}^{\Delta}(N)$ is connected. For this we take $\left(f_{0}, \mathfrak{m}_{0}, \xi_{0}\right)$ and $\left(f_{1}, \mathfrak{m}_{1}, \xi_{1}\right)$ to be any two vertices of $\mathscr{L}^{\Delta}(N)$, i.e. these are two framed Morse functions on $N$. By 1.1 there is a smooth path $f_{t}, t \in I$, from $f_{0}$ to $f_{1}$ in $\mathscr{H}(N)$. By transversality there will be finitely many birth-death points occurring at different $t$. We may assume there is at most one such point because if there were more we could choose a framing structure for some intermediate $f_{s}$ and break up the deformation $f_{t}$ into two smaller deformations with fewer $A_{2}$ points. 


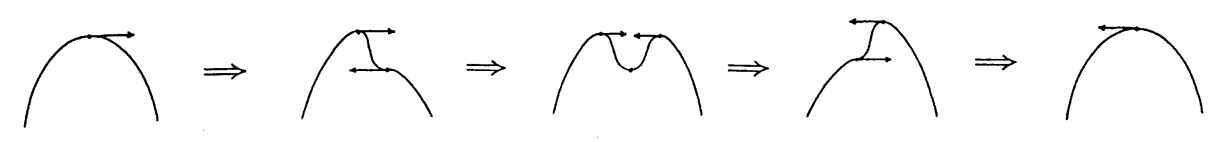

FIGURE B (reversing the framing of an $A_{1}$ point)

If $f_{t}$ has no $A_{2}$ points then we may choose a smooth family of framed structures on $f_{t}$ starting with the given one at $f_{0}$. At $f_{1}$ we get a framed function $\left(f_{1}, \mathrm{~m}_{1}^{\prime}, \xi_{1}^{\prime}\right)$. We can deform $\mathfrak{m}_{1}^{\prime}$ to $\mathfrak{m}_{1}$ and $\xi_{1}^{\prime}$ to $\xi_{1}$ provided that the orientations of $\xi_{1}^{\prime}$ and $\xi_{1}$ agree.

If $f_{t}$ has one birth-death point we may assume by symmetry that it is a "birth" point and that $f_{1}$ has two more critical points than $f_{0}$. Again the framed structure on $f_{0}$ can be smoothly continued to $f_{1}$ and the only obstruction to reaching $\left(f_{1}, \mathfrak{m}_{1}, \xi_{1}\right)$ is the orientation of $\xi_{1}$. Thus it suffices to prove the following lemma.

Lemma 1.5. Let $(f, \mathfrak{m}, \xi)$ be a framed Morse function on $N$ and let $x_{0}$ be a Morse point of $f$ of index $i \geqslant 1$. Let $\xi^{\prime}$ be the same as $\xi$ except for the sign of the ith vector $\xi^{i}$ at $x_{0}$. Then there is a path in $\mathscr{L}^{\Delta}(N)$, i.e. a framed deformation, which connects $(f, \mathfrak{m}, \xi)$ to $\left(f, \mathfrak{m}, \xi^{\prime}\right)$. Furthermore this deformation has support in an arbitrarily small neighborhood $U$ of $x_{0}$. (The support of a deformation is the closure of the set of all points which moves or changes during the deformation.)

Proof. Figure B illustrates the desired deformation in the case $N=I$. In general the desired deformation can be constructed as follows. Let $f_{t}, t \in I$, be the constant deformation $f_{t}=f$. Now apply the negative dovetail lemma (A3.4 or A3.1) and deform $f_{t}$ with support in $U \times(0,1)$ by passing through a $(-)$ dovetail at $\left(x_{0}, 1 / 2\right)$ so that the quartic direction is tangent to $\xi^{i}$. The resulting deformation $f_{t}^{\prime}$ from $f$ to itself has support in $U$ and the graphic indicated in Figure $C$. We have a smooth continuation of the $(i-1)$-framing $\left(\xi^{1}, \ldots, \xi^{i-1}\right)$ at $x_{0}$. To extend this to a framed structure on $f_{t}$ the choice of the $i$ th vector is forced by the two $A_{2}$ points. This gives $\xi$ at one end and $\xi^{\prime}$ at the other. Which end goes where depends on how $\xi^{i}$ is coupled with the deformation parameter $t$.

The main theorem of this paper is the following.

THEOREM 1.6. $\mathscr{L}^{\Delta}(N)$ is $(n-1)$-connected where $n=\operatorname{dim} N$.

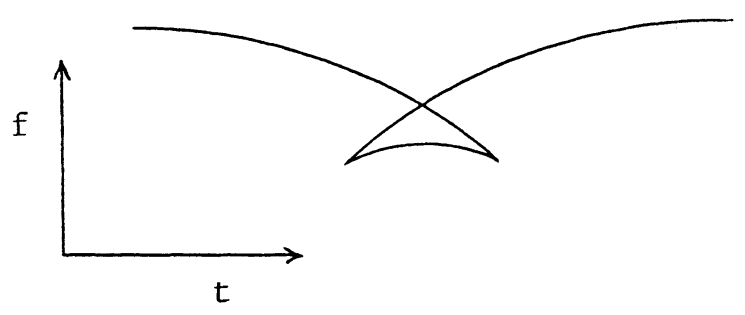

Figure C (graphic of Figure B) 
By Proposition 1.4 we may assume that $n \geqslant 2$.

REMARK 1.7. There are several versions of the space $\left|\mathscr{L}^{\Delta}(N)\right|$ which may be useful for applications.

(1) We could take framed functions $f: N \rightarrow I$.

(2) We could fix the metric on $N$.

(3) We could fix the metric in a neighborhood of $\partial N$.

(4) We could topologize the set $\mathscr{L}(N)$.

Versions (1), (2), and (3) do not change the homotopy type of $\mathscr{L}^{\Delta}(N)$. For version (1) this is trivial (see, e.g. [12, 9.2]). For versions (2) and (3) this follows from the following lemma whose proof is the same as the proof of Lemma 2.9 below.

LEMMA 1.8. Let $\mathscr{I}^{\Delta}(N)$ be the simplicial set whose $k$-simplices are the smooth $\Delta^{k}$-families of metrics on $N$. Then the second coordinate projection map $\mathscr{L}^{\Delta}(N) \rightarrow$ $\mathscr{I}^{\Delta}(N)$ is a Kan fibration.

The following definition for a topology on $\mathscr{L}(N)$ was proposed by Dan Burghelea. We have not checked whether it gives the same weak homotopy type as $\left|\mathscr{L}^{\Delta}(N)\right|$.

Let $\tilde{\mathscr{L}}(N)$ denote the space of all triples $(f, \mathfrak{m}, \xi)$, where $f \in \mathscr{H}(N), \mathfrak{m}$ is a metric on $N$, and $\xi$ is an $n$-tuple of smooth vector fields on $N$ such that $\xi^{1}(x), \ldots, \xi^{i}(x)$ forms an orthonormal basis for the $(-)$ eigenspace of $D^{2} f(x)$ at each critical point $x$ of $f$ of index $i$ and $\xi^{i+1}(x)$ is the unit vector lying in the + cubic direction of $\operatorname{ker} D^{2} f(x)$. We give $\tilde{\mathscr{L}}(N)$ the subspace topology $\tilde{\mathscr{L}}(N) \subset$ $\mathscr{H}(N) \times \mathscr{I}(N) \times \Gamma(T N)^{n}$, where $\mathscr{I}(N)$ is the space of Riemannian metrics on $N$ and $\Gamma(T N)$ is the space of tangent vector fields on $N$. Then let $\mathscr{L}(N)$ be given the quotient topology coinduced by the obvious projection map $\tilde{\mathscr{L}}(N) \rightarrow \mathscr{L}(N)$.

2. Framed sections. In this section we consider "framed" sections of the jet bundle which correspond to framed functions and we show that the space of all framed sections on $N$ is contractible.

We begin with some notational conventions:

$$
\begin{aligned}
& \Sigma(n)=\left\{p \in \tilde{J}^{3}(n) \mid D p(0)=0\right\}, \\
& A_{k}(n)=\left\{p \in \Sigma(n) \mid 0 \text { is an } A_{k} \text { point of } p\right\}, \\
& A(n)=A_{1}(n) \cup A_{2}(n), \\
& H(n)=\left\{p \in \tilde{J}^{3}(n) \mid D p(0) \neq 0 \text { or } p \in A(n)\right\} .
\end{aligned}
$$

Let $\Sigma(N), A_{k}(N), A(N), H(N)$ denote the corresponding subbundles of the normalized jet bundle $\tilde{J}^{3}(N)$ (see A0). Note that $\tilde{J}^{3}(n)-\Sigma(n)=H(n)-A(n) \simeq$ $S^{n-1}$ and $H(n) \simeq S^{n-1} * A(n)$, where $*$ means join.

If $f \in \mathscr{H}(N)$, then $\tilde{j}^{3} f$ is a section of the bundle $H(N)$ which agrees with $\tilde{j}^{3} g$ near $\partial N$. We define $\Gamma_{\mathscr{H}}$ to be the space of all smooth sections of $H(N)$ which agree with $\tilde{j}^{3} g$ near $\partial N$.

THEOREM 2.1 [12]. The normalized 3-jet gives an n-connected map

$$
\tilde{j}_{\mathscr{H}}^{3}: \mathscr{H}(N) \rightarrow \Gamma_{\mathscr{H}} .
$$


There is an obvious notion of a framed structure for a section of $H(N)$ :

Definition 2.2. A framed section of $H(N)$ is a triple $(\sigma, \mathfrak{m}, \xi)$, where $\sigma \in \Gamma_{\mathscr{H}}, \mathfrak{m}$ is a Riemannian metric on $N$, and $\xi$ is a framing of the negative eigenspace of $D^{2} \sigma$ at each point of $\Sigma(\sigma)=\sigma^{-1} \Sigma(N)=$ the singular set of $\sigma$. The set of all framed sections is denoted $\Gamma_{\mathscr{L}}$.

To each framed function $(f, \mathfrak{m}, \xi)$ we can associate the framed section $\left(\tilde{j}^{3} f, \mathfrak{m}, \xi\right)$. This gives a set map $\tilde{j}_{\mathscr{L}}^{3}: \mathscr{L}(N) \rightarrow \Gamma_{\mathscr{L}}$. To avoid questions of general topology we will use simplicial versions of $\Gamma_{\mathscr{H}}$ and $\Gamma_{\mathscr{L}}$.

Let $\Gamma_{\mathscr{H}}^{\Delta}$ denote the simplicial set whose $k$-simplices are smoothly parameterized families of sections $\sigma_{t} \in \Gamma_{\mathscr{H}}, t \in \Delta^{k}$, which are transverse in the following sense.

(1) $\sigma: N \times \Delta^{k} \rightarrow H(N)$ is transverse to $\Sigma(N)$ and to $A_{2}(N)$.

(2) The manifolds $\Sigma(\sigma)=\sigma^{-1} \Sigma(N)$ and $A_{2}(\sigma)=\sigma^{-1} A_{2}(N)$ are transverse to $N \times \tau$ for every face $\tau$ of $\Delta^{k}$.

Proposition 2.3. (a) $\Gamma_{\mathscr{H}}^{\Delta}$ is a Kan complex.

(b) The map $\left|\Gamma_{\mathscr{H}}^{\Delta}\right| \rightarrow \Gamma_{\mathscr{H}}$ induced by inclusion is a weak homotopy equivalence.

(c) The normalized 3-jet gives an n-connected simplicial map

$$
\tilde{j}_{\mathscr{H}}^{\Delta}: \mathscr{H}^{\Delta}(N) \rightarrow \Gamma_{\mathscr{H}} .
$$

Proof. (a) is trivial (see 1.3(a)).

(b) is a standard type of theorem like 1.3(b) (see $[\mathbf{H}]$ ).

(c) follows from Proposition 1.3, Definition 2.2, and (b).

Let $\Gamma_{\mathscr{L}}^{\Delta}$ denote the simplicial set whose $k$-simplices are triples $\left(\sigma_{t}, \mathfrak{m}_{t}, \xi_{t}\right)$, where $\sigma_{t}$ is as above, $\mathfrak{m}_{t}$ is a smooth family of metrics on $N$, and $\xi_{t}$ is a system of framings which is smooth in the same sense as in the definition of $\mathscr{L}^{\Delta}(N)$. Let $\tilde{j}_{\mathscr{L}}^{\Delta}$ : $\mathscr{L}^{\Delta}(N) \rightarrow \Gamma_{\mathscr{L}}^{\Delta}$ be the simplicial map induced by $\tilde{j}_{\mathscr{L}}^{3}$. Then we shall prove the following theorem.

THEOREM 2.4. $\Gamma_{\mathscr{L}}^{\Delta}$ is contractible.

COROLlaRy 2.5. (a) The map $\mathscr{L}^{\Delta}(N) \rightarrow \mathscr{H}^{\Delta}(N)$ is trivial on $\pi_{k-1}$ for $k \leqslant n$.

(b) $\mathscr{L}^{\Delta}(N)$ is $(n-1)$-connected if the following square is $(n+1)$-connected:

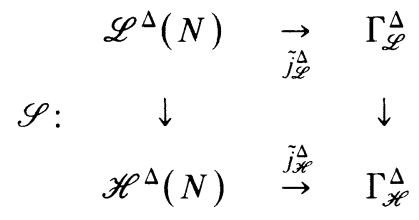

Proof. (a) Look at $\mathscr{S}$ and apply 2.3(b) and 2.4.

(b) If $\mathscr{S}$ is $(n+1)$-connected then the induced map hofib $\tilde{j}_{\mathscr{L}}^{\Delta} \rightarrow$ hofib $\tilde{j}_{\mathscr{H}}^{\Delta}$ is $n$-connected. But hofib $\tilde{j}_{\mathscr{L}}^{\Delta} \simeq \mathscr{L}^{\Delta}(N)$ by 2.4 and hofib $\tilde{j}_{\mathscr{H}}^{\Delta}$ is $(n-1)$-connected by 2.3(b). (Here hofib = homotopy fiber.)

The rest of this section deals with the proof of 2.4. The idea is to construct an $O(n)$-space $L(n)$ so that $\Gamma_{\mathscr{L}}^{\Delta}$ is weakly equivalent to the space of all admissible sections of the corresponding bundle $L(N)$. Theorem 2.4 then becomes equivalent to Lemma 2.8 which says that $L(n)$ is weakly contractible. 
Roughly speaking the space $L(n)$ is constructed by replacing the subspace $A(n)$ of $H(n)$ by a larger space $C(n)$. The elements of $C(n)$ can be viewed as pairs $(p, \xi)$, where $p \in A(n)$ and $\xi$ is an orthonormal framing for the (-) eigenspace of $D^{2} p(0)$. A more precise decription of $L(n)$ is given as follows. We construct an $O(n)$-invariant tubular neighborhood $K(n)$ of $A(n)$ in $H(n)$, where $K(n) \cong D^{n} \times A(n)$, and we let

$$
L(n)=(H(n)-\operatorname{int} K(n)) \cup_{\eta}\left(D^{n} \times C(n)\right)
$$

where $\eta: S^{n-1} \times C(n) \rightarrow S^{n-1} \times A(n) \subset \partial K(n) \subset H(n)-$ int $K(n)$ is an obvious map. A precise definition of $C(n)$ follows the proof of 2.6.

LEMMA 2.6. There is an arbitrarily small $O(n)$-invariant tubular neighborhood $K(n)$ of $A(n)$ in $H(n)$ and an $O(n)$-equivariant diffeomorphism $\phi: K(n) \cong D^{n} \times A(n)$.

REMARK. Assume that a fixed metric $\mathrm{m}_{0}$ for $N$ has been chosen. Then, as we explain in the appendix (A0), $\tilde{J}^{3}(N)$ and $H(N)$ become $O(n)$-bundles, $K(n)$ determines a subbundle $K(N)$ of $H(N)$, and $\phi$ gives a bundle isomorphism $\Phi$ : $K(N) \cong D^{N} \oplus A(N)$, where $D^{N}$ is the tangent disk bundle of $N$ and $\oplus$ denotes fiber product.

Proof. The set of homogeneous polynomials of degree $s$ form an $O(n)$-invariant subset of $\tilde{J}^{3}(n)$. Thus we have an $O(n)$-equivariant isomorphism $\tilde{J}^{3}(n) \cong \tilde{J}^{1}(n) \times$ $\Sigma(n)$. Let $\alpha: A(n) \rightarrow(0, \infty]$ be a proper continuous function with image in $(0, \infty)$. Then the set of all pairs $(x, y) \in H(n) \subset \tilde{J}^{1}(n) \times \Sigma(n)$ with $y \in A(n)$ and $\|x\|<$ $\alpha(y)$ is an arbitrarily small open neighborhood of $A(n)$ in $H(n)$. Since:

(a) $A(n)$ is locally compact,

(b) $O(n)$ is compact, and

(c) $O(n)$ acts smoothly on $A(n)$

there exists a smooth proper function $\beta: A(n) \rightarrow(0, \infty]$ which is strictly smaller than $\alpha$ so that $\infty$ is constant on $O(n)$ orbits. To see this, note that by (a) and (b) the function $\bar{\alpha}$ which takes $y \in A(n)$ to $\inf \alpha(y O(n))$ is lower semicontinuous. Using a partition of unity we can construct a smooth function $\gamma: A(n) \rightarrow(0, \infty]$ which is strictly smaller than $\bar{\alpha}$. Averaging over the action of $O(n)$ we get $\beta$.

Let $K(n)$ be the subset of $H(n) \subset \tilde{J}^{1}(n) \times \Sigma(n)$ consisting of pairs $(x, y)$, where $y \in A(n)$ and $\|x\| \leqslant \beta(y)$. Then $K(n)$ is a closed $O(n)$-invariant submanifold of $H(n)$ and an $O(n)$-equivariant diffeomorphism $\phi: K(n) \cong D^{n} \times A(n)$ can be given by $\phi(x, y)=(x / \beta(y), y)$.

Let $X$ denote the subspace of $A(n) \times O(n)$ consisting of pairs $(p, \xi)$ satisfying the following.

(a) If $p$ has index $i$, then $\xi^{1}, \ldots, \xi^{i}$ form a basis for the (-) eigenspace of $D^{2} p(0)$.

(b) If $p \in A_{2}^{i}(n)$, then $\xi^{i+1}$ lies in the zero eigenspace of $D^{2} p(0)$ and points in the positive cubic direction.

We define an equivalence relation on $X$ by $(p, \xi) \sim\left(p^{\prime}, \xi^{\prime}\right)$ if $p=p^{\prime}$ and $\xi^{j}=\xi^{j \prime}$ for $j \leqslant$ index of $p$. (If $p \in A_{2}^{i}(n)$, then $\xi^{i+1}=\xi^{i+1}$ by (b).) Let $C(n)$ be the quotient space of $X$ via this equivalence relation. Note that the projection map $X \rightarrow A(n)$ induces a continuous map $q: C(n) \rightarrow A(n)$. 
LEMMA 2.7. $C(n)$ is weakly contractible.

Proof. This will be a special case of 2.10 below.

Now let $K(n), K(N), \phi, \Phi$ be as given in 2.6. Let $L(n)$ be the quotient space of $\left(D^{n} \times C(n)\right) \cup(H(n)-$ int $K(n))$ given by identifying $(x, y) \in S^{n-1} \times C(n)$ with $\phi^{-1}(x, q(y)) \in \partial K(n) \subset(H(n)-$ int $K(n))$.

LEMMA 2.8. $L(n)$ is weakly contractible.

Proof. $L(n)$ is the push-out in the following diagram:

$$
\begin{array}{ccc}
S^{n-1} \times C(n) & \rightarrow & D^{n} \times C(n) \\
\downarrow \phi^{-1}(1, q) & & \downarrow \\
H(n)-\operatorname{int} K(n) & \rightarrow & L(n)
\end{array}
$$

By 2.7 we have $D^{n} \times C(n) \simeq *$ and $S^{n-1} \times C(n) \simeq S^{n-1}$. On the other hand $H(n)$ - int $K(n) \simeq H(n)-A(n) \cong\left(J^{1}(n)-0\right) \times \Sigma(n) \simeq S^{n-1}$ so the map $\phi^{-1}(1, q)$ is a weak equivalence.

Note that $L(n)$ is an $O(n)$-space and the obvious projection $L(n) \rightarrow H(n)$ is $O(n)$-equivariant. This means that $L(n)$ determines an $O(n)$ bundle $L(N)$ over $N$ and a bundle map $L(N) \rightarrow H(N)$. It follows from 2.8 that the space of sections of $L(N)$ is weakly contractible.

Let $\mathscr{I}^{\Delta}(N)$ denote the simplicial set whose $k$-simplices are smooth $\Delta^{k}$-families of metrics on $N$.

LEMMA 2.9. $\mathscr{I}^{\Delta}(N)$ and $\Gamma_{\mathscr{L}}^{\Delta}$ are Kan complexes, $\mathscr{I}^{\Delta}(N)$ is contractible and the second coordinate map $\Gamma_{\mathscr{L}}^{\Delta} \rightarrow \mathscr{I}^{\Delta}(N)$ is a Kan fibration.

Assume for a moment that this lemma is true. Then we have

Proof of TheOrem 2.4. A simplicial map $\partial \Delta^{k} \rightarrow \Gamma_{\mathscr{L}}^{\Delta}$ is equivalent to a "transverse smooth map" $\partial \Delta^{k} \rightarrow \Gamma_{\mathscr{L}}$. This consists of a smooth family of framed sections $\left(\sigma_{t}, m_{t}, \xi_{t}\right)$ parameterized over $\partial \Delta^{k}$ which satisfies the transversality conditions listed above in 2.3. To prove 2.4 we must show that every such map extends to a transverse smooth map $\Delta^{k} \rightarrow \Gamma_{\mathscr{L}}$.

Using 2.9 we may assume that for all $t \in \partial \Delta^{k}, \mathfrak{m}_{t}=\mathfrak{m}_{0}$ is a fixed metric on $N$ and we may assume that $\mathfrak{m}_{0}$ was the metric used to reduce the structure group of $\tilde{J}^{3}(N)$ to $O(n)$ and to construct $K(N)$.

If $K(N)$ is sufficiently small, then $K(\sigma)$ which is defined to be $\sigma^{-1} K(N) \subset N \times$ $\partial \Delta^{k}$ will be a closed tubular neighborhood of $\Sigma(\sigma)=\sigma^{-1} \Sigma(N)=\sigma^{-1} A(N)$. Since the inclusion $K(\sigma) \subset N \times \partial \Delta^{k}$ is a cofibration, we may deform $\sigma$ fixing $\sigma \mid \Sigma(\sigma)$ so that the restriction of $\sigma$ to $K(\sigma)$ is a bundle map $K(\sigma) \rightarrow K(N)$ over the fixed map $\sigma \mid \Sigma(\sigma): \Sigma(\sigma) \rightarrow A(N)$. Using $\xi_{t}$ we can lift this bundle map to $D^{N} \oplus C(N)$ and thus get a map $N \times \partial \Delta^{k} \rightarrow L(N)$ lifting $\sigma$. This gives a lifting of $\partial \Delta^{k} \rightarrow \Gamma^{\Delta}$ to the contractible simplicial set $\Gamma_{L}^{\Delta}=$ the simplicial set of $\Delta^{k}$-families of sections of $L(N)$ which are fixed near $\partial N$. Note that the space $\Gamma_{L}^{\Delta}$ depends on the choice of $\mathfrak{m}_{0}$ and $K(N)$. This completes the proof of 2.4 modulo Lemmas 2.7 and 2.9.

Proof of 2.9. It is clear that $\mathscr{I}^{\Delta}(N)$ is a contractible Kan complex. An argument similar to the proof of 1.4 shows that $\Gamma_{\mathscr{L}}^{\Delta}$ is a Kan complex. So it remains to show that $\Gamma_{\mathscr{L}}^{\Delta} \rightarrow \mathscr{I}^{\Delta}(N)$ is a Kan fibration. 
Let $K$ be the union of all but one of the $(k-1)$-faces of $\Delta^{k}$ and let $\left(\sigma_{t}, \mathfrak{m}_{t}, \xi_{t}\right)$, $t \in K$, be a transverse smooth map $K \rightarrow \Gamma_{\mathscr{L}}$ or equivalently a simplicial map $K \rightarrow \Gamma_{\mathscr{L}}^{\Delta}$. Let $\mathfrak{m}_{t}^{\prime}, t \in \Delta^{k}$, be a $k$-simplex in $\mathscr{I}^{\Delta}(N)$ so that $\mathfrak{m}_{t}^{\prime}=\mathfrak{m}_{t}$ for all $t \in K$. Since $\Gamma_{\mathscr{L}}^{\Delta}$ is a Kan complex there exists an extension of $\left(\sigma_{t}, \mathfrak{m}_{t}, \xi_{t}\right)$ to $\Delta^{k}$ which we denote $\left(\sigma_{t}^{\prime \prime}, m_{t}^{\prime \prime}, \xi_{t}^{\prime \prime}\right)$. We shall find a system of framings $\xi_{t}^{\prime}, t \in \Delta^{k}$, so that $\left(\sigma_{t}^{\prime \prime}, m_{t}^{\prime}, \xi_{t}^{\prime}\right), t \in \Delta^{k}$, is a $k$-simplex of $\Gamma_{\mathscr{L}}^{\Delta}$ and $\xi_{t}^{\prime}=\xi_{t}=\xi_{t}^{\prime \prime}$ for all $t \in K$.

We construct the framings $\xi_{t}^{i \prime}$ by induction on $n-i$. To construct $\xi_{t}^{n \prime}$ we start with $\xi_{t}^{n \prime \prime}$ and do the following.

(1) Divide $\xi_{t}^{n \prime \prime}$ by its length with respect to $m_{t}^{\prime}$.

(2) Deform the resulting vector $\xi_{t}^{n \prime \prime} /\left\|\xi_{t}^{n \prime \prime}\right\|$ in a small neighborhood of $A_{2}^{n-1}\left(\sigma^{\prime \prime}\right)$ to a unit vector $\xi_{t}^{n \prime}$ satisfying the following.

(a) The orthogonal complement of $\xi_{t}^{n \prime}$ is a smooth continuation of the (-) eigenspace of $D^{2} \sigma^{\prime \prime}$ along $A_{1}^{n-1}\left(\sigma^{\prime \prime}\right)$.

(b) $\xi_{t}^{n \prime}$ is linearly independent from $\xi_{t}^{1 \prime \prime}, \ldots, \xi_{t}^{n-1 \prime \prime}$. To construct $\xi_{t}^{i \prime}$ we first project $\xi_{t}^{i \prime \prime}$ to the orthogonal complement of the span of $\xi_{t}^{i+1}, \ldots, \xi_{t}^{n \prime}$ in the nonpositive eigenspace of $D^{2} \sigma^{\prime \prime}$, then we perform the analogues of (1), (2) above (normalize and then perturb near $A_{2}^{i-1}\left(\sigma^{\prime \prime}\right)$ to make the orthogonal complement of $\xi_{t}^{i \prime}$ in the (-) eigenspace of $D^{2} \sigma^{\prime \prime}$ the smooth continuation of the (-) eigenspace of $D^{2} \sigma^{\prime \prime}$ along $\left.A_{1}^{i-1}\left(\sigma^{\prime \prime}\right)\right)$.

We shall now prove a generalization of Lemma 2.7 which we will need later. If $0 \leqslant q \leqslant n$ let $W_{q}(n)=A_{1}^{q}(n) \cup A_{2}^{q}(n) \cup A_{1}^{q+1}(n) \cup \cdots \cup A_{1}^{n}(n)=$ the set of all points in $A(n)$ with index $\geqslant q$. Let $X_{q}$ denote the space of all pairs $(p, \xi) \in W_{q}(n)$ $\times\left(\mathbf{R}^{n}\right)^{n-q}$, where we write $\xi=\left(\xi^{q+1}, \ldots, \xi^{n}\right)$, so that:

(a) $\xi$ is orthonormal.

(b) If $p$ has index $i$, then $\xi^{q+1}, \ldots, \xi^{i}$ lie in the $(-)$ eigenspace of $D^{2} p(0)$ and $\xi^{i+1}, \ldots, \xi^{n}$ lie in the $(+, 0)$-eigenspace of $D^{2} p(0)$.

(c) If $p \in A_{2}^{i}(n)$, then $\xi^{i+1}$ lies in the 0 -eigenspace of $D^{2} p(0)$ and points in the + cubic direction.

Define an equivalence relation on $X_{q}$ by $(p, \xi) \sim\left(p^{\prime}, \xi^{\prime}\right)$ if $p=p^{\prime}$ and $\xi^{j}=\xi^{j \prime}$ for $q+1 \leqslant j \leqslant$ index of $p$. Let $C_{q}(n)$ be the quotient space of $X_{q}$ by this equivalence relation.

Proposition 2.10. The map $C_{q}(n) \stackrel{s}{\rightarrow} O(n) /(O(q) \times O(n-q))$ which maps $[p, \xi]$ to the span of $\xi$ is a weak homotopy equivalence.

Note that $C(n)=C_{0}(n)$ so Lemma 2.7 is a special case of this proposition.

Proof. Let $\pi: C_{q}(n) \rightarrow W_{q}(n)$ be the map induced by the projection of $X_{q}$ to $W_{q}(n)$. Let $Y_{q}^{i}=\pi^{-1} A_{2}^{i}(n)$ and let $Z_{q}^{i}=Y_{q}^{i} \cup Y_{q}^{i-1} \cup \pi^{-1} A_{1}^{i}(n)$. These are closed subsets of $C_{q}(n)$ since $\pi$ is continuous. Thus it suffices to show the following.

(1) The $\operatorname{map} Z_{q}^{q} \stackrel{s}{\rightarrow} O(n) /(O(q) \times O(n-q))$ is a homotopy equivalence.

(2) If $i \geqslant q+1$, then $Y_{q}^{i-1}$ is a weak deformation retract of $Z_{q}^{i}$.

We prove (1) first. If $[p, \xi] \in Z_{q}^{q}$, then $\xi=\xi(p)$ is determined (up to equivalence) by $p$. Thus $Z_{q}^{q}$ is homeomorphic to $A_{1}^{q}(n) \cup A_{2}^{q}(n)$. For each $T \in O(n)$ let $P_{T} \in$ $A_{1}^{q}(n)$ be given by $P_{T}(x)=P_{I}\left(T^{-1} x\right)$, where

$$
P_{I}(y)=-y_{1}^{2}-y_{2}^{2}-\cdots-y_{q}^{2}+y_{q+1}^{2}+\cdots+y_{n}^{2} .
$$


Then $P_{T}$ depends only on $\bar{T}=$ the image of $T$ in $O(n) /(O(q) \times O(n-q))$ and $s P_{T}=\bar{T}$. Thus $P$ gives a section of $s$ and a deformation retraction of $Z_{q}^{q}$ to the image of $P$ is given by $\left[p_{t}, \xi\left(p_{t}\right)\right]$, where $p_{t}=t P_{s(p)}+(1-t) p$.

We now prove (2). Let $L \subset K$ be a compact pair of spaces and let $f:(K, L) \rightarrow$ $\left(Z_{q}^{i}, Y_{q}^{i-1}\right)$ be a continuous map. Then we must deform $f$ into $Y_{q}^{i-1}$ fixing $f \mid L$. The deformation will be a straight line deformation from $f$ to a map $g: K \rightarrow Y_{q}^{i-1}$ whose restriction to $L$ is the same as $f$. The map $g$ is given as follows.

For each $u \in K$ let $f(u)=\left(f_{2}(u)+f_{3}(u), f_{1}(u)\right)$, where $f_{2}(u), f_{3}(u)$ are homogeneous polynomials of degree 2,3 respectively. In the special case where $f_{1}(u)^{i}=e_{1}$ $=(1,0, \ldots, 0)$ we want $g(u)$ to be given by $g(u)=\left(g_{2}(u)+g_{3}(u), f_{1}(u)\right)$, where $g_{2}(u)=f_{2}-$ all monomials involving $x_{1}$ (i.e. $g_{2}(u)(x)=f_{2}(u)\left(x-x_{1} e_{1}\right)$ ) and $g_{3}(u)(x)=f_{3}(u)(x)+b(u) x_{1}^{3}$, where $b(u)$ is a real number which is large enough so that $g_{2}(u)+g_{3}(u)$ is an $A_{2}^{i-1}$ singularity with $d x_{1}=e_{1}$ being the + cubic direction of the 0-eigenspace of $D^{2} g(u)(0)$.

In general we must replace $x_{1}$ by the coordinate function corresponding to the unit vector $f_{1}(u)^{i}$. This is given by $x \mapsto\left(x \cdot f_{1}(u)^{i}\right)$. Thus we let $g(u)=\left(g_{2}(u)+\right.$ $\left.g_{3}(u), f_{1}(u)\right)$, where

$$
\begin{aligned}
& g_{2}(u)(x)=f_{2}(u)\left(x-\left(x \cdot f_{1}(u)^{i}\right) f_{1}(u)^{i}\right), \\
& g_{3}(u)(x)=f_{3}(u)(x)+b(u)\left(x \cdot f_{1}(u)^{i}\right)^{3} .
\end{aligned}
$$

The function $b:(K, L) \rightarrow(\mathbf{R}, 0)$ is given as follows. Let $a: K \rightarrow \mathbf{R}$ be the continuous function given by:

$$
a(u)=\frac{1}{6} \frac{d^{3}}{d t^{3}}\left[f_{3}(u)\left(t f_{1}(u)^{i}\right)\right]_{t=0} .
$$

Then $a(u)>0$ for all $u \in L$ so there exists a continuous function $b:(K, L) \rightarrow(\mathbf{R}, 0)$ so that $b(u)>-a(u)$ for all $u \in K$.

The deformation of $f$ to $g$ is given by $\left(t\left(g_{2}+g_{3}\right)+(1-t)\left(f_{2}+f_{3}\right), f_{1}\right)$.

3. Semiframed functions. We introduce some intermediary spaces which lie between $\mathscr{H}^{\Delta}(N)$ and $\mathscr{L}^{\Delta}(N)$.

Definition 3.1. A $q$-semiframed function on $N$ is a triple $(f, \mathfrak{m}, \xi)$, where $f \in \mathscr{H}(N), \mathfrak{m}$ is a Riemannian metric on $N$, and $\xi$ associates to each critical point $x$ of $f$ an ordered set of $i$ vectors in the (-) eigenspace of $D^{2} f(x)$, where $i$ is the index of $f$ at $x$ so that the last $i-q$ vectors of $\xi$ at $x$ are orthonormal. Let $\mathscr{L}_{q}(N)$ denote the set of all $q$-semiframed functions on $N$.

Note that we have a sequence of inclusions: $\mathscr{L}(N)=\mathscr{L}_{0}(N) \subset \mathscr{L}_{1}(N) \subset \cdots \subset$ $\mathscr{L}_{n}(N)$.

Definition 3.2. Let $\mathscr{L}_{q}^{\Delta}(N)$ denote the simplicial set whose $k$-simplices are smooth families of $q$-semiframed functions $\left(f_{t}, \mathfrak{m}_{t}, \xi_{t}\right), t \in \Delta^{k}$, satisfying the same conditions as in the definition of $\mathscr{L}^{\Delta}(N)$ plus one additional condition ((4) below). Thus

(1) $\tilde{j}^{3} f$ is transverse to $A_{2}(N)$ over every face of $\Delta^{k}$.

(2) $\xi_{t}$ varies smoothly with $t \in \Delta^{k}$. 
(3) $\xi^{i}$ is a smooth function on its extended domain $\bar{W}_{i}(f)=\left(\tilde{j}^{3} f\right)^{-1} \bar{W}_{i}(N)$, where $\bar{W}_{i}=W_{i} \cup A_{2}^{i-1}=A_{2}^{i-1} \cup A_{1}^{i} \cup A_{2}^{i} \cup \cdots \cup A_{1}^{n}$.

(4) At each $(x, t)$ in $W_{q}(f)$ take the orthogonal complement of the span of $\xi_{t}^{q+1}$, $\xi_{t}^{q+1}, \ldots$ in the $(-, 0)$-eigenspace of $D^{2} f_{t}$. We require that this $q$-plane vary smoothly with $(x, t)$.

Note that we have a sequence of inclusions: $\mathscr{L}^{\Delta}(N)=\mathscr{L}_{0}^{\Delta}(N) \subset \mathscr{L}_{1}^{\Delta}(N) \subset \cdots$ $\subset \mathscr{L}_{n}^{\Delta}(N)$.

DEFINITION 3.3. A $q$-semiframed section of $H(N)$ is a triple $(\sigma, \mathfrak{m}, \xi)$, where $\sigma \in \Gamma_{\mathscr{H}}, \mathfrak{m}$ is a metric on $N$, and $\xi$ is an $i$-tuple of vectors in the $(-)$ eigenspace of $D^{2} \sigma$ at each point of $\Sigma(\sigma)$ of index $i$ so that the last $i-q$ vectors of $\xi$ are orthonormal. Let $\Gamma_{q}$ denote the set of all $q$-semiframed sections of $H(N)$ and let $\Gamma_{q}^{\Delta}$ denote the corresponding simplicial set defined analogously to 3.2 .

Let $\tilde{j}_{q}^{\Delta}: \mathscr{L}_{q}^{\Delta}(N) \rightarrow \Gamma_{q}^{\Delta}$ be the simplicial map given by $\tilde{j}_{q}^{\Delta}\left(f_{t}, \mathfrak{m}_{t}, \xi_{t}\right)=$ $\left(\tilde{j}^{s} f_{t}, \mathfrak{m}_{t}, \xi_{t}\right)$. Then we have the following commuting diagram:

$$
\begin{aligned}
& \mathscr{L}^{\Delta}(N) \stackrel{=}{\rightarrow} \mathscr{L}_{0}^{\Delta}(N) \stackrel{\ulcorner}{\rightarrow} \mathscr{L}_{1}^{\Delta}(N) \stackrel{\ulcorner}{\rightarrow} \cdots \stackrel{\subset}{\rightarrow} \mathscr{L}_{n}^{\Delta}(N) \rightarrow \mathscr{H}^{\Delta}(N)
\end{aligned}
$$

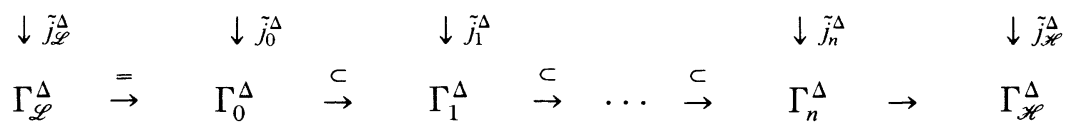

Proposition 3.5. (a) $\mathscr{L}_{q}^{\Delta}(N)$ and $\Gamma_{q}^{\Delta}$ are Kan complexes.

(b) The maps $\mathscr{L}_{n}^{\Delta}(N) \rightarrow \mathscr{H}^{\Delta}(N)$ and $\Gamma_{n}^{\Delta} \rightarrow \Gamma_{\mathscr{H}}^{\Delta}$ given by projection onto the first coordinate are homotopy equivalences.

Proof. (a) is analogous to Proposition 1.4.

(b) It is clear that these maps are onto. To show that the relative homotopy groups are zero we take a smooth family $f_{t}$ in $\mathscr{H}^{\Delta}(N)$ or $\sigma_{t}$ in $\Gamma_{\mathscr{H}}^{\Delta}$ parameterized over $\Delta^{k}$ with an $n$-semiframed structure over $\partial \Delta^{k}$. But there is no obstruction to extending the $n$-semiframed structure to all of $\Delta^{k}$.

Now let $\tau:\left(\Delta^{k}, \partial \Delta^{k}\right) \rightarrow\left(\Gamma_{q}^{\Delta}, \Gamma_{q-1}^{\Delta}\right)$ be a simplicial map. This means we have a smooth family of $q$-semiframed sections $\tau(t)=\left(\sigma_{t}, \mathfrak{m}_{t}, \xi_{t}\right)$ parameterized over $\Delta^{k}$ which are $(q-1)$-semiframed over $\partial \Delta^{k}$. Let $\bar{W}_{q}(\sigma)=W_{q}(\sigma) \cup A_{2}^{q-1}(\sigma)$, where $W_{q}=A_{1}^{q} \cup A_{2}^{q} \cup A_{1}^{q+1} \cup \cdots \cup A_{1}^{n}$ as in $\S 2$. Then $\bar{W}_{q}(\sigma)$ is a compact smooth $k$-manifold with corners with $\bar{W}_{q}(\sigma)=A_{2}^{q-1}(\sigma) \cup\left(\bar{W}_{q}(\sigma)\left(N \times \partial \Delta^{k}\right)\right)$. Let $B_{q}(N) \stackrel{\pi}{\rightarrow} N$ be the bundle of tangent $q$-planes in $N$. If $(x, t) \in W_{q}(\sigma) \subset N \times \Delta^{k}$, then let $\rho_{0}(x, t) \in \pi^{-1}(x)$ be the orthogonal complement of the span of $\left\{\xi_{t}^{i}(x) \mid i>\right.$ $q$ \} in the $(-, 0)$-eigenspace of $D^{2} \sigma_{t}(x)$. Then $\rho_{0}$ is smooth by Definition 3.2(4). Let $\rho: \bar{W}_{q}(\sigma) \rightarrow B_{q}(N) \times \Delta^{k}$ be the map given by $\rho(x, t)=\left(\rho_{0}(x, t), t\right)$. Then the composition $\stackrel{q}{W}_{q}(\sigma) \stackrel{\rho}{\rightarrow} B_{q}(N) \times \Delta^{k} \stackrel{\pi \times 1}{\rightarrow} N \times \Delta^{k}$ is the inclusion map, so $\rho$ is a section of $B_{q}(N) \times \Delta^{k}$ over $\bar{W}_{q}(\sigma)$.

For each $(x, t) \in \bar{W}_{q}(\sigma)$ we can project the vector $\xi_{t}^{q}(x)$ to the plane $\rho_{0}(x, t)$. This gives a lifting $\tilde{\rho}$ of $\rho$ to $E_{q}(N) \times \Delta^{k}$, where $E_{q}(N)$ is the canonical $q$-plane bundle over $B_{q}(N)$. Equivalently we may consider $\tilde{\rho}$ as a section of $\rho^{*}\left(E_{q}(N) \times \Delta^{k}\right)$. Let $\Sigma^{q}(\tau)$ denote $\tilde{\rho}^{-1}$ (zero section). 
Another description of $\Sigma^{q}(\tau)$ is the following: $\Sigma^{q}(\tau)=\left\{(x, t) \in \bar{W}_{q}(\sigma) \mid \xi_{t}^{q}(x)\right.$ lies in the span of $\left.\left\{\xi_{t}^{i}(x) \mid i>q\right\}\right\}$. By transversality $\Sigma^{q}(\tau)$ will be a closed $(k-q)$-manifold in the interior of $\bar{W}_{q}(\sigma)$. We call $\Sigma^{q}(\tau)$ the obstruction set because if it is empty we can make $\tau=(\sigma, \mathfrak{m}, \xi)(q-1)$-semiframed by projecting $\xi_{t}^{q}(x)$ to $\rho_{0}(x, t)$ as above and dividing it by its length.

If $h:\left(\Delta^{k}, \partial \Delta^{k}\right) \rightarrow\left(\mathscr{L}_{q}^{\Delta}(N), \mathscr{L}_{q-1}^{\Delta}(N)\right), h(t)=\left(f_{t}, \mathfrak{m}_{t}, \xi_{t}\right)$, is a simplicial map, then we can define $\bar{W}_{q}(f), \rho: \bar{W}_{q}(f) \rightarrow B_{q}(N) \times \Delta^{k}$, and $\Sigma^{q}(h)$ analogously. If we let $\tau=\tilde{j}_{q}^{\Delta} h$ and $\sigma_{t}=\tilde{j}^{q} f_{t}$, then we have $\bar{W}_{q}(f)=\bar{W}_{q}(\sigma), \rho_{h}=\rho_{\tau}$, and $\Sigma^{q}(h)=\Sigma^{q}(\tau)$.

Proposition 3.6. Suppose that $\tilde{\rho}: \bar{W}_{q}(\sigma) \rightarrow E_{q}(N) \times \Delta^{k}$ is transverse to the zero section $B_{q}(N) \times \Delta^{k} \rightarrow E_{q}(N) \times \Delta^{k}$. Then

(a) $\operatorname{dim} \Sigma^{q}(\tau)=k-q$ and

(b) the derivative of $\tilde{\rho}$ gives a bundle isomorphism

$$
\nu\left(\Sigma^{q}(\tau), W_{q}(\sigma)\right) \cong \rho^{*}\left(E_{q}(N) \times \Delta^{k}\right) \mid \Sigma^{q}(\tau) .
$$

If $q>k$, then $\Sigma^{q}(\tau)$ will be empty so we have

Proposition 3.7. The inclusion maps $\mathscr{L}_{q-1}^{\Delta}(N) \rightarrow \mathscr{L}_{q}^{\Delta}(N)$ and $\Gamma_{q-1}^{\Delta} \rightarrow \Gamma_{q}^{\Delta}$ are $(q-1)$-connected.

Now consider the following square which we call $\mathscr{S}_{q}$. The above Proposition (3.7) implies that this square is $(q-1)$-connected. We shall devote the next four sections to the proof of the following theorem.

Theorem 3.8. Let $q \geqslant 2$. Then the square $\mathscr{S}_{q}$ below is $(n+1)$-connected.

$$
\begin{array}{cccc} 
& \mathscr{L}_{q-1}^{\Delta}(N) & \stackrel{\tilde{j}_{q-1}^{\Delta}}{\rightarrow} & \Gamma_{q-1}^{\Delta} \\
\mathscr{S}_{q}: & \downarrow & & \downarrow \\
& \mathscr{L}_{q}^{\Delta}(N) & \stackrel{\tilde{j}_{q}^{\Delta}}{\rightarrow} & \Gamma_{q}^{\Delta}
\end{array}
$$

CoRollary 3.9. The map $\tilde{j}_{q}^{\Delta}: \mathscr{L}_{q}^{\Delta}(N) \rightarrow \Gamma_{q}^{\Delta}$ is n-connected for $q \geqslant 1$.

Proof. This holds for $q=n$ by Theorem 2.2 and Proposition 3.5(b). The corollary now follows from Theorem 3.8 by downward induction on $q$.

4. Movement of $\Sigma^{q}(h)$ into one index. Let $h:\left(\Delta^{k}, \partial \Delta^{k}\right) \rightarrow\left(\mathscr{L}_{q}^{\Delta}(N), \mathscr{L}_{q-1}^{\Delta}(N)\right)$, $h(t)=\left(f_{t}, \mathfrak{m}_{t}, \xi_{t}\right)$, be a simplicial map satisfying the transversality condition of Proposition 3.6. The purpose of this section is to show how $h$ can be deformed so that $\Sigma^{q}(h)$ moves by an isotopy into $A_{1}^{q}(f)$. We assume only $q \geqslant 1$.

LEMMA 4.1 (ISOTOPY LEMMA). Any isotopy of $\Sigma^{q}(h)$ in $W_{q}(f)$ can be realized by a deformation of $\xi_{t}$.

ProOF. We shall construct a deformation of $\xi_{t}$ which changes only the $q$ th vector $\xi_{t}^{q}$ in int $W_{q}(f)$. Since we are considering $\xi_{t}^{i}$ to be fixed for $i>q$ we have a fixed map $\rho: W_{q}(f) \rightarrow B_{q}(N) \times \Delta^{k}$ as discussed in $\S 3$ and $\xi^{q}$ is essentially given by a section $s$ of $E=\rho^{*}\left(E_{q}(N) \times \Delta^{k}\right)$ which is a $q$-plane bundle over $W=W_{q}(f)$. 

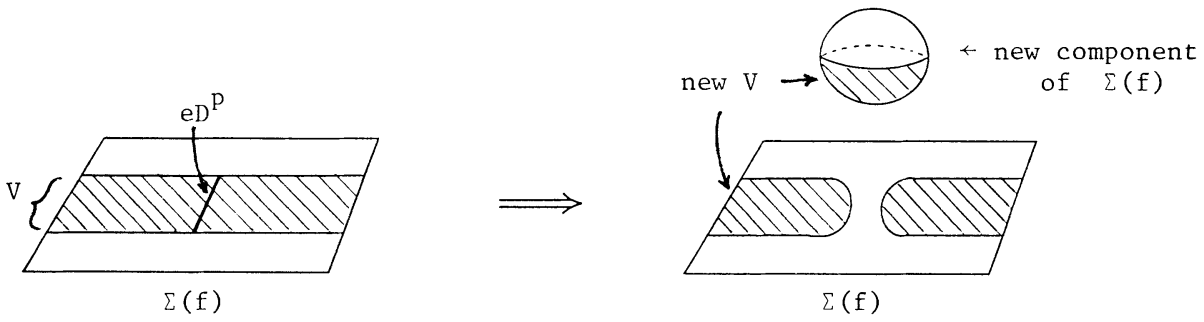

FIGURE D (removal of a $p$-cell from $V$ )

By the ambient isotopy lemma any isotopy of $\Sigma^{q}(h)$ in int $W$ is the restriction to $\Sigma^{q}(h)$ of a smooth family of diffeomorphisms $\phi_{u}: W \rightarrow W, u \in I$, where $\phi_{0}=1_{W}$ and $\phi_{u} \mid \partial W=1_{\partial W}$. Consider $\phi$ as a map $W \times I \rightarrow W$. Since $\phi^{*} E \cong E \times I$, we get a family of vector bundle isomorphisms $\tilde{\phi}_{u}: E \rightarrow E$ which cover the maps $\phi_{u}$. If we let $s_{u}=\tilde{\phi}_{u} s \phi_{u}^{-1}$, then $s_{u}^{-1}$ (zero section) $=\phi_{u} s^{-1}$ (zero section). Thus if $\xi^{q}$ is changed by the deformation $s_{u}$, then $\Sigma^{q}(h)$ changes by $\phi_{u}$.

Assume now that $n \geqslant 2$ and $i$ is a fixed integer such that $2 \leqslant i \leqslant n$. Let $V=A_{1}^{i}(f) \cup A_{2}^{i-1}(f)$. Then $V$ is a smooth $k$-manifold with $\partial V=A_{2}^{i-1}(f) \cup(V \cap$ $\left.\left(N \times \partial \Delta^{k}\right)\right)$ and the projections $A_{1}^{i}(f) \rightarrow \Delta^{k}, A_{2}^{i-1}(f) \rightarrow \Delta^{k}$ are immersions.

Lemma 4.2. Suppose that $p \leqslant n-2$ and $e:\left(D^{p}, \partial D^{p}\right) \rightarrow(V, \partial V)$ is a smooth embedding transverse to $\partial V$ so that the composition $D^{p} \rightarrow V \rightarrow \Delta^{k}$ is a topological embedding into int $\Delta^{k}$. Then there exists a deformation $\left(f_{t}^{u}, \mathfrak{m}_{t}^{u}, \xi_{t}^{u}\right)$ of $\left(f_{t}, \mathfrak{m}_{t}, \xi_{t}\right)$ with support in an arbitrarily small neighborhood $U$ of $e\left(D^{p}\right)$ so that $V$ changes by handle subtraction along $e\left(D^{p}\right)$ and by the addition of a new disjoint component diffeomorphic to $D^{k}$. Furthermore if $\Sigma^{q}(h)$ is disjoint from $U$, then $\Sigma^{q}\left(h^{u}\right)$ will remain disjoint from $U$ throughout the deformation. Figure $\mathrm{D}$ illustrates how the singular set $\Sigma(f)$ will change.

Proof. We first do the case $p=0$. In this case $e D^{p}=\left(x_{0}, t_{0}\right)$ is one point in the interior of $V$. By the normal form lemma for Morse points (A1.3) there is a neighborhood $T$ of $t_{0}$ in $\Delta^{k}$, a neighborhood $Y$ of 0 in $\mathbf{R}^{n}$, and a variable system of coordinates $\phi_{t}: Y \rightarrow N, t \in T$, so that $\phi_{t_{0}}(0)=x_{0}$ and $f_{t} \phi_{t}(y)=\sum_{j=1}^{i}-y_{j}^{2}+$ $\sum_{j=i+1}^{n} y^{2}+c(t)$ for all $(y, t) \in Y \times T$, where $c$ is a smooth map $T \rightarrow \mathbf{R}$. By a deformation of the metric $\mathfrak{m}_{t}$ and the partial framing $\xi_{t}$ we may assume that $\phi_{t}$ is an isometry and that $\xi_{t}^{j}\left(\phi_{t}(0)\right)=D \phi_{t}(0) \cdot e_{j}$ for $j \geqslant q+1$, where $e_{j}$ is the $j$ th unit vector in $\mathbf{R}^{n}$.

Since the functions $f_{t} \phi_{t}$ are the same up to a constant $c_{t}$ we may apply the "radiation" construction of $[\mathbf{I 2}, \S 4]$. Thus we may radiate the framed deformation described in Figure B in the proof of 1.5. This leads to the deformation indicated in Figure $\mathrm{E}$. The singular set changes as indicated in Figure $\mathrm{F}$ where the shaded area is a set of $A_{1}^{i-1}$ singularities bounded by $A_{2}^{i-1}$ singularities. The unshaded area is $V$. We have changed $V$ as desired. Note that the new singular set is diffeomorphic to the old one. 

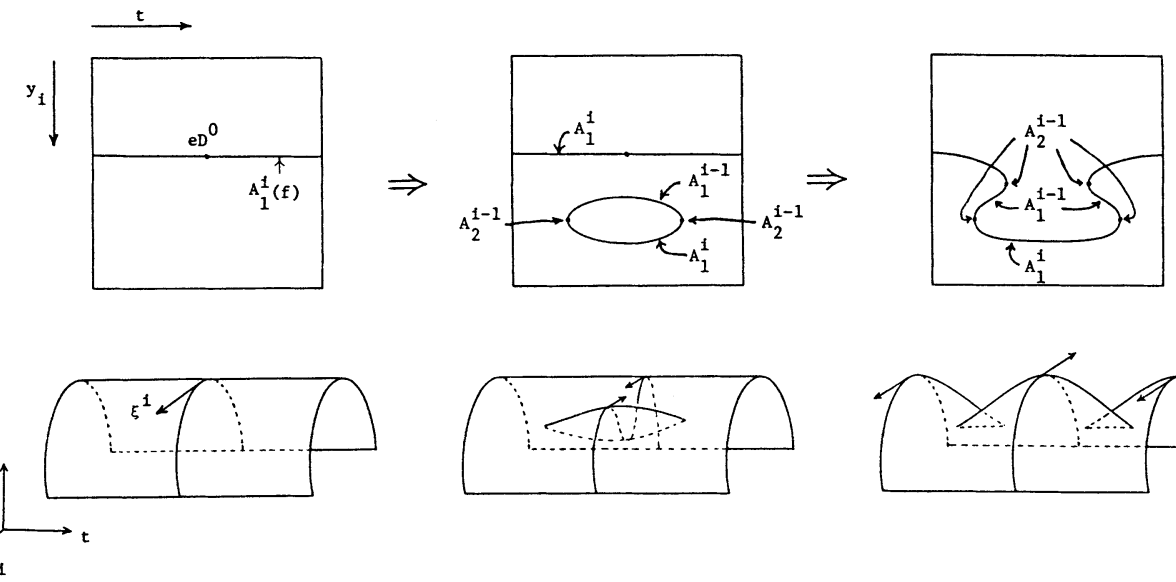

Figure E (removal of a 0-cell from $A_{1}^{i}(f)$ )
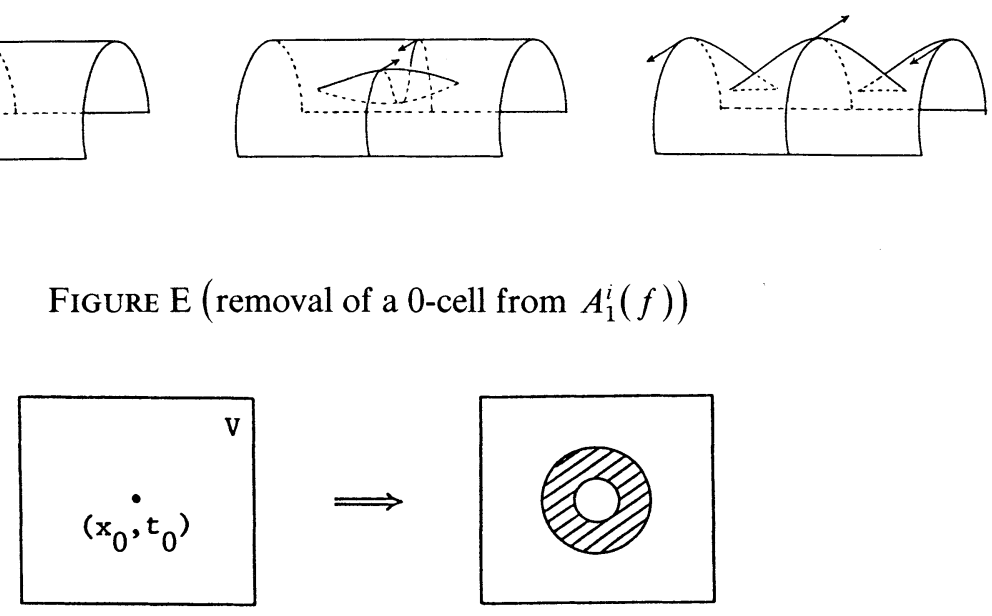

FIGURE F (removel of a 0 -cell from $V$ )
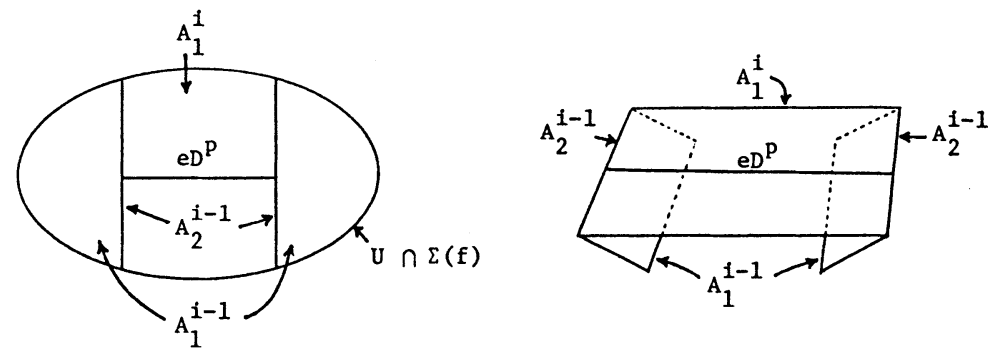

Figure G (a $p$-cell in $V)$

Now suppose that $p \geqslant 1$. Since $e D^{p}$ is contractible we may assume that our semiframed function is framed along $e D^{p}$. We may assume that $U$ is a small contractible neighborhood of $e D^{p}$. Then we have the singularities in $U$ shown in Figure G.

Consider a $(p-1)$-sphere $S^{p-1}$ of $A_{1}^{i-1}$ points which lies parallel to $e\left(\partial D^{p}\right)$ in $U$. Let $T$ be a tubular neighborhood of $S^{p-1}$ in $A_{1}^{i-1}(f) \cap U$ so that $\pi: T \rightarrow \Delta^{k}$ is an embedding. Let $Y$ be a neighborhood of 0 in $\mathbf{R}^{n}$ and let $\phi_{t}: Y \rightarrow N, t \in T$, be a smooth family of embeddings so that $\left(\phi_{t}(y), \pi(t)\right) \in U$ for all $(y, t) \in Y \times T$, $\left(\phi_{t}(0), \pi(t)\right)=t$ for all $t \in T$, and $f_{\pi(t)} \phi_{t}(y)=\sum_{j=1}^{i-1}-y_{j}^{2}+\sum_{j=i}^{n} y_{j}^{2}+c(t)$ for all $(y, t) \in Y \times T$. If we choose a product structure $T \cong S^{p-1} \times D^{k-p+1}$ and ignore the variable constant term $c(t)$, then $f_{\pi(t)} \phi_{t}$ can be thought of as $S^{p-1} \times($ a family of functions parameterized over $\left.D^{k-p+1}\right)$. Take the deformation of Figure $\mathrm{B}$ in the 
proof of 1.5 and radiate it in the $D^{k-p+1}$ direction. Then $S^{p-1} \times$ (the radiation deformation) is a generalized radiation deformation which performs the deformation of Figure B simultaneously at all points of $S^{p-1}$. Figure $\mathrm{H}$ illustrates the result. We have created a new component of $A_{1}^{i-2}(f)$ diffeomorphic to $S^{p-1} \times I \times S^{k-p}$.

Now take the $(p-1)$-sphere of $A_{2}^{i-2}$ points closest to $e\left(\partial D^{p}\right)$ as indicated in Figure $\mathrm{H}$. Since $p-1 \leqslant n-2$ we may apply the birth-death cancellation lemma (A2.3) to eliminate this sphere. This deformation can be performed in $U$. The result is indicated in Figure I.

The result is that $e D^{p}$ now forms the top of a $p$-lens which is null homotopic and the vector $\xi^{i}$ extends over the bottom of this $p$-lens in such a way that the conditions of the lens replacement lemma (A4.5) are satisfied. If we radiate the deformation given by the lens replacement lemma we get a deformation of $h$ which performs surgery on the $p$-lens and creates a disjoint $k$-lens as shown in Figure $\mathbf{J}$ when $p<k$.

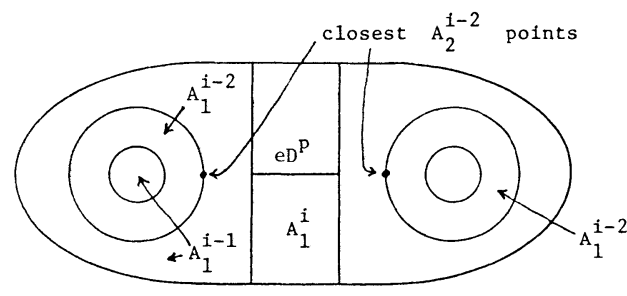

$(\mathrm{k}=\mathrm{p}+1)$

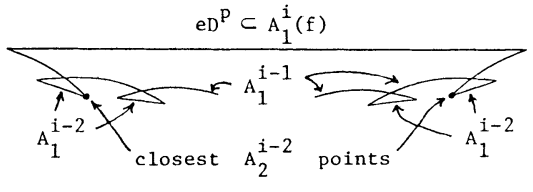

$(k=p)$ graphic

Figure H (create an $S^{p-1}$ of $A_{2}^{i-2}$ points)

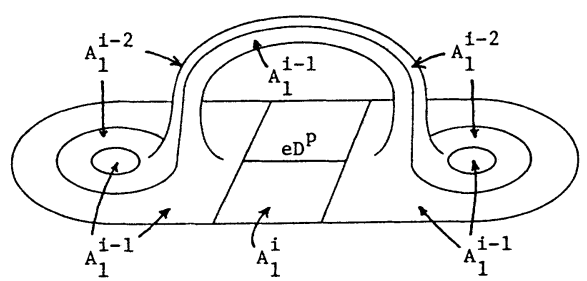

$(\mathrm{p}=\mathrm{k}-1)$

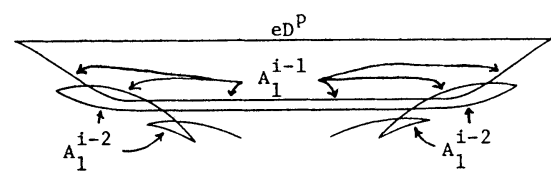

graphic $(p=k)$

FIgURE I (cancel the $S^{p-1}$ of $A_{2}^{i-2}$ points)

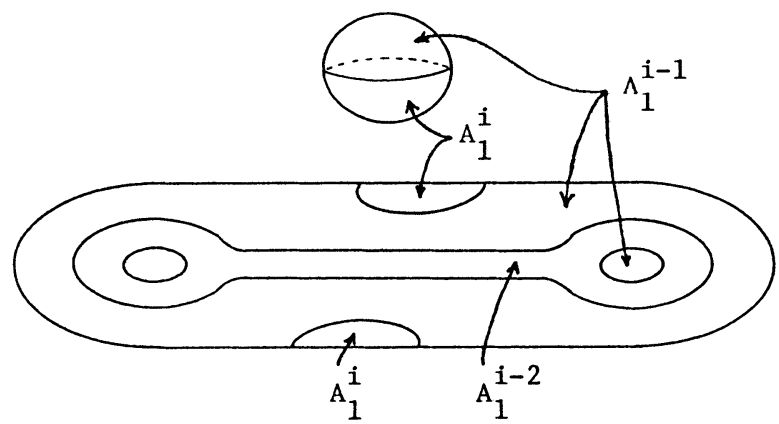

Figure $\mathbf{J}$ (eliminate the $p$-cell $e D^{p}$ ) 

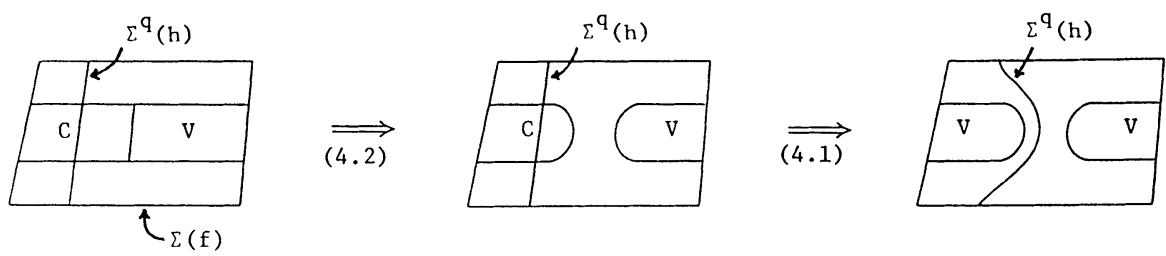

Figure K (slide $\Sigma^{q}(h)$ off of $\left.V\right)$

(If $p=k$, then we remove a $k$-lens and replace it with an identical $k$-lens so the picture does not change.) We have obtained the desired result. The singular set $\Sigma=\Sigma(f)$ has changed by the following cobordism.

(Figure H) $\quad \Sigma \rightarrow \Sigma \amalg S^{p-1} \times S^{k-p+1}$,

(Figure I) $\quad \Sigma \rightarrow \Sigma^{\prime}$ by surgery on a $(p-1)$-sphere,

(Figure J) $\quad \Sigma^{\prime} \rightarrow \Sigma^{\prime} \amalg S^{k}$,

$\Sigma^{\prime} \amalg S^{k} \rightarrow \Sigma \amalg S^{k}$ by surgery on a $p$-sphere.

The total cobordism is diffeomorphic to $\Sigma \times I \amalg D^{k+1}$.

THEOREM 4.3. Suppose that $k \leqslant n+q-2$. Then there is a q-semiframed deformation $h^{u}=\left(f_{t}^{u}, \mathrm{~m}_{t}^{u}, \xi_{t}^{u}\right)$ of $h=\left(f_{t}, \mathrm{~m}_{t}, \xi_{t}\right)$ with support in $\operatorname{int}\left(N \times \Delta^{k}\right)$ so that $\Sigma^{q}\left(h^{u}\right)$ changes by an isotopy as $u$ goes from 0 to 1 and $\Sigma^{q}\left(h^{1}\right) \subset A_{1}^{q}\left(f^{1}\right)$.

Proof. Let $i$ be an integer so that $q<i \leqslant n$ and suppose that $\Sigma^{q}(h)$ lies in $W_{q}^{i}(f)$, where $W_{q}^{i}=W_{q}-\bar{W}_{i+1}=A_{1}^{q} \cup A_{2}^{q} \cup \cdots \cup A_{2}^{i-1} \cup A_{1}^{i}$. Then we shall construct a deformation of $\left(f_{t}, \mathfrak{m}_{t}, \xi_{t}\right)$ with support in $\operatorname{int}\left(N \times \Delta^{k}\right)$ so that $\sum^{q}(h)$ moves by an isotopy into $W_{q}^{i-1}$. This will prove the theorem by downward induction on $i$.

Let $V=A_{1}^{i}(f) \cup A_{2}^{i-1}(f)$ as before and let $K=V \cap \Sigma^{q}(h)$. Then by transversality $K$ will be a compact $(k-q)$-manifold with boundary $\partial K=A_{2}^{i-1}(f) \cap \Sigma^{q}(h)$. Choose a cell decomposition of $K$ relative to $\partial K$ so that each closed cell maps homeomorphically onto a closed disk in $\Delta^{k}$. Let $C$ be a cell of minimal dimension. Then $C$ is a $p$-disk in $K$ with $\partial C \subset \partial K$. Choose a nonzero vector field for $\nu(K, V) \mid C$. This gives an embedding $e:\left(D^{p}, S^{p-1}\right) \rightarrow(V, \partial V)$ disjoint from $K$ which is parallel to $C$. Since $p \leqslant \operatorname{dim} K=k-q \leqslant n-2$ we may apply Lemma 4.2 with $U$ disjoint from $\Sigma^{q}(h)$. We can then "slide" the cell $C$ off of $V$ as indicated in Figure $\mathrm{K}$ by the isotopy lemma (4.1). This changes $K$ by handle removal along $C$ so by repeating this process $K$ will disappear.

5. Surgery on $\Sigma^{q}(\tau)$. Let $\tau: \Delta^{k} \rightarrow \Gamma_{q}$ be a $k$-simplex in $\Gamma_{q}^{\Delta}$ satisfying the transversality condition of Proposition 3.6. Then we shall show that the obstruction set $\sum^{q}(\tau)$ can be modified by any "two stage admissible surgery" provided that $n+1 \geqslant k \geqslant q-1 \geqslant 1$. We begin with the definition of "admissible surgery" using the notation of $[\mathbf{I} 2, \S 7]$.

Let $L, Y$ be smooth manifolds and let $p: Y \rightarrow L$ be a smooth bundle map. Let $X$ be a nonempty closed submanifold of $Y$ so that $X$ is also a smooth subbundle of $Y$ over $L$ and suppose that $\operatorname{dim} Y-\operatorname{dim} X \geqslant 1$. Let $f: L \rightarrow Y$ be a smooth section of 
$p$ which is transverse to $X$ and let $M=f^{-1}(X)$. Then $M$ is a smooth manifold properly embedded in $L$ and $\nu(M, L) \cong(f \mid M)^{*} \nu(X, Y)$. If $M$ is empty we add the extra condition that $l-\operatorname{dim} Y+\operatorname{dim} X \geqslant-1$, where $l=\operatorname{dim} L$.

DEFINITION 5.1. A (one stage) admissible surgery on $M$ consists of a quadruple $(\theta, c, \eta, \gamma)$, where

$\theta: S^{i} \rightarrow$ int $M$ is a framed embedding,

$c: D^{i+1} \rightarrow X$ is a contraction of $f \theta: S^{i} \rightarrow X$,

$\eta$ is a framing of the trivial bundle $c^{*} \nu(X, Y)$,

$\gamma: D^{i+1} \rightarrow L$ is a framed null-isotopy of $\theta\left(S^{i}\right)$ in $L$ which is homotopic rel $S^{i}$ to pc. By framed null isotopy we mean:

(1) $\gamma$ is a framed embedding.

(2) The first $m-i$ vectors in the framing of $\gamma\left(D^{i+1}\right) \mid S^{i}$ coincide with the given framing of $\theta\left(S^{i}\right)$ in $M$.

(3) The last $l-m-1$ vectors in the framing of $\gamma\left(D^{i+1}\right) \mid S^{i}$ together with the inward normal vector of $\gamma\left(S^{i}\right)$ in $\gamma\left(D^{i+1}\right)$ form a framing of $\nu(M, L) \mid \theta\left(S^{i}\right)$ which agrees with $\eta \mid S^{i}$ under the isomorphism

$$
\nu(M, L)\left|\theta\left(S^{i}\right) \cong \theta^{*} \nu(M, L) \cong \theta^{*} f * \nu(X, Y) \cong c^{*} \nu(X, Y)\right| S^{i}
$$

An isotopy of $(\theta, c, \eta, \gamma)$ is defined to be a simultaneous smooth deformation of $\theta, c$, $\eta, \gamma$.

REMARKS. (a) Since $p c \simeq \gamma \operatorname{rel} S^{i}$ and $X$ is a bundle over $L$ we may deform $c$ rel $S^{i}$ so that $p c=\gamma$.

(b) We allow the case $i=-1, S^{-1}=\varnothing, D^{0}=*$.

Suppose that $h: L \times I \rightarrow Y$ is a deformation of $f$, i.e. $h(-, 0)=f$ and $p h$ : $L \times I \rightarrow L$ is the projection map. Suppose also that $h$ is transverse to $X$ and that $h^{-1}(X)=W$ is an elementary cobordism of $M$ in the sense that the projection map $L \times I \rightarrow I$ when restricted to $W$ gives a Morse function $g: W \rightarrow I$ with exactly one critical point which has index, say, $i+1$. Let $e^{i+1} \subset W$ be the core of the $i+1$ handle corresponding to this critical point and let $\alpha: D^{i+1} \rightarrow e^{i+1}$ be a diffeomorphism.

Suppose that $1 / 2 \in I$ is the unique critical value of $g: W \rightarrow I$. Then the disk $e^{i+1} \cap g^{-1}[1 / 2-\varepsilon, 1 / 2]$ is isotopic fixing its boundary to an $(i+1)$-disk in $L \times$ $(1 / 2-\varepsilon)$ for $\varepsilon>0$ sufficiently small. Since $e^{i+1} \cap g^{-1}[0,1 / 2-\varepsilon]$ is an isotopy of an embedded $i$-sphere in $L$, we can use the ambiant isotopy lemma to show that $\alpha$ : $D^{i+1} \rightarrow W$ is isotopic rel $S^{i}$ to an embedding $\gamma: D^{i+1} \rightarrow L$.

Choose framings $\zeta$ and $\eta$ of $\nu\left(e^{i+1}, W\right)$ and $\nu(W, L \times I) \mid e^{i+1}$ respectively. Then $\zeta$ gives a framing of $e^{i+1}=\alpha\left(D^{i+1}\right)$ in $W$ and $(\zeta, \eta)$ gives a framing of $\alpha\left(D^{i+1}\right)$ in $L \times I$. The isotopy $\alpha \simeq \gamma$ gives a framing of $\gamma$ in $L$ by deleting the last vector of $\eta$ (the isotopy direction).

Let $\theta=\alpha \mid S^{i}: S^{i} \rightarrow M$ with the framing $\zeta \mid S^{i}$. Let $c=h \alpha: D^{i+1} \rightarrow X$. Consider $\eta$ as a framing on

$$
c^{*} \nu(X, Y)=(h \alpha)^{*} \nu(X, Y)=\alpha^{*} \nu(W, L \times I)=\nu(W, L \times I) \mid e^{i+1}
$$


Then $(\theta, c, \eta, \gamma)$ is an admissible surgery on $M$. We say that the deformation $h$ of $f$ is a realization of the admissible surgery $(\theta, c, \eta, \gamma)$.

Note that the framings $\zeta$ and $\eta$ were chosen arbitrarily. Thus $(\theta, c, \eta, \gamma)$ is not uniquely determined by $h$. (Since there are only two choices for $\eta$ and $\zeta$ up to homotopy and $\theta, c, \gamma$ are unique up to framing there are at most four distinct isotopy classes of admissible surgeries for each $h$, but this is not important.)

The important uniqueness property of realizations is the following. A realization of a surgery on an $i$-sphere produces "half" of a realization of a surgery on an $(i+1)$-sphere. Thus if $f^{u}, u \in[0,1]$, and $f^{u}, u \in[-1,0]$, are two realizations of the same admissible surgery up to isotopy then we get another admissible surgery on $M \times[-1,1]$ with $\theta^{\prime}=e^{i+1} \cup\left(e^{i+1}\right)^{\prime}=$ the union of the two handle cores. A realization of this higher admissible surgery produces an isotopy between $f^{1}$ and $f^{-1}$. Thus any realizability result for admissible surgeries also gives a uniqueness up to isotopy result for realizations of the same surgery.

In $[\mathbf{I} 2,7.6]$ we explained the following more or less trivial realizability result for admissible surgeries.

THEOREM 5.2. Suppose that

(a) $i+2<\operatorname{dim} Y$ and

(b) each component of each fiber of Y over L has $\pi_{i+1}=0$.

Then any admissible surgery on an $i$-sphere in $M$ can be realized up to isotopy by a deformation of $f$ with support in the interior of $L$.

We now return to $q$-semiframed sections. Let $\tau: \Delta^{k} \rightarrow \Gamma_{q}$ be a $k$-simplex of $\Gamma_{q}^{\Delta}$, where $\tau(t)=\left(\sigma_{t}, \mathrm{~m}_{t}, \xi_{t}\right)$, and let $\Sigma^{q}(\tau) \subset \bar{W}_{q}(\sigma) \subset \Sigma_{q}(\sigma)$ be the obstruction set defined in $\S 3$. We assume the transversality condition of 3.6 so $\Sigma^{q}(\tau)$ will be a smooth $(k-q)$-manifold. We would like to show that (under certain conditions) $\Sigma^{q}(\tau)$ can be changed by surgery by a deformation of $\tau$. We begin with the following preparation lemma analogous to Theorem 4.3.

Lemma 5.3. Suppose that $\sum^{q}(\tau) \cap\left(N \times \partial \Delta^{k}\right)$ lies in $A_{1}^{q}(\sigma)$. Then there exists $a$ deformation $\tau^{u}$ of $\tau$ with support in $\operatorname{int}\left(N \times \Delta^{k}\right)$ so that $W_{q}(\sigma)$ and $\Sigma^{q}(\tau)$ remain fixed as sets but $\Sigma^{q}\left(\tau^{1}\right)=\Sigma^{q}(\tau) \subset A_{1}^{q}\left(\sigma^{1}\right)$. Furthermore

(1) $A_{1}^{q}\left(\sigma^{u}\right) \supset A_{1}^{q}(\sigma)$ for all $u \in I$.

(2) Given any compact set $K$ in int $\Delta^{k}$ we can choose $\tau^{u}$ so that $W_{q}\left(\sigma^{1}\right)=A_{1}^{q}\left(\sigma^{1}\right)$ in $N \times K$.

Proof. Suppose for a moment that there are no boundary restrictions (i.e. suppose that we are allowed to deform $\tau$ on all of $N \times \Delta^{k}$ ). Then we can move $\sigma_{t}$ on the entire set $W_{q}(\sigma)$ so that it moves into $A_{1}^{q}(\sigma)$ and condition (1) is satisfied. To see this recall the space $C_{q}(n)$ of Proposition 2.10. This is a space of pairs $(p, \zeta)$ where $p \in W_{q}(n)$ and $\zeta=\left(\xi^{q+1}, \xi^{q+2}, \ldots\right) . C_{q}(n)$ is an $O(n)$-space and the projection to the first factor $C_{q}(n) \rightarrow W_{q}(n)$ is $O(n)$-equivariant. Note that each point of $A_{1}^{q}(n)$ $\subset W_{q}(n)$ has a unique inverse image point in $C_{q}(n)$ so we get a map $A_{1}^{q}(n) \rightarrow C_{q}(n)$. 
Let $T N$ denote the tangent bundle of $N$. Then the variable metric $\mathrm{m}_{t}$ gives a reduction of the structure groups of $T N \times \Delta^{k}, H(N) \times \Delta^{k}$, etc. to $O(n)$ so we get the following bundle maps over $N \times \Delta^{k}$ :

$$
\begin{aligned}
& C_{q}(N) \times \Delta^{k} \\
& \begin{array}{ccccc}
A_{1}^{q}(N) \times \Delta^{k} & \underset{c}{\nearrow} & W_{q}(N) \times \Delta^{k} & \underset{c}{\rightarrow} & H(N) \times \Delta^{k}
\end{array}
\end{aligned}
$$

The section $\sigma_{t}$ of $H(N) \times \Delta^{k}$ restricts to a section of $W_{q}(N) \times \Delta^{k}$ over $W_{q}(\sigma)$ and $\zeta_{t}\left(\xi_{t}^{q+1}, \xi_{t}^{q+2}, \ldots\right)$ gives a lifting of this section to $C_{q}(N) \times \Delta^{k}$.

According to Proposition 2.10, $C_{q}(n) \simeq O(n) /(O(q) \times O(n-q))$. Since the composition $A_{1}^{q}(n) \rightarrow C_{q}(n) \rightarrow O(n) /(O(q) \times O(n-q))$ is a homotopy equivalence we see that $A_{1}^{q}(n) \simeq C_{q}(n)$. This implies that the section $\left(\sigma_{t}, \xi_{t}\right)$ of $C_{q}(N) \times \Delta^{k}$ over $W_{q}(\sigma)$ can be deformed into $A_{1}^{q}(N) \times \Delta^{k}$. This deformation can be chosen to have compact support since $\left(\sigma_{t}, \xi_{t}\right)^{-1}\left(C_{q}(N)-A_{1}^{q}(N)\right) \times \Delta^{k}=\bar{W}_{q+1}(\sigma)$ is compact. The normal bundle of $W_{q}(N) \times \Delta^{k}$ in $H(N) \times \Delta^{k}$ is fiberwise trivial so the deformation of $\sigma_{t}$ can be extended to all of $N \times \Delta^{k}$ so that it has compact support in an arbitrarily small neighborhood of $\bar{W}_{q+1}(\sigma)$. Note that $W_{q}(\sigma)$ remains fixed throughout the deformation.

Let $\sigma_{t}^{u}, \xi_{t}^{u}, u \in I$, denote the deformations of $\sigma_{t}, \xi_{t}^{q+1}, \xi_{t}^{q+2}, \ldots$ just obtained. Then $\bar{W}_{q}\left(\sigma^{u}\right)=\bar{W}_{q}(\sigma)$ for all $u$ so we have a deformation $\rho^{u}$ of $\rho: \bar{W}_{q}(\sigma) \rightarrow B_{q}(N)$ $\times \Delta^{k}$. By the covering homotopy property there exists a family of sections $g^{u}$ of (the principal tangent bundle of $N) \times \Delta^{k}$ over $\bar{W}_{q}(\sigma)$ so that $g^{0}$ is identically 1 and $\rho^{u}=g^{u} \rho$ for all $u$. Define the deformations $\left(\xi_{t}^{l}\right)^{u}, \ldots,\left(\xi_{t}^{q}\right)^{u}$ by $\left(\xi_{t}^{i}\right)^{u}=g^{u} \xi_{t}^{i}$. Then $\Sigma^{q}\left(\tau^{u}\right)=\Sigma^{q}(\tau)$ for all $u$.

Let us return to the proof of Lemma 5.3. If $\Sigma^{q}(\tau) \subset A_{1}^{q}(\sigma)$ over $\partial \Delta^{k}$, then there is a neighborhood $U$ of $\partial \Delta^{k}$ in $\Delta^{k}$ so that $\Sigma^{q}(\tau) \subset A_{1}^{q}(\sigma)$ over $U$. Given any compact $K \subset$ int $\Delta^{k}$ let $\phi: \Delta^{k} \rightarrow I$ be a smooth map so that $\left(\Delta^{k}-U\right) \cup K$ is contained in $\phi^{-1}(1)$ and $\partial \Delta^{k} \subset \phi^{-1}(0)$. Let $\tau_{t}^{u}=\left(\sigma_{t}^{u}, \mathfrak{m}_{t}, \xi_{t}^{u}\right)$ be the deformation obtained above. Then $\tau_{t}^{u \phi(t)}$ is the desired deformation.

Let $\beta: A_{1}^{q}(N) \times \Delta^{k} \rightarrow B_{q}(N) \times \Delta^{k}$ be the map which sends $(g, t)$ to $(b, t)$, where $b$ is the negative eigenspace of $D^{2} g$ (made into a tangent $q$-plane by the metric $\mathfrak{m}_{t}$ ). Then $\beta$ is a homotopy equivalence since $A_{1}^{q}(n) \simeq O(n) /(O(q) \times O(n-q))$.

Definition 5.4. Suppose that $\tau=\left(\sigma_{t}, \mathrm{~m}_{t}, \xi_{t}\right)$ is a $k$-simplex of $\Gamma_{q}^{\Delta}$ and $\Sigma^{q}(\tau) \subset$ $A_{1}^{q}(\sigma)$. Then we define a two-stage admissible surgery on $\Sigma^{q}(\tau)$ to be a 5 -tuple $(\theta, c, \eta, \zeta, \gamma)$ where

$\theta: S^{i} \rightarrow \sum^{q}(\tau)$ is a framed embedding,

$c: D^{i+1} \rightarrow A_{1}^{q}(N) \times \Delta^{k}$ is a smooth contraction of $\bar{\sigma} \theta\left(\bar{\sigma}: A_{1}^{q}(\sigma) \rightarrow A_{1}^{q}(N) \times \Delta^{k}\right.$ being the restriction of $\sigma$ ),

$\eta$ is a framing of the trivial $n$-plane bundle $c^{*} \nu\left(A_{1}^{q}(N) \times \Delta^{k}, H(N) \times \Delta^{k}\right)$,

$\zeta$ is a framing of the trivial $q$-plane bundle $c^{*} \beta^{*}\left(E_{q}(N) \times \Delta^{k}\right)$,

$\gamma: D^{i+1} \rightarrow N \times \Delta^{k}$ is a framed null-isotopy of $\theta$ which is homotopic rel $S^{i}$ to $p \sigma c$. 
The framing of $\theta\left(S^{i}\right)$ in $N \times \Delta^{k}$ is given in the obvious way by $\theta, \eta, \zeta$. The inward normal of $\gamma\left(S^{i}\right)=\theta\left(S^{i}\right)$ in $\gamma\left(D^{i+1}\right)$ is the last $\zeta$ vector (which, by the way, is tangent to $\left.A_{1}^{q}(\sigma)\right)$.

Suppose that $\tau^{u}=\left(\sigma_{t}^{u}, \mathfrak{m}_{t}^{u}, \xi_{t}^{u}\right)$ is a deformation of $\tau$ so that $\Sigma^{q}\left(\tau^{u}\right) \subset A_{1}^{q}\left(\sigma^{u}\right) \subset$ $N \times \Delta^{k} \times I$ and $\Sigma^{q}\left(\tau^{u}\right)$ is an elementary cobordism of $\Sigma^{q}(\tau)$ in the sense discussed above. Then we get a two-stage admissible surgery $(\theta, c, \eta, \zeta, \gamma)$ as follows. Since $\Sigma^{q}\left(\tau^{u}\right)$ is an elementary cobordism there is an embedding $\alpha:\left(D^{i+1}, S^{i}\right) \rightarrow$ ( $\left.\Sigma^{q}\left(\tau^{u}\right), \Sigma^{q}(\tau)\right)$ which is the core of the handle being attached to $\Sigma^{q}(\tau)$. Since $\Sigma^{q}\left(\tau^{u}\right) \subset A_{1}^{q}\left(\tau^{u}\right)$ the section $\sigma^{u}$ of $H(N) \times \Delta^{k} \times I$ induces a map $\bar{\sigma}^{u}: \Sigma^{q}\left(\tau^{u}\right) \rightarrow$ $A_{1}^{q}(N) \times \Delta^{k}$. Choose framings for $\nu\left(\alpha\left(D^{i+1}\right), \Sigma^{q}\left(\tau^{u}\right)\right), \alpha^{*} \nu\left(\Sigma^{q}\left(\tau^{u}\right), A_{1}^{q}\left(\sigma^{u}\right)\right)$, and $\alpha^{*} \nu\left(A_{1}^{q}\left(\sigma^{u}\right), N \times \Delta^{k} \times I\right)$. Then:

(1) $\theta=\alpha \mid S^{i}: S^{i} \rightarrow \Sigma^{q}(\tau)$ becomes a framed embedding.

(2) $c=\bar{\sigma}^{u} \alpha: D^{i+1} \rightarrow A_{1}^{q}(N) \times \Delta^{k}$ is a contraction of $\bar{\sigma} \theta$.

(3) We get a framing $\eta$ of

$$
c^{*} \nu\left(A_{1}^{q}(N) \times \Delta^{k}, H(N) \times \Delta^{k}\right) \cong \alpha^{*} \nu\left(A_{1}^{q}(\sigma), N \times \Delta^{k} \times I\right) .
$$

(4) We get a framing $\zeta$ of $c^{*} \beta^{*}\left(E_{q}(N) \times \Delta^{k}\right) \cong \alpha^{*} \nu\left(\Sigma^{q}\left(\tau^{u}\right), A_{1}^{q}\left(\sigma^{u}\right)\right)$.

(5) By isotopy extension, $\alpha: D^{i+1} \rightarrow \Sigma^{q}\left(\tau^{u}\right) \subset N \times \Delta^{k} \times I$ is isotopic rel $S^{i}$ to an embedding $\gamma: D^{i+1} \rightarrow N \times \Delta^{k}$ where the inward normal of $\gamma\left(S^{i}\right)$ in $\gamma\left(D^{i+1}\right)$ is the last $\eta$ vector.

We say that $\tau^{u}$ is a realization of the surgery $(\theta, c, \eta, \zeta, \gamma)$. As before the uniqueness of realizations up to isotopy is equivalent to the realizability of a surgery in one higher dimension.

THEOREM 5.5. Suppose that $n+1 \geqslant k \geqslant q-1 \geqslant 1, \tau$ is a $k$-simplex of $\Gamma_{q}^{\Delta}$, and $\Sigma^{q}(\tau) \subset A_{1}^{q}(\sigma)$. Then any two-stage admissible surgery on $\Sigma^{q}(\tau)$ can be realized up to isotopy by a deformation of $\tau$ with support in the interior of $N \times \Delta^{k}$.

Proof. We will construct an associated one-stage surgery which can be realized by Theorem 5.2. It will be clear that any realization of the one-stage surgery leads to a realization of the two-stage surgery.

Let $(\theta, c, \eta, \zeta, \gamma)$ be a two-stage admissible surgery on $\Sigma^{q}(\tau)$. Then we may assume that $p c=\gamma$ and by Lemma 5.3 we may assume that $W_{q}(\sigma)=A_{1}^{q}(\sigma)$ near $\gamma\left(D^{i+1}\right)$. The associated one-stage surgery $\left(\theta^{\prime}, c^{\prime}, \eta^{\prime}, \gamma^{\prime}\right)$ is defined as follows using the notation of Definition 5.1.

(1) $L$ is a closed contractible neighborhood of $\gamma\left(D^{i+1}\right)$ in $N \times \Delta^{k}$ so that $W_{q}(\sigma)=A_{1}^{q}(\sigma)$ in $L$.

(2) $\bar{c}: L \rightarrow A_{1}^{q}(N) \times \Delta^{k} \mid L$ is a smooth section so that $\bar{c} \gamma=c$.

(3) $Y$ is the fiber product of $\left(H(N)-\bar{W}_{q+1}(N)\right) \times \Delta^{k} \mid L$ and $\bar{c}^{*} \beta^{*}\left(E_{q}(N) \times \Delta^{k}\right)$ over $L$. Thus $Y \rightarrow L$ has fiber $\left(H(n)-\bar{W}_{q+1}(n)\right) \times \mathbf{R}^{q}$.

(4) $X$ is the subbundle of $Y$ with fiber $\left(\bar{W}_{q}(n)-\bar{W}_{q+1}(n)\right) \times 0=\left(A_{1}^{q}(n)-\right.$ $\left.A_{2}^{q-1}(n)\right) \times 0$.

(5) $f: L \rightarrow Y$ is given by $f=(\sigma, \bar{\xi})$, where $\bar{\xi}$ is any smooth section of the $q$-plane bundle $\bar{c}^{*} \beta^{*}\left(E_{q}(N) \times \Delta^{k}\right)$ over $L$ which agrees with $\xi_{t}^{q}$ on $\bar{W}_{q}(\sigma) \cap L$.

(6) $\theta^{\prime}=\theta: S^{i} \rightarrow M=\sum^{q}(\tau)$. 
(7) $c^{\prime}=(c, 0): D^{i+1} \rightarrow X$.

(8) $\eta^{\prime}=(\eta, \zeta)$.

(9) $\gamma^{\prime}=\gamma$.

We now verify the conditions of Theorem 5.2.

(a) Is $i+3 \leqslant \operatorname{dim} Y-\operatorname{dim} X$ ? Yes, because $\operatorname{dim} Y-\operatorname{dim} X=n+q$ and $i \leqslant k$ $-q \leqslant n+1-q \leqslant n-1$ so $i+3 \leqslant n+2 \leqslant n+q$.

(b) Do the fibers of $Y$ over $L$ have $\pi_{i+1}=0$ ? Yes, because

$$
\operatorname{fiber}(Y \rightarrow L) \simeq H(n)-\bar{W}_{q+1}(n) \simeq S^{n}\left(\Sigma(n)-\bar{W}_{q+1}(n)\right)
$$

is $n$-connected since $\Sigma(n)-\bar{W}_{q+1}(n)$ is connected and $i+1 \leqslant n$.

By Theorem 5.2 there is a deformation $\left(\sigma^{u}, \bar{\xi}^{u}\right)$ of $f=(\sigma, \bar{\xi})$ with compact support in the interior of $L$ which realizes the admissible surgery $\left(\theta^{\prime}, c^{\prime}, \eta^{\prime}, \gamma^{\prime}\right)$. Let $\tau^{u}=\left(\sigma_{t}^{u}, m_{t}, \xi_{t}^{u}\right)$ be the deformation of $\tau$ with support in $L$ given by $\sigma_{t}^{u}=\sigma^{u}$ and $\left(\xi_{t}^{q}\right)^{u}=\bar{\xi}^{u}$ on $L$. (The vectors $\left(\xi_{t}^{j}\right)^{u}$ can be chosen arbitrarily when $j<q$.) It follows from the definitions that if $\left(\sigma^{u}, \bar{\xi}^{u}\right)$ is a realization of $\left(\theta^{\prime}, c^{\prime}, \eta^{\prime}, \gamma^{\prime}\right)$, then $\tau^{u}$ is a realization of $(\theta, c, \eta, \zeta, \gamma)$ up to isotopy.

6. Surgery on $\Sigma^{q}(h)$. Let $h:\left(\Delta^{k}, \partial \Delta^{k}\right) \rightarrow\left(\mathscr{L}_{q}(N), \mathscr{L}_{q-1}(N)\right), h(t)=\left(f_{t}, \mathfrak{m}_{t}, \xi_{t}\right)$, be a simplicial map satisfying the transversality condition of Proposition 3.6 and the condition obtained in $\S 4$ that $\Sigma^{q}(h) \subset A_{1}^{q}(f)$. We define a two-stage admissible surgery on $\Sigma^{q}(h)$ to be one on $\Sigma^{q}(\tau)$, where $\tau=\tilde{j}_{q}^{\Delta} h$. A realization of such a surgery up to isotopy is defined to be a deformation $h^{u}$ of $h$ so that $\tau^{u}=\tilde{j}_{q}^{\Delta} h^{u}$ realizes the same surgery.

THEOREM 6.1. Suppose that $n \geqslant k \geqslant q-1 \geqslant 1$ and $\Sigma^{q}(h) \subset A_{1}^{q}(f)$. Then any two-stage admissible surgery $(\theta, c, \eta, \zeta, \gamma)$ on $\Sigma^{q}(h)$ can be realized up to isotopy by $a$ deformation of $h$ with support in the interior of $N \times \Delta^{k}$.

Proof. The proof is in two parts. First we show that the two-stage surgery $(\theta, c, \eta, \zeta, \gamma)$ can be realized assuming that $\gamma$ is in "standard form." Then we show that $\gamma$ can always be moved into standard form by a deformation of $h$ which changes $\Sigma^{q}(h)$ only by isotopy. As before we assume that $p c=\gamma$.

We say that $\gamma$ is in standard form if it satisfies the following.

(1) The composition $D^{i+1} \stackrel{\gamma}{\rightarrow} N \times \Delta^{k} \rightarrow \Delta^{k}$ is an embedding.

(2) The last $n$ vectors in the framing of $\gamma\left(D^{i+1}\right)$ in $N \times \Delta^{k}$ are parallel to $N$ and agree with the framing $\eta$ of $T N \times \Delta^{k} \mid \gamma\left(D^{i+1}\right) \cong c^{*} \nu\left(A_{1}^{q}(N) \times \Delta^{k}, H(N) \times \Delta^{k}\right)$.

Note that condition (2) holds along $\gamma\left(S^{i}\right)$ as part of the definition of a two-stage admissible surgery.

LEMMA 6.2. If $\gamma$ is in standard form (and $n \geqslant k \geqslant q-1 \geqslant 1$ ), then the two-stage surgery $(\theta, c, \eta, \zeta, \gamma)$ can be realized up to isotopy by a deformation of $h$ with support in an arbitrarily small neighborhood of $\gamma\left(D^{i+1}\right)$ in $N \times \Delta^{k}$.

Proof. Note first that $i+1 \leqslant k-q+1 \leqslant n-q+1<n=$ the codimension of $\Sigma(f)$ in $N \times \Delta^{k}$. Consequently we may assume using transversality that $\gamma\left(D^{i+1}\right)$ is disjoint from the singular set $\Sigma(f)$ except near $\gamma\left(S^{i}\right)$ where it meets $A_{1}^{q}(f)$. Since our deformation will have support in a small neighborhood of $\gamma\left(D^{i+1}\right)$ we may 
restrict our attention to such a neighborhood. By condition (1) we have the following situation.

Let $\bar{D}^{i+1}$ be an $(i+1)$-disk of radius $1+\varepsilon$, and let $D_{\varepsilon}^{k-i-1}, D_{\varepsilon}^{n}$ be disks of radius $\varepsilon$ of the indicated dimensions for some $\varepsilon>0$. Let $K=\bar{D}^{i+1} \times D_{\varepsilon}^{k-i-1}$. Then we have:

(a) $f_{t}: D_{\varepsilon}^{n} \rightarrow \mathbf{R}, t \in K$, a smooth family of smooth maps with only $A_{1}^{q}$-singularities. Thus $\Sigma\left(f_{t}\right)=A_{1}^{q}\left(f_{t}\right)$.

(b) We may assume that $\mathfrak{m}_{t}$ is the standard metric on $D^{n}$ for all $t \in K$.

(c) We have the map $\beta: A_{1}^{q}\left(f_{t}\right) \rightarrow B_{q}(n)=O(n) /(O(q) \times O(n-q))=($ the space of $q$-planes in $\mathbf{R}^{n}$ ) given by $\beta(x)=$ the negative eigenspace of $D^{2} f_{t}$ at $x$.

(d) $\xi$ assigns to each $x \in A_{1}^{q}(f)$ a vector in $\beta(x)$. (Since $\xi^{1}, \ldots, \xi^{q-1}$ are irrelevant we will assume that $\xi$ consists of the single vector $\xi^{q}$.) Thus $\xi$ is a lifting of $\beta$ to $E_{q}(n)=$ the canonical $q$-plane bundle over $B_{q}(n)$.

The two-stage surgery $(\theta, c, \eta, \zeta, \gamma)$ gives us the following.

(e) $\theta: S^{i} \rightarrow \Sigma^{q}(h)=\xi^{-1}(0)$. We may assume that $\theta(t)=(0, t) \in D_{\varepsilon}^{n} \times \bar{D}^{i+1}$ for all $t \in S^{i} \times 0 \subset \bar{D}^{i+1} \times D_{\varepsilon}^{k-i-1}=K$.

(f) $c: D^{i+1} \rightarrow B_{q}(n)$ is a contraction of $\beta \theta$. By choosing a lifting of $c$ to $O(n)$ we can rotate the disks $D_{\varepsilon}^{n}$ and assume that $c$ and $\beta$ are constant maps with image point $\mathbf{R}^{q} \subset \mathbf{R}^{n}$.

(g) $\zeta$ gives a framing of what is now the fixed plane $\mathbf{R}^{q}$ for each point in $D^{i+1}$. By rotating $D_{\varepsilon}^{n}$ again we may assume that $\zeta$ is the standard framing of $\mathbf{R}^{q}$.

(h) Since $\xi: A_{1}^{q}(f) \rightarrow \mathbf{R}^{q}$ is transverse to zero we get an induced framing $\zeta$ of $\nu\left(\Sigma^{q}(h), A_{1}^{q}(f)\right)$.

(i) The $k-q-i$ vectors of the framing of $\theta\left(S^{i}\right)$ in $\Sigma^{q}(h)$ and the first $q-1$ vectors of $\zeta$ form a framing of $\nu\left(D^{i+1} \times 0, \bar{D}^{i+1} \times D_{\varepsilon}^{k-i-1}\right)$. By rotation of $D_{\varepsilon}^{k-i-1}$ we may assume that these are fixed standard framings at each point in $\theta\left(S^{i}\right)=S^{i} \times$ $0 \subset K$.

(j) By condition (2) the framing $\eta$ of $T D_{\varepsilon}^{n} \times D^{i+1} \times 0$ corresponds to the last $n$ vectors of the framing of $\gamma\left(D^{i+1}\right)=0 \times D^{i+1} \times 0$. Since $D_{\varepsilon}^{n}$ was rotated in (f) and (g) we cannot assume that $\eta$ is the standard framing of $T D_{\varepsilon}^{n}$, but this is irrelevant.

We are now ready to construct the realization of the two-stage surgery $(\theta, c, \eta, \zeta, \gamma)$. First note that the projection $\pi: A_{1}^{q}(f) \rightarrow K$ is an immersion and thus an embedding in a neighborhood of $\theta\left(S^{i}\right)$. By making $\varepsilon$ smaller we can assume that $\pi$ : $A_{1}^{q}(f) \rightarrow K$ is an embedding. Since the disk $D^{i+1}$ is tangent to $A_{1}^{q}(f)$ along $S^{i}$ we can assume by a small deformation of $f$ that $A_{1}^{q}(f)$ lies in $0 \times \bar{D}^{i+1} \times 0$ near $\theta\left(S^{i}\right)=0 \times S^{i} \times 0$. By (i) we may assume that $\Sigma^{q}(h)=\pi^{-1}\left(0 \times S^{i} \times D_{\varepsilon}^{k-q-i}\right)$ and that

$$
\xi\left(\pi^{-1}\left(0,\left(1-x_{q}\right) y,\left(0, x_{1}, \ldots, x_{q-1}\right)\right)\right)=\left(x_{1}, \ldots, x_{q}\right)
$$

for all $y \in S^{i}$ and $\left(x_{1}, \ldots, x_{q}\right)$ in a neighborhood of 0 in $\mathbf{R}^{q}$.

The first step in the deformation of $h$ is to produce two $S^{i} \times S^{k-i-1}$ components of $A_{2}^{q-1}$ points inside the sphere $\theta\left(S^{i}\right)$ as indicated in Figure L. This is accomplished by "rotating" the framed deformation indicated in Figures $\mathrm{E}$ and $\mathrm{F}$ of $\S 4$. For each $y \in S^{i}$ we perform the indicated framed deformation of the $(k-i)$ - 
parameter family of functions $f_{t}$, where $t=\left(\left(1-x_{q}\right) y, \quad\left(\left(X_{q+1}, \ldots, x_{k-i}\right)\right.\right.$, $\left.\left.\left(x_{1}, \ldots, x_{q-1}\right)\right)\right)$. Since this family is essentially independent of $y$ we can perform the same deformation at each $y$.

Since $i+1<n=\operatorname{codim}\left(\Sigma(f), D_{\varepsilon}^{n} \times K\right)$ we can assume that the outer $i$-sphere of $A_{2}^{q-1}$ points bounds an $(i+1)$-disk in the complement of $\Sigma(f)$ so that this $(i+1)$-disk is $C^{1}$-close to $0 \times D^{i+1} \times 0$ as indicated by dotted lines in Figure $\mathrm{M}$. The $i$-sphere of $A_{2}^{q-1}$ points can be cancelled along this $(i+1)$-disk by the birth-death cancellation lemma (A2.2). The result is shown in Figure N. This deformation is almost identical to the deformation indicated in Figures $\mathrm{H}$ and I of $\S 4$.

The sphere $\theta\left(S^{i}\right)$ now bounds an $(i+1)$-disk in $A_{1}^{q}(f)$ which we may assume is the standard disk $0 \times D^{i+1} \times 0$. By construction the vector $\xi$ is given by $\xi(x,(0, z))$ $=(z, *)$ for all $(x, z) \in D^{i+1} \times D_{\varepsilon}^{q-1}$ where $* \geqslant 0$ and $*=0$ only on $S^{i} \times D_{\varepsilon}^{q-1}$. By deforming the last vector $*$ of $\xi$ we can alter $\Sigma^{q}(h)$ by the desired surgery.
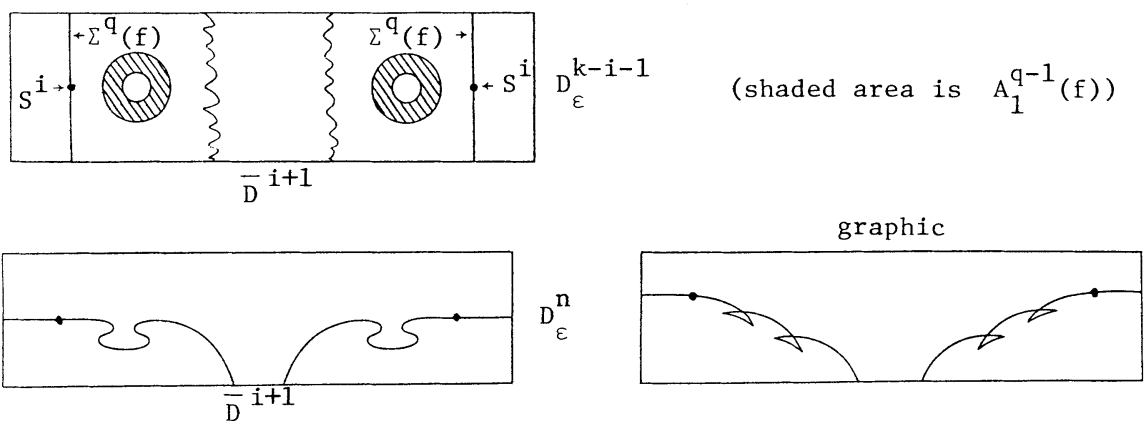

FIGURE L (create an $S^{i}$ of $A_{2}^{q-1}$ points)

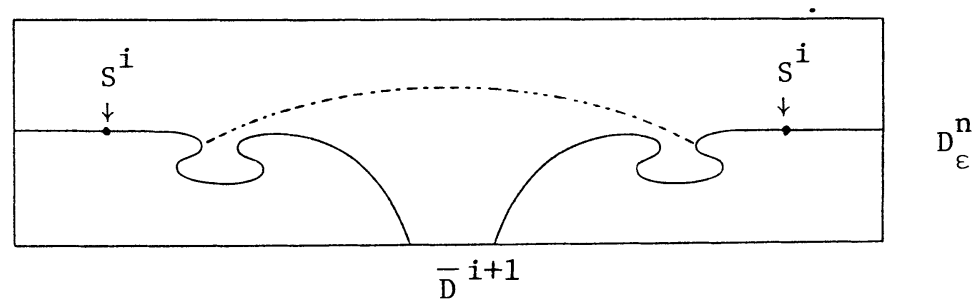

FIGURE M (cancellation path for the $S^{i}$ of $A_{2}^{q-1}$ points)
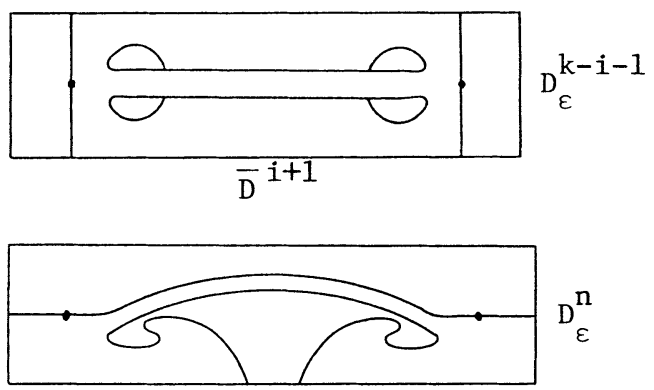

graphic

FIGURE N (after cancellation) 
It remains to show that $\gamma$ can be moved into standard form. More precisely

LEMMA 6.3. Under the conditions of Theorem 6.1 there exists a deformation $h^{u}$ of $h$ with support in the interior of $N \times \Delta^{k}$ so that

(a) $\Sigma^{q}\left(h^{u}\right)$ is an isotopy of $\Sigma^{q}(h)$,

(b) $\Sigma^{q}\left(h^{u}\right) \subset A_{1}^{q}\left(f^{u}\right)$, and

(c) there is a corresponding isotopy $\left(\theta^{u}, c^{u}, \eta^{u}, \zeta^{u}, \gamma^{u}\right)$ of the two-stage surgery so that $\gamma^{1}$ is in standard form.

Proof. Since $i \leqslant k-q \leqslant k-2$ we have that $\pi_{i+1}(O(n+k) / O(k))=0$. This means that $\gamma$ is framed isotopic rel $S^{i}$ to a framed embedding $\gamma^{\prime}: D^{i+1} \rightarrow N \times \Delta^{k}$ so that the composition $D^{i+1} \stackrel{\gamma^{\prime}}{\rightarrow} N \times \Delta^{k} \stackrel{\pi}{\rightarrow} \Delta^{k}$ is an immersion and the last $n$ vectors of the normal framing of $\gamma^{\prime}\left(D^{i+1}\right)$ are parallel to $N$ and coincide with the framing $\zeta$ of

$$
c^{*} \nu\left(A_{1}^{q}(N) \times \Delta^{k}, H(N) \times \Delta^{k}\right) \cong T N \times \Delta^{k} \mid \gamma^{\prime}\left(D^{i+1}\right) .
$$

On the other hand any framed immersion isotopy of $\pi \theta\left(S^{i}\right)$ in $\Delta^{k}$ can be realized by a deformation of $h$ which satisfies conditions (a) and (b) above. To show this it suffices to realize any "push" of $\pi \theta\left(S^{i}\right)$ as illustrated in Figure O. Any desired "push" can be realized by first creating a $(k-1)$-sphere of $A_{2}^{q-1}$ points in front of $\pi \theta\left(S^{i}\right)$ as shown in Figure $\mathrm{P}$ and then because $i+1<n=\operatorname{codim}\left(\Sigma(f), N \times \Delta^{k}\right)$ we can push the $A_{2}^{q-1}$ points forward as far as we want as indicated by the dotted arrows in Figure Q. Since every immersion isotopy of $\pi \theta\left(S^{i}\right)$ in $\Delta^{k}$ is equivalent to a finite sequence of pushes we are done.

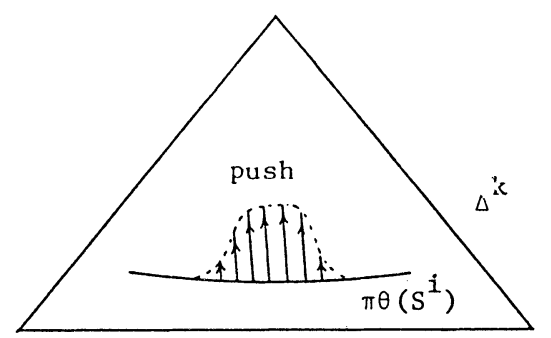

FIGURE O (pushing the obstruction set)

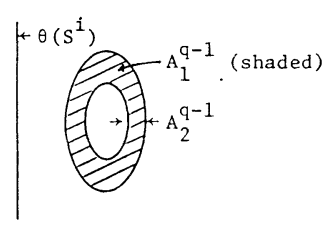

cross section:

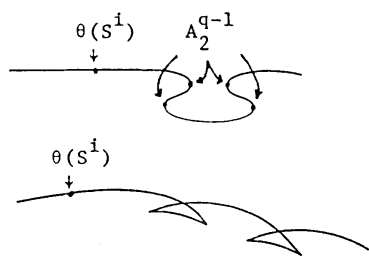

Figure P (preparing to push) 

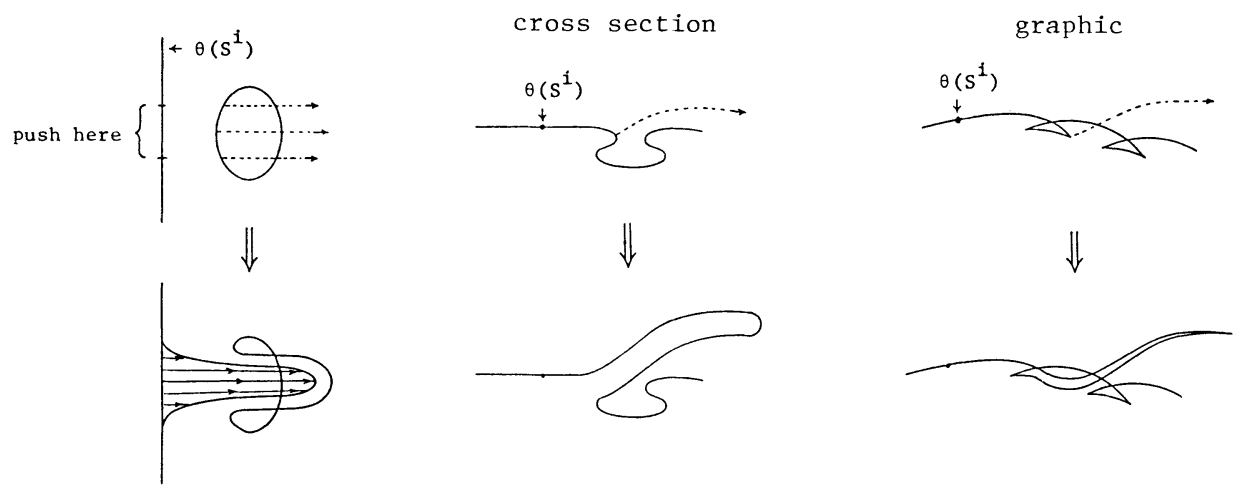

FIGURE Q (pushing $\left.\theta\left(S^{i}\right)\right)$

7. Proof of the theorem on semiframed functions. We are now ready to prove Theorem 3.8. The proof is analogous to the argument of [I2, §9].

We consider $\Delta^{k}$ to be the last $k$-face of $\Delta^{k+1}$ and we let $K^{k}$ denote the union of the other $k$-faces of $\Delta^{k+1}$. Then we have the following diagram of inclusion maps:

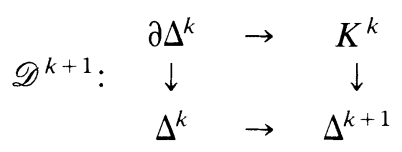

Let $(\tau, h): \mathscr{D}^{k+1} \rightarrow \mathscr{S}_{q}$ be a simplicial map of squares, where $\mathscr{S}_{q}$ is as given in Theorem 3.8. This means that $h$ is a simplicial map $h:\left(\Delta^{k}, \partial \Delta^{k}\right) \rightarrow$ $\left(\mathscr{L}_{q}^{\Delta}(N), \mathscr{L}_{q-1}^{\Delta}(N)\right)$ given by $h(t)=\left(f_{t}, \mathfrak{m}_{t}, \xi_{t}\right)$ and $\tau:\left(\Delta^{k+1}, K^{k}\right) \rightarrow\left(\Gamma_{q}^{\Delta}, \Gamma_{q-1}^{\Delta}\right)$ is a simplicial map with $\tau(t)=\left(\sigma_{t}, \mathfrak{m}_{t}^{\prime}, \xi_{t}^{\prime}\right)$, where $\sigma_{t}=\tilde{j}^{3} f_{t}, \mathfrak{m}_{t}^{\prime}=\mathfrak{m}_{t}$, and $\xi_{t}^{\prime}=\xi_{t}$ when $t \in \Delta^{k}$.

As before we have $\Sigma(f), W_{q}(f), \rho: W_{q}(f) \rightarrow B_{q}(N) \times \Delta^{k}, \Sigma^{q}(h), \Sigma(\sigma), W_{q}(\sigma)$, $\rho^{\prime}: W_{q}(\sigma) \rightarrow B_{q}(N) \times \Delta^{k+1}$, and $\Sigma^{q}(\tau)$. We may assume that $\tau$ and $h$ satisfy the transversality condition of Proposition 3.6 which makes the obstruction set $\Sigma^{q}(\tau)$ into a compact smooth $(k-q+1)$-manifold with boundary $\Sigma^{q}(h)$.

In order to find a null homotopy of the map $(\tau, h): \mathscr{D}^{k+1} \rightarrow \mathscr{S}_{q}$ it suffices to find a transverse smooth deformation of $(\tau, h)$ which is fixed on $K$ so that $\sum^{q}(\tau)$ dissappears. Such a deformation can be triangulated to give a simplicial null deformation of $(\tau, h)$. To prove Theorem 3.8 it suffices to construct such a null homotopy when $q \geqslant 2$ and $k \leqslant n$. By Proposition 3.7 we may assume that $k+1 \geqslant q$. In other words $n \geqslant k \geqslant q-1 \geqslant 2$.

These conditions imply $k \leqslant n+q-2$. Therefore we may use Theorem 4.3 to deform $\Sigma^{q}(h)$ into $A_{1}^{q}(f)$ by an isotopy. This extends easily to a deformation of the pair $(\tau, h)$ which is fixed over $K$. Thus we may assume that $\Sigma^{q}(h) \subset A_{1}^{q}(f)$. By Lemma 5.3 we may also assume that $\Sigma^{q}(\tau) \subset A_{1}^{q}(\sigma)$.

Let $g: \Sigma^{q}(\tau) \rightarrow I$ be a Morse function with $g^{-1}(0)=\partial \Sigma^{q}(\tau)=\Sigma^{q}(h)$. If $g$ has no critical points, then $\Sigma^{q}(\tau)$ is empty and we are done. Therefore by induction it suffices to deform $(\tau, h)$ and $g$ so that the number of critical points of $g$ decreases by 1 . 


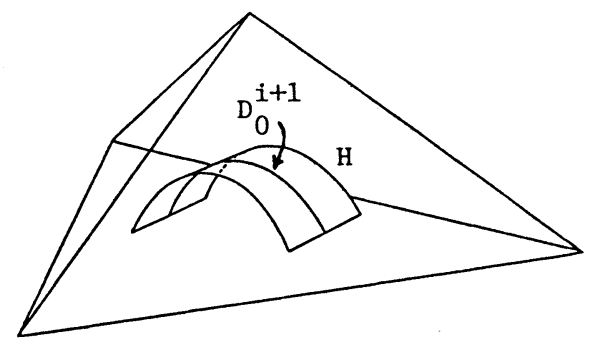

FIGURE R (an $(i+1)$-handle in the obstruction set)

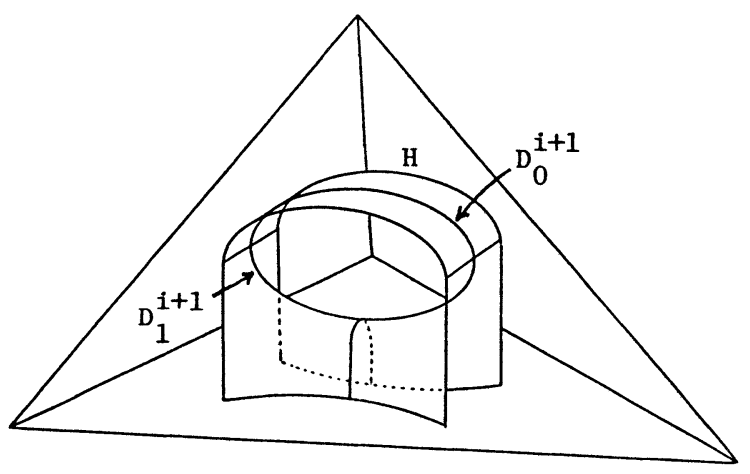

FIGURE S (the sphere $D_{0} \cup D_{1}$ )

Take a critical point of $g$ with minimal critical value. This corresponds to a handle $H$ with core $D_{0}^{i+1}$, where $i+1$ is the index of the critical point (see Figure R). Note that $i+1 \leqslant \operatorname{dim} \Sigma^{q}(\tau)=k-q+1 \leqslant k-1 \leqslant n-1$ and so $2(i+1) \leqslant$ $n+k-2$. Consequently $D_{0}^{i+1}$ is isotopic rel $\partial D_{0}^{i+1}$ to an embedding $\gamma: D^{i+1} \rightarrow$ $N^{n} \times \Delta^{k-1}$. Choosing arbitrary framings of the appropriate bundles over $D^{i+1}$ we get a two-stage admissible surgery $\left(\partial D_{0}^{i+1}, c, \eta_{1}, \eta_{2}, \gamma\right)$. By Theorem 6.1 this admissible surgery can be realized up to isotopy by a deformation of $h$. This deformation extends to a deformation of $(\tau, h)$ which alters the obstruction set to give Figure $\mathrm{S}$. The surgery on $S^{i}$ produces a new disk $D_{1}^{i+1}$ which is framed isotopic to $D_{0}^{i+1}$ by construction. This means that there is a two-stage admissible surgery on the new $\Sigma^{q}(\tau)$ with $\theta=D_{0}^{i+1} \cup D_{1}^{i+1}$. By Theorem 5.5 this surgery can be realized by a deformation of $(\tau, h)$ which is fixed over $\partial \Delta^{k+1}$. The end result is that the handle $H$ has been removed and a new Morse function $g^{\prime}$ on $\Sigma\left(\tau^{\prime}\right)$ exists with one fewer critical point than $g$. This proves Theorem 3.8.

8. Proof of the main theorem. In this section we prove the following.

THEOREM 8.1. The inclusion map $\mathscr{L}^{\Delta}(N) \rightarrow \mathscr{L}_{1}^{\Delta}(N)$ induces a monomorphism on $\pi_{k-1}$ for $k \leqslant n$. 
We first show that this implies the main theorem (1.6). For this we consider the following diagram:

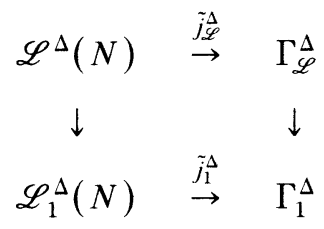

Since $\tilde{j}_{1}^{\Delta}$ is $n$-connected by Theorem 3.8 and $\Gamma_{\mathscr{L}}^{\Delta} \simeq *$ by Theorem 2.4 , the map $\mathscr{L}^{\Delta}(N) \rightarrow \mathscr{L}_{1}^{\Delta}(N)$ is trivial on $\pi_{k-1}$ for $k \leqslant n$. This together with Theorem 8.1 imply that $\mathscr{L}^{\Delta}(N)$ must be $(n-1)$-connected.

Proof of 8.1. By the long exact homotopy sequence this theorem is equivalent to the statement that the boundary map $\pi_{k}\left(\mathscr{L}_{1}^{\Delta}(N), \mathscr{L}^{\Delta}(N)\right) \rightarrow \pi_{k-1} \mathscr{L}^{\Delta}(N)$ is trivial for $k \leqslant n$. To prove this we let $h(t)=\left(f_{t}, \mathfrak{m}_{t}, \xi_{t}\right), t \in \Delta^{k}$, be a smooth family of 1 -semiframed functions with $h(t)$ framed for $t \in \partial \Delta^{k}$. We will then modify $f_{t}$ for $t \in$ int $\Delta^{k}$ by a deformation $f_{t}^{u}$ involving dovetails and butterflies so that the resulting function $f_{t}^{1}$ admits a framed structure extending the framed structure already given for $t \in \partial \Delta^{k}$.

As before the first step is to move the obstruction set $\Sigma^{1}(h)$ into the single index $A_{1}^{1}(f)$. This follows from Theorem 4.3 if $k<n$. For the case $k=n$ we have the following special argument. We proceed as in the proof of Theorem 4.3. Suppose by induction the $\Sigma^{1}(h) \subset W_{1}^{i}$ where $2 \leqslant i \leqslant n .\left(W_{1}^{i}=A_{2}^{0}(f) \cup \cdots \cup A_{1}^{i}(f)\right.$.) Then we must show that $\Sigma^{1}(h)$ can be moved into $W_{1}^{i-1}$. Let $V=A_{1}^{i}(f) \cup A_{2}^{i-1}(f)$ and $J=\Sigma^{1}(h) \cap V$. Take a cell decomposition of $(J, \partial J)$ so that each cell maps homeomorphically to a closed disk in $\Delta^{k}$. Then as in the proof of Theorem 4.3 we may remove any $p$-cell of $(J, \partial J)$ if $p \leqslant n-2$. The problem comes when $k=n$ and $p=k-1$. In this case Lemma 4.2 fails. We shall examine this case in detail. We may assume that all cells of dimension $\leqslant n-2$ have been removed so $J$ will be a disjoint union of $(k-1)$-disks whose projections to $\Delta^{k}$ are topological embeddings. Also $k=n \geqslant 2$.

We now proceed as in the proof of Lemma 4.2 (and see where it fails.) We are looking at an embedding $e:\left(D^{k-1}, S^{k-2}\right) \rightarrow(V, \partial V)$ which is parallel to a disk in $J$. We can perform the generalized radiation construction to arrive at Figure $\mathrm{H}$ and we can cancel the $(k-2)$-sphere of $A_{2}^{i-2}$ points to arrive at Figure I (since $k-2 \leqslant n$ - 2). We cannot however apply the lens replacement lemma (A4.5) to arrive at Figure J.
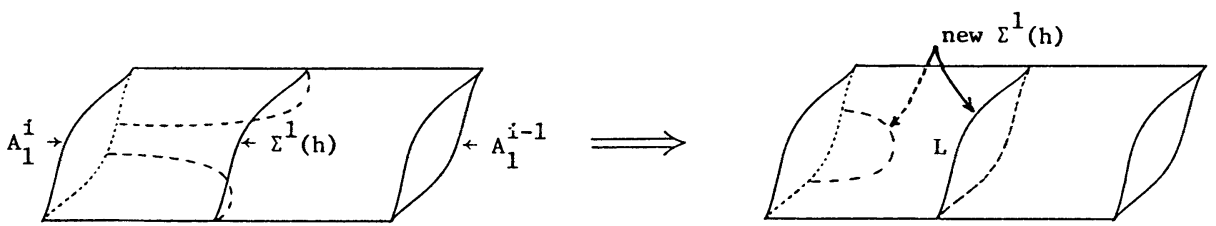

Figure T (deformation of $\left.\Sigma^{1}(h)\right)$ 

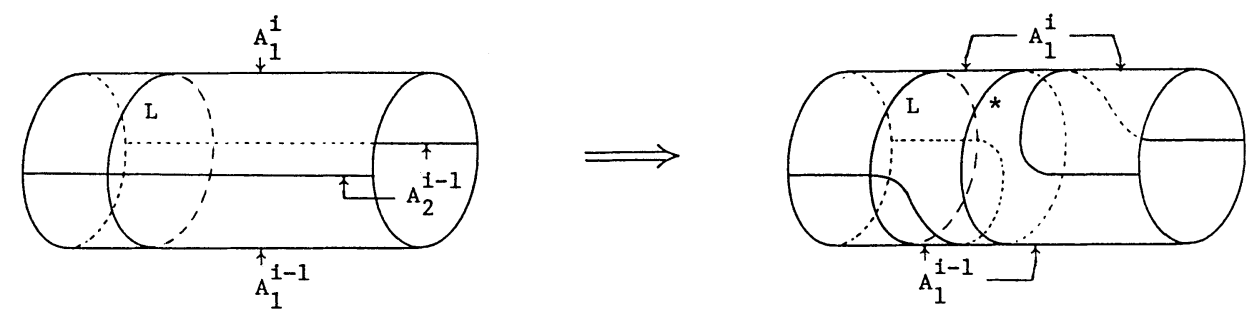

Figure U (imitate proof of lens replacement)

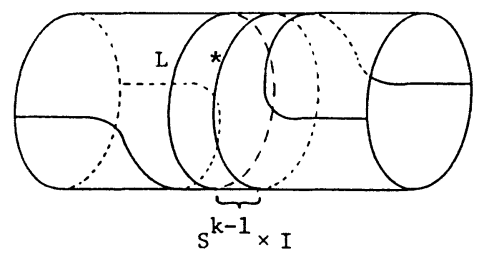

Figure V (eliminate $L$ )

We now take the $(k-1)$-lens which we cannot eliminate and perform the concordance of $\Sigma^{1}(h)$ shown in Figure T (graphics are drawn). The new $\Sigma^{1}(h)$ will have the same $J$ (intersection with $A_{1}^{i}(f)$ ) as before but one component $L$ of $\Sigma^{1}(h)$ will be a null homotopic $(k-1)$-lens. The concordance will be performed by a deformation of the vector $\xi_{1}$. As in $\S 3$ and the proof of Lemma 4.1 we are looking at $\Sigma^{1}(h)=s^{-1}$ (zero section), where $s$ is a variable section of some line bundle over $W_{1}(f)$. It is easy to change $s^{-1}$ (zero section) as desired since this line bundle is trivial over the $(k-1)$-lens by construction.

As in the proof of the framed lens lemma (A4.6) we may pass through a $(k-2)$-sphere of $A_{4}$ points and two $(k-1)$-disks of $A_{3}$ points to arrive at the family of functions shown in Figure U. Away from $L$ we have a "framing up to sign" where the $(k-1)$-sphere of $A_{2}^{i-1}$ points marked $*$ in Figure $\mathrm{U}$ is "negatively framed" in the sense that the vector $\xi^{i}$ points in the negative cubic direction of $D^{2} f$ along this set. By the isotopy lemma (4.1) we may move the component $L$ of $\Sigma^{1}(h)$ to a sphere parallel to this negatively framed $A_{2}^{i-1}$ sphere and disjoint from $A_{2}^{i-1}(f)$.

In the region $S^{k-1} \times I$ between these two spheres where $\xi$ is not framed the signs of $\xi_{i}$ and $\xi_{1}$ are both reversed as we move in the $I$-direction. Thus we may change the two vectors in this region so that they rotate instead of changing sign. $(-I$ is homotopic to $+I$ in $O(2)$.) Then the sphere $L$ disappears and the negatively framed sphere of $A_{2}^{i-1}$ points becomes (positively) framed. The end result is that one component of $J$ is gone. Thus we may eliminate $J$ and by induction we can move $\Sigma^{1}(h)$ into $A_{1}^{1}(f)$.

Let $S=\Sigma^{1}(h) \subset A_{1}^{1}(f)$. The negative eigenspace of $D^{2} f$ is one dimensional along $S$. This gives a line bundle $\eta$ over $S$ which is isomorphic to the normal bundle of $S$ in $\Delta^{k}$ by Proposition 3.6(b). Thus we may apply the dovetail lemma (A3.4) and we 
get a deformation of $f$ with support in an arbitrarily small neighborhood of $S$ which passes through a $k-1$ parameter family of negative dovetails along $S=\Sigma^{1}(h)$ and which makes $f$ framed.

\section{APPENDIX}

This appendix explains some elementary facts about $A_{1}, A_{2}, A_{3}$, and $A_{4}$ singularities used in the main part of this paper.

A0. Preliminary definitions. If $n, s$ are nonnegative integers, then let $J^{s}(n)$ denote the truncated polynomial algebra $J^{s}(n)=\mathbf{R}\left[x_{1}, \ldots, x_{n}\right] /\left(x_{1}, \ldots, x_{n}\right)^{s+1}$. Let $\tilde{J}^{s}(n)$ denote the unique maximal ideal in $J^{s}(n)$. Then $\tilde{J}^{s}(n)=\left\{f \in J^{s}(n) \mid f(0)=0\right\}$. If $N$ is a smooth $\left(C^{\infty}\right) n$-manifold, then let $J^{s}(N)$ denote the bundle of $s$-jets of maps $N \rightarrow \mathbf{R}$. This is a bundle over $N$ with fiber $J^{s}(n)$ and structure group $G^{s}(n)=$ Aut $_{\mathbf{R}} J^{s}(n)$. Let $\tilde{J}^{s}(N)$ denote the subbundle of $J^{s}(N)$ corresponding to $\tilde{J}^{s}(n)$. Note that any $G^{s}(n)$-invariant subspace of $J^{s}(n)$ determines a subbundle of $J^{s}(N)$. (Invariance under $G^{s}(n)$ is equivalent to invariance under change of coordinates.)

Suppose that $N$ has a Riemannian metric. Then at each $x \in N$ we have the exponential map $\exp _{x}: T_{x} N, 0 \rightarrow N, x$ which is defined in a neighborhood of 0 if $x \in$ int $N$. Given any orthonormal tangent $n$-frame at $x$ we get a linear isomorphism $\mathbf{R}^{n} \cong T_{x} N$. The composition $\mathbf{R}^{n}, 0 \rightarrow T_{x} N, 0 \rightarrow N, x$ induces an isomorphism between $J^{s}(n)$ and the fiber of $J^{s}(N)$ over $x$. Thus the structure group of $J^{s}(N) \mid$ int $N$ is reduced to $O(n) \subset G^{s}(n)$. This reduction of the structure group can be extended to $\partial N$ by attaching an exterior collar to $N$. Since $N$ is the closure of int $N$ this extension is unique. Given a metric on $N$ any $O(n)$-invariant subspace of $J^{s}(n)$ determines a subbundle of $J^{s}(N)$.

If $f: N \rightarrow \mathbf{R}$ is a smooth map then we get a smooth section $j^{s} f$ of $J^{s}(N)$ over $N$ called the s-jet of $f$. When $N^{n} \subset \mathbf{R}^{n}, J^{s}(N)=N \times J^{s}(n)$ and $j^{s} f(x)=(x$, the $s$-order Taylor polynomial of $f$ at $x)$. The normalized s-jet of $f$ is defined to be the section $\tilde{j}^{s} f$ of $\tilde{J}^{s}(N)$ given by $\tilde{j}^{s} f(x)=j^{s} f(x)-f(x)$.

The singular set of $f$ is the inverse image under $\tilde{j}^{s} f$ of a subbundle of $\tilde{J}^{s}(N)$. We use the following notation:

$\Sigma(f)=$ singular set of $f$,

$\Sigma(n)=\tilde{J}^{s}(n)^{2} \subset \tilde{J}^{s}(n) \subset J^{s}(n)$,

$\Sigma(N)$ is the subbundle of $\tilde{J}^{s}(N)$ with fiber $\Sigma(n)$.

This paper considers only four types of singularities. These are the Morse, birth-death, dovetail, and butterfly singularities which are $A_{1}, A_{2}, A_{3}, A_{4}$ respectively in the following definition.

Definition 0.1. $x_{0} \in$ int $N$ is an $A_{k}$-singularity of $f$ if there exists a system of local coordinates $x_{1}, \ldots, x_{n}$ for $N$ centered at $x_{0}$ so that

$$
f= \pm x_{1}^{k+1}+\sum_{j=2}^{n} \pm x_{j}^{2}+f\left(x_{0}\right)
$$

in a neighborhood of $x_{0}$.

If $k$ is odd and $k \geqslant 3$, then the sign in front of $x_{1}^{k+1}$ is intrinsic (invariant under change of coordinates) and is called the sign of $x_{0}$. If $k$ is even we take the sign in front of $x_{1}^{k+1}$ to be positive. In this case the orientation of the $x_{1}$-axis is intrinsic. 
The index of $D^{2} f\left(x_{0}\right)$ is called the index of $x_{0}$. If $k \geqslant 2$ the index is the same as the number of negative signs in the summation $\sum \pm x_{j}^{2}$. We sometimes write ${ }^{\varepsilon} A_{k}^{i}$ to denote an $A_{k}$ singularity with sign $\varepsilon$ and index $i$. We also use the following notation:

$A_{k}(n)=\left\{f \in \Sigma(n) \mid 0\right.$ is an $A_{k}$-singularity of $\left.f\right\}$,

$A_{k}(N)=$ the subbundle of $\tilde{J}^{s}(N)$ with fiber $A_{k}(n)$,

$A_{k}(f)=\tilde{j}^{s} f^{-1} A_{k}(N)=$ the set of all $A_{k}$-singularities of $f$.

We use the analogous notation for $A_{k}^{i},{ }^{\varepsilon} A_{k}^{i}$. We need $s \geqslant k+1$ in order for the polynomial in 0.1 to lie in $J^{s}(n)$.

DEFINITION 0.2. The standard unfolding of an $A_{k}$-singularity is given as $p_{t}$ : $\mathbf{R}^{n} \rightarrow \mathbf{R}, t \in \mathbf{R}^{k-1}$,

$$
p_{t}(x)=x_{1}^{k+1}+t_{1} x_{1}^{k-1}+t_{2} x_{1}^{k-2}+\cdots+t_{k-1} x_{1}+\sum_{j=2}^{n} \pm x_{j}^{2} .
$$

This family of functions has the property that its normalized $(k+1)$-jet $\tilde{j}^{k+1} p_{t}$ : $\mathbf{R}^{n} \times \mathbf{R}^{k-1} \rightarrow \tilde{J}^{k+1}(n)$ meets $A_{k}(n)$ transversely at the single point $(0,0) \in \mathbf{R}^{n} \times$ $\mathbf{R}^{k-1}$. Conversely any smooth family of maps $f_{t}: \mathbf{R}^{n} \rightarrow \mathbf{R}, t \in \mathbf{R}^{k-1}$, so that $\tilde{j}^{k+1} f_{t}$ meets $A_{k}(n)$ transversely at $(0,0)$ is equivalent to $p_{t}$ plus a function of $t$ in some neighborhood of $(0,0)$ after some change of coordinates $(\phi, \psi)$ making the following diagram commute:

$$
\begin{array}{ccc}
\mathbf{R}^{n} \times \mathbf{R}^{k-1},(0,0) & \stackrel{\phi}{\rightarrow} & \mathbf{R}^{n} \times \mathbf{R}^{k-1},(0,0) \\
\downarrow & & \downarrow \\
\mathbf{R}^{k-1}, 0 & \stackrel{\psi}{\rightarrow} & \mathbf{R}^{k-1}, 0
\end{array}
$$

$(f=p \phi+g$ near $(0,0)$.

The standard unfoldings of $A_{1}, A_{2}, A_{3}, A_{4}$ singularities have been thoroughly studied by catastrophe theorists (see for example [G]).

A1. Morse singularities. The very well-known Morse lemma says that $A_{1}$-singularities, as defined in 0.1 , are exactly those which are nondegenerate (i.e. $D^{2} f$ is nonsingular). This implies that $A_{1}$ points are always "transverse" in the sense that for any smooth $f: N \rightarrow \mathbf{R}, \tilde{j}^{s} f: N \rightarrow \tilde{J}^{s}(N)$ meets $A_{1}(N)$ transversely if $s \geqslant 2$. In fact we have

Proposition 1.1. Suppose that $f_{t}: N \rightarrow \mathbf{R}, t \in D^{k}$, is a smooth family of smooth functions which are nonsingular on $\partial N$ and $s \geqslant 2$. Then

(a) $\tilde{j}^{s} f: N \times D^{k} \rightarrow \tilde{J}^{s}(N)$ meets $A_{1}(N)$ transversely.

(b) $A_{1}(f)$ is a smooth $k$-submanifold of $N \times D^{k}$.

(c) The composition $A_{1}(f) \subset N \times D^{k} \rightarrow D^{k}$ is an embedding.

The parameterized version of the Morse lemma is "twisted" because of the fact that certain bundles may not be trivial.

Proposition 1.2 (PARAMETERIZEd MORSE LEMMA-TWISTED FORM). Let $f_{t}: N \rightarrow$ $\mathbf{R}, t \in D^{k}$, be as in 1.1. Let $K$ be a compact subset of $A_{1}^{i}(f)$. Then there exist two smooth vector bundles $E_{+}^{n-i}, E_{-}^{i}$ over $K$ (namely the $(+),(-)$ eigenspace bundles of 
$D^{2} f$ ) with structure groups $O(n-i), O(i)$, a neighborhood $U$ of the zero section of $K$ in $E_{+} \oplus E_{-}$, and a smooth embedding $\phi: U \rightarrow N \times D^{k}$ having the following properties.

(a) The following diagram commutes where $\psi$ is the restriction of $N \times D^{k} \rightarrow D^{k}$ to $K$.

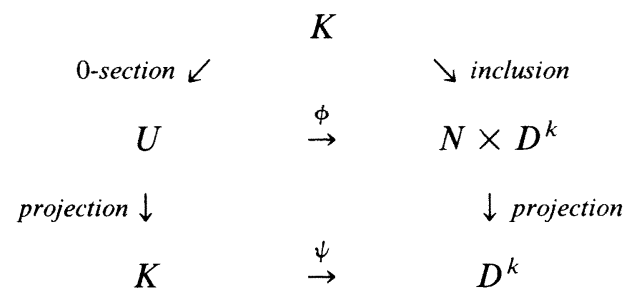

(b) If $(x, y) \in U \subset E_{+} \oplus E_{-}$lies over $z \in K$, then $f \phi(x, y)=f(z)+\|x\|^{2}-\|y\|^{2}$.

Proof. Generalize any proof of the standard Morse lemma.

COROllary 1.3 (PARAMETERIZED MORSE LEMMA-UNTWISTED VERSION). If the $n-i$ and $i$-plane bundles over $K$ given by the $(+)$ and $(-)$ eigenspaces of $D^{2} f$ are trivial, then there exists an open neighborhood $V$ of 0 in $\mathbf{R}^{n}$ and a smooth family of embeddings $\phi_{z}: V \rightarrow N, z \in K$, so that

(1) $\phi_{z}(0,0)=x$ if $z=(x, t) \in K \subset N \times D^{k}$.

(2) $f_{t} \phi_{(x, t)}(u, v)=f_{t}(x)+\|u\|^{2}-\|v\|^{2}$ for all $((x, t),(u, v)) \in K \times V$.

A2. Birth-death singularities. The standard unfolding of an $A_{2}^{i}$-singularity (birthdeath point) is $p_{t}=x_{1}^{3}+t x_{1}+\sum_{j=2}^{n} \pm x_{j}^{2}$. The singular set,

$$
\Sigma(p)=\left\{(x, t) \in \mathbf{R}^{n} \times \mathbf{R} \mid t=-3 x_{1}^{2}, x_{j}=0 \text { for } j \geqslant 2\right\}
$$

and the graphic are indicated in Figure A. (The graphic of a family of functions $f_{t}$ is defined to be the set of all pairs $(c, t)$ so that $c$ is a critical value of $f_{t}$.)

The $+t$ direction is called the cancellation direction because two Morse $\left(A_{1}\right)$ critical points are being cancelled as $t$ goes from $<0$ to $>0$. Under very special conditions we can use birth-death points to cancel two Morse points. Under very general conditions an $A_{2}$ point can be used to create pairs of $A_{1}$ points. In fact given any smooth $f: N \rightarrow \mathbf{R}$ and any $x_{0} \in$ int $N-\Sigma(f)$ there is a deformation of $f$ with support in an arbitrarily small neighborhood of $x_{0}$ which creates two $A_{1}$ critical

singular set:

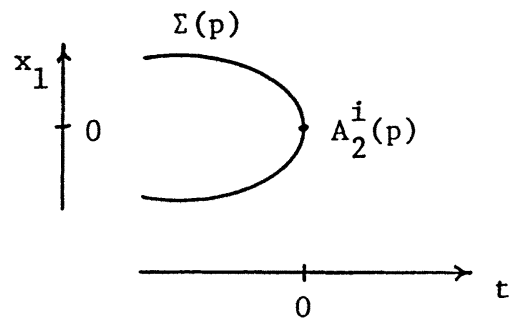

graphic:

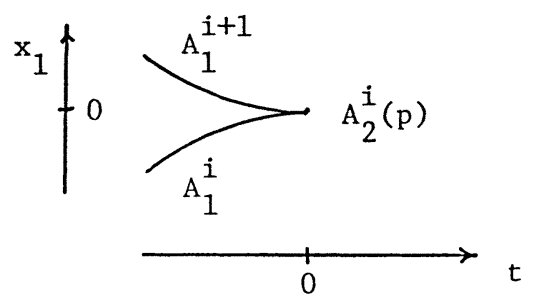

Figure A (standard unfolding of $A_{2}^{i}$ ) 
points of index $i$ and $i+1$ by passing through one $A_{2}^{i}$-singularity (assuming $0 \leqslant i<n=\operatorname{dim} N)$. This deformation is constructed by "pasting in" the deformation $p_{t}$ using the following lemma. (Take $B$ sufficiently small in Lemma 2.1 so that there is an embedding $\psi:(B, 0) \rightarrow\left(N, x_{0}\right)$ with $f \psi(x)=f\left(x_{0}\right)+x_{1}$ for all $x \in B$. Then $f^{u}$ can be given by $f^{u}(y)=f(y)$ if $y \notin \psi(B)$ and $f^{u} \psi(x)=f\left(x_{0}\right)+p_{t} \phi_{t}(x)$, where $t=\delta(1-2 u)$.)

Lemma 2.1 (PASTING LemMa FOR $A_{2}$ POINTS). Let $p_{t}: \mathbf{R}^{n} \rightarrow \mathbf{R}, t \in \mathbf{R}$, be as above, let $U$ be a neighborhood of 0 in $\mathbf{R}^{n}$, and let $\varepsilon>0$. Then there exists a compact neighborhood $B$ of 0 in $U$ and a smooth family of embeddings $\phi_{t}: B \rightarrow U, t \in[-\delta, \delta]$, where $0<\delta<\varepsilon$ so that the following conditions are satisfied.

(1) $\Sigma\left(p_{t}\right) \subset$ int $\phi_{t}(B)$ for all $t \in[-\delta, \delta]$.

(2) $p_{t} \phi_{t}(x)=x_{1}$ if either $x$ is close to $\partial B$ or $t$ is close to $\delta$.

Proof. This follows trivially from [I2, 4.5].

Suppose that $f_{t}: N \rightarrow \mathbf{R}, t \in D^{k}$, is a smooth family of smooth maps so that $f_{t}$ has no singularities on $\partial N$ and only $A_{1}$ and $A_{2}$ singularities in int $N$ for all $t$. Suppose also that $\tilde{j}^{3} f_{t}: N \times D^{k} \rightarrow \tilde{J}^{3}(N)$ and $\tilde{j}^{3} f_{t} \mid N \times S^{k-1}$ are transverse to $A_{2}(N)$. (This is not automatic as in the $A_{1}$ case.) Then $\Sigma(f)$ is a smooth $k$-submanifold of $N \times D^{k}$ and $A_{2}(f)$ is a smooth $(k-1)$-submanifold of $\Sigma(f)$ with $A_{2}(f)$ $\subset \Sigma(f) \subset N \times S^{k-1}$. The singular set $\Sigma(f)$ will look like (Figure A) $\times A_{2}(f)$ in a neighborhood of $A_{2}(f)$ and the composition $A_{2}(f) \subset N \times D^{k} \rightarrow D^{k}$ will be a codimension 1 immersion with a preferred orientation of its normal bundle given by the cancellation direction. A more precise description is as follows.

LEMMA 2.2 (NORMAL FORM LEMMA FOR $A_{2}$-SINGUlarities). Let $f_{t}: N \rightarrow \mathbf{R}$, $t \in D^{k}$, be as above. Let $K$ be a compact subset of $A_{2}^{i}(f)$. Then there exist

(1) smooth vector bundles $E_{+}^{i}, E_{-}^{n-i-1}$ over $K$ with orthogonal structure groups $O(i)$, $O(n-i-1)$ (i.e. the $(+),(-)$ eigenspace bundles of $D^{2} f$ with respect to some metric),

(2) a neighborhood $U$ of the zero section in $E_{+} \oplus E_{-}$,

(3) $\varepsilon, \delta>0$,

(4) a smooth immersion $\psi: K \times(-\delta, \delta) \rightarrow D^{k}$ (given by the cancellation direction of $\left.\nu\left(K, D^{k}\right)\right)$,

(5) a smooth embedding $\phi: U \times(-\varepsilon, \varepsilon) \times(-\delta, \delta) \rightarrow N \times D^{k}$ (parameterizing $a$ neighborhood of $K$ ), and

(6) a smooth function $g: K \times(-\delta, \delta) \rightarrow \mathbf{R}$ satisfying the following:

(a) The diagram

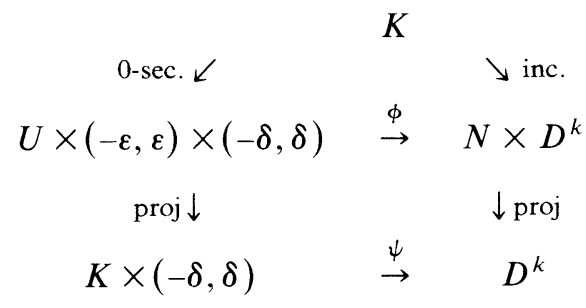

commutes. 
(b) If $x, y \in E_{+}, \quad E_{-}$lie over $z \in K$, and $u \in(-\varepsilon, \varepsilon), \quad v \in(-\delta, \delta)$ then $f \phi(x, y, u, v)=g(z, v)+u^{3}-u v+\|x\|^{2}-\|y\|^{2}$.

Remarks. (i) $E_{+} \oplus E_{-} \oplus(K \times \mathbf{R}=$ trivial bundle over $K)$ is isomorphic to the tangent bundle of $N$ over $K$.

(ii) If $E_{+}, E_{-}$are trivial bundles, then we get the obvious analogue of 1.3.

Proof. This follows easily from 1.2. See also [C and I4].

Let

$$
\beta: A_{2}^{i}(n) \rightarrow O(n) /(O(i) \times S O(1) \times O(n-i-1))
$$

be the map which sends the singularity $f \in A_{2}^{i}(n)$ to the $-, 0,+$ eigenspaces of $D^{2} f(0)$ together with the natural orientation of the 0 eigenspace of $D^{2} f(0)$. (Then $\beta$ is a homotopy equivalence.) Since $\beta$ is $O(n)$-equivariant we get a bundle map $\beta_{\mathfrak{m}}$ : $A_{2}^{i}(N) \rightarrow B_{i, 1}(N)$ if we choose a metric $\mathrm{m}$ on $N$. Here $B_{i, 1}(N)$ is the bundle over $N$ with fiber $O(n) /(O(i) \times S O(1) \times O(n-i-1))$ associated with the tangent bundle of $N$.

Proposition 2.3 (BirTh - DeATh CANCEllation lemma). Let $f_{t}: N \rightarrow \mathbf{R}, t \in D^{k}$, be a family of smooth functions with only $A_{1}$ and transverse $A_{2}$ singularities as discussed above. Let $\mathrm{m}_{t}$ be a family of metrics on $N$ and let $\theta: S^{j} \rightarrow A_{2}^{i}(f)$ be an embedding with image in int $A_{2}^{i}(f)$ satisfying the following.

(a) $j \leqslant n-2$.

(b) There is a map c: $D^{j+1} \rightarrow B_{i, 1}(N) \times D^{k}$ so that $c \mid S^{j}=\beta \theta$, where $\beta: N \times D^{k}$ $\rightarrow B_{i, 1}(N) \times D^{k}$ is given by $\beta(x, t)=\left(\beta_{\mathfrak{m}_{t}}(x), t\right)$.
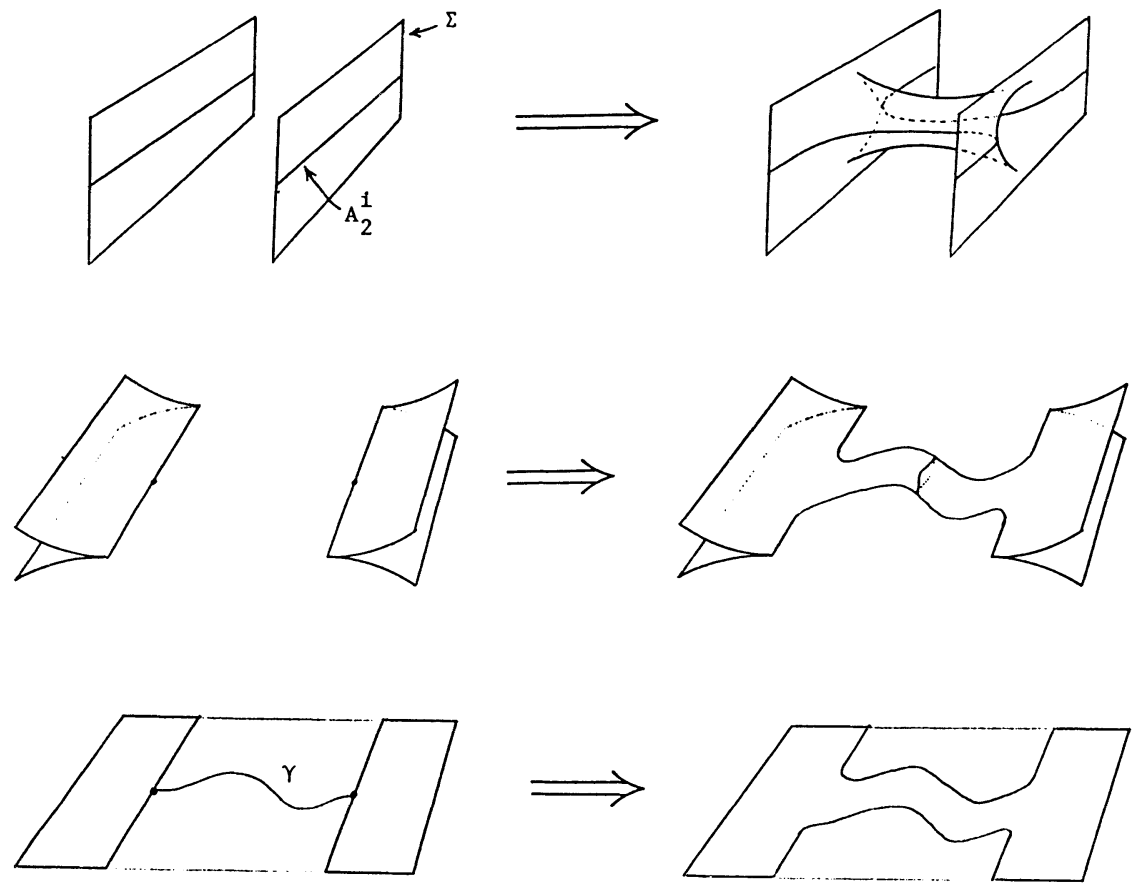

FiguRE B (surgery on a 0 -sphere in $A_{2}^{i}(f)$ ) 

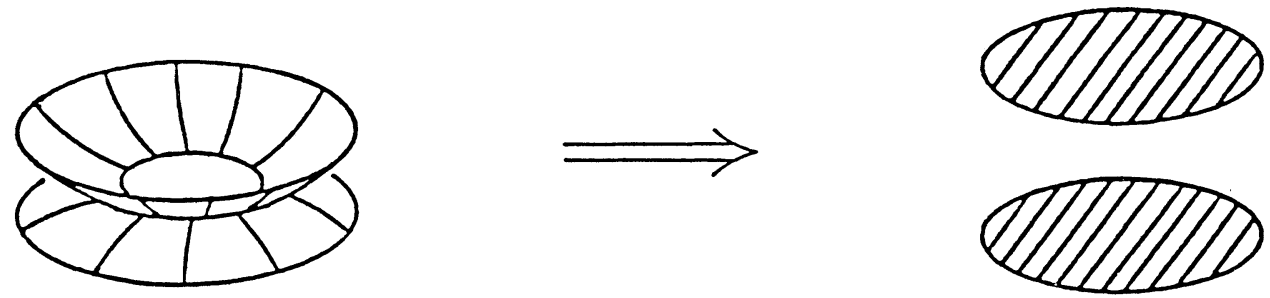

Figure C (surgery on a 1-sphere in $A_{2}^{i}(f)$-graphic only)

(c) The composition $D^{j+1} \stackrel{\subset}{\rightarrow} B_{i, 1}(N) \times D^{k} \stackrel{\mathrm{pr}}{\rightarrow} D^{k}$ is homotopic rel $S^{j}$ to an embedding $\gamma: D^{j+1} \rightarrow D^{k}$ so that the inward normal of $\gamma\left(S^{j}\right)$ in $\gamma\left(D^{j+1}\right)$ points in the cancellation direction.

Then $A_{2}^{i}(f)$ and $\Sigma(f)$ can be altered simultaneously by surgery on $\theta\left(S^{j}\right)$ along $(c, \gamma)$ as indicated in Figures B, C, D for the cases $j=0,1,-1$ respectively. (We assume that pr. $c=\gamma$.) In the special case when $i=-1$ this says that a " $k$-lens" (defined in 4.4 below) can be created at any nonsingular point assuming $n \geqslant 1$ (see Figure D).

ProOF. Let $\tilde{\gamma}$ be the composition $D^{j+1} \stackrel{\subset}{\rightarrow} B_{i, 1}(N) \times D^{k} \rightarrow N \times D^{k}$. Then we may assume that $\tilde{\gamma}$ is an embedding lying over $\gamma$ and by transversality (since $j+1<n$ ) we may assume that $\tilde{\gamma}\left(\right.$ int $\left.D^{j+1}\right)$ is disjoint from $\Sigma(f)$. Since $B_{i, 1}(N) \simeq$ $A_{2}^{i}(N)$, the map $c$ pulls back to give a null homotopy $\tilde{c}$ of $\tilde{j}^{3} f \theta: S^{j} \rightarrow A_{2}^{i}(N) \times D^{k}$ which covers $\tilde{\gamma}$. We are now in the situation of $[\mathbf{1 2}, \S 5]$. The sphere $\theta\left(S^{j}\right)$ can be cancelled by "inverse radiation." Since the neighborhood $U$ of Lemma 2.1 can be chosen arbitrarily small we do not have to create new singularities as in [12].

$\emptyset$

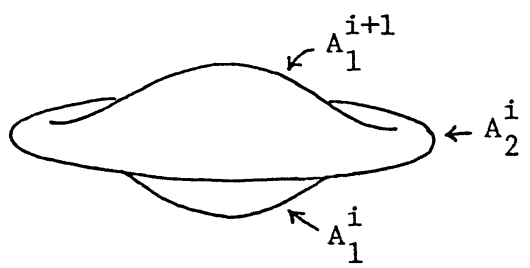

graphic
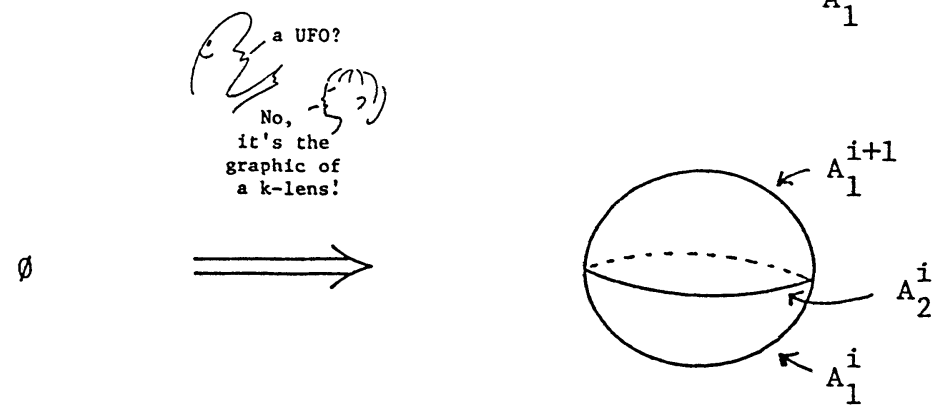

singular set

Figure D (surgery on a $(-1)$-sphere in $A_{2}^{i}(f)$ ) 
A3. Dovetails. The standard unfolding of an $A_{3}$-singularity (dovetail) is

$$
p_{t}= \pm x_{1}^{4}+t_{1} x_{1}^{2}+t_{2} x_{1}+\sum_{j=2}^{i+1} \pm x_{j}^{2}
$$

When the sign is positive this gives:

$$
\begin{gathered}
\Sigma(p)=\left\{(x, t) \in \mathbf{R}^{n} \times \mathbf{R}^{2} \mid t_{2}=-2 t_{1} x_{1}-4 x_{1}^{3}, x_{j}=0 \text { for } j \geqslant 2\right\}, \\
A_{2}(p)=\left\{(x, t) \in \Sigma(p) \mid t_{1}=-6 x_{1}^{2}\right\} .
\end{gathered}
$$

The singular set, graphic, and bifurcation diagrams are given in Figure E. The bifurcation diagram for $p$ is the set of all parameter values $t$ for which $p_{t}$ is not Morse. It can be shown that the bifurcation diagram of the standard unfolding of an $A_{k}$-singularity always looks like the graphic of the standard unfolding of an $A_{k-1}$-singularity. Figure $\mathrm{F}$ shows the graphic when the sign is negative.
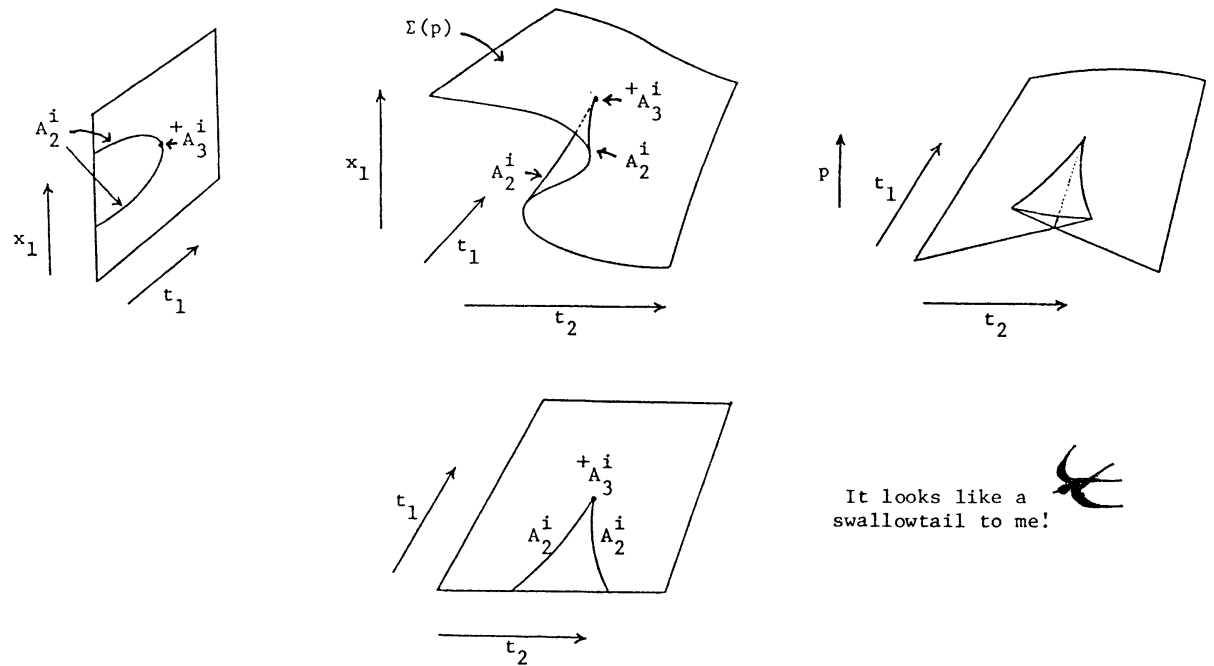

FIGURE E (standard unfolding of ${ }^{+} A_{3}^{i}$ )

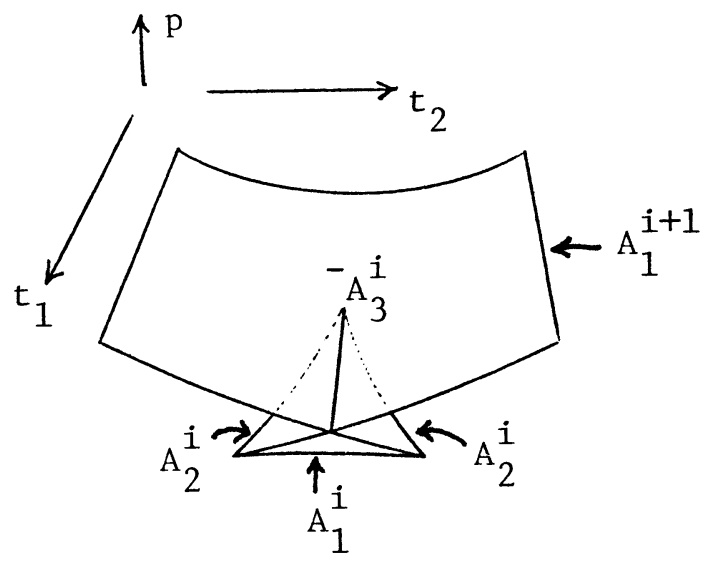

FIGURE F (standard unfolding of ${ }^{-} A_{3}^{i}$-graphic only) 

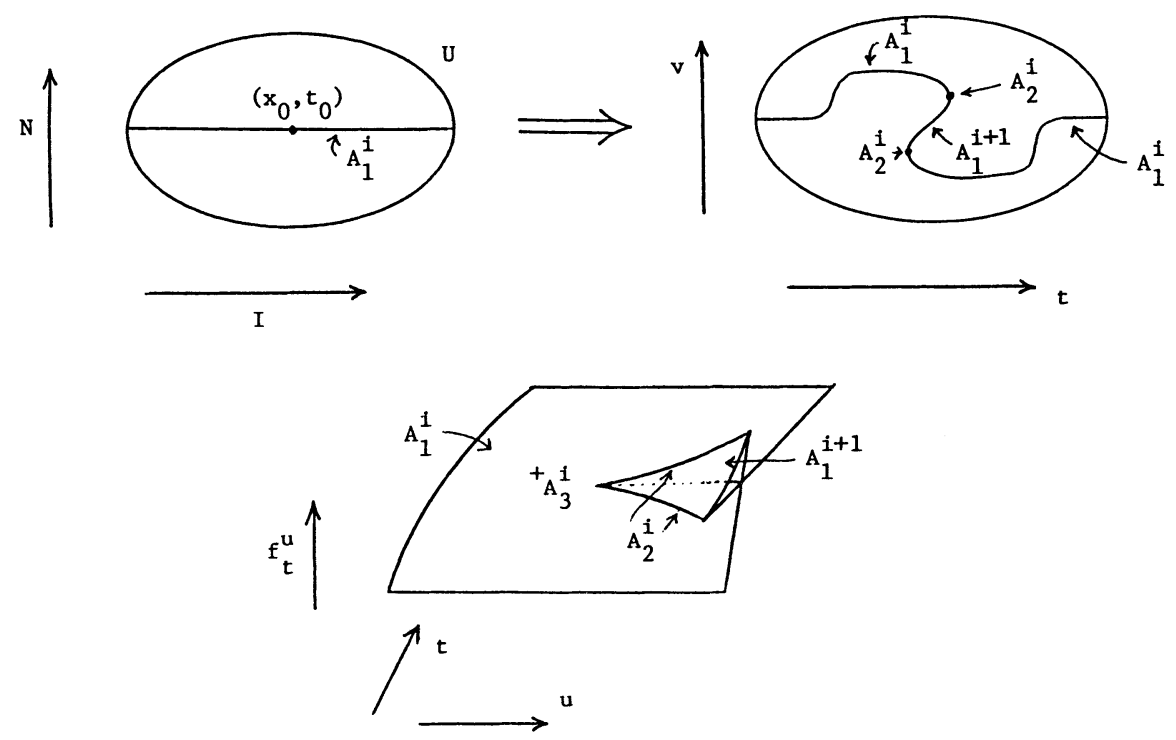

FIGURE G (creating two $A_{2}^{i}$ points)

As indicated in Figures $\mathrm{E}$ and $\mathrm{F}$ an $A_{3}^{i}$-singularity is a point at which two $A_{2}^{i}$-singularities are cancelled or created. In Figure $\mathrm{E}$ we call the positive $t_{1}$ direction the cancellation direction. In Figure $\mathrm{F}$ this is the negative $t_{1}$ direction. We will use $A_{3}$ points to create pairs of $A_{2}$ points and we will use families of $A_{3}$ points to create families of (pairs of) $A_{2}$ points. The simplest example of this is the following.

Lemma 3.1. Suppose that $f_{t}: N \rightarrow \mathbf{R}, t \in I$, is a one parameter family of smooth functions and $\mathfrak{m}_{t}, t \in I$, is a family of metrics on $N$. Let $\left(x_{0}, t_{0}\right) \in \operatorname{int}(N \times I)$ be an $A_{1}^{i}$-singularity of $f$ and suppose that $v$ is a nonzero tangent vector for $N$ at $x_{0}$ which lies in the $(+)$ eigenspace of $D^{2} f_{t_{0}}\left(x_{0}\right)$. (In particular $i<n=\operatorname{dim} N$.) Then there exists a deformation $f_{t}^{u}$ of $f_{t}$ with support in an arbitrarily small neighborhood $U$ of $\left(x_{0}, t_{0}\right)$ so that, inside $U, f_{t}^{u}$ looks just like the standard unfolding of an ${ }^{+} A_{3}^{i}$ singularity (see Figure $\mathrm{G}$ ), where $t=t_{2}, u=-t_{1}$, and $v$ gives the $x_{1}$-axis.

REMARK. If we assume that $i \geqslant 1$ and $v$ lies in the $(-)$ eigenspace of $D^{2} f_{t_{0}}\left(x_{0}\right)$ we get an analogous statement with the negative dovetail ${ }^{-} A_{3}^{i}$.

Proof. By the parameterized Morse lemma as given in 1.3 we can choose a system of local coordinates $x_{1}, \ldots, x_{n}$ for $N$ near $x_{0}$ which varies smoothly with $t$ near $t_{0}$ so that $f_{t}=g(t)+\sum_{j=1}^{n} \pm x_{j}^{2}$ near $\left(x_{0}, t_{0}\right)$. Furthermore we can choose these coordinates so that the vector $v$ points in the positive $x_{1}$ direction and the sign in front of $x_{1}^{2}$ is positive (the first condition implies the second). The standard deformation $p_{t}$ can now be "pasted in" using a partition of unity function and the following pasting lemma.

Lemma 3.2 (PASTING Lemma FOR $A_{3}$ POINTS). Let $p_{t}: \mathbf{R}^{n} \rightarrow \mathbf{R}, t \in \mathbf{R}^{2}$, be the standard unfolding of an $A_{3}^{i}$ point as given above. Let $U$ be a neighborhood of 0 in $\mathbf{R}^{n}$ and let $\varepsilon>0$. Then there exists a compact neighborhood $B$ of 0 in $U$ and a smooth 
family of embeddings $\phi_{t}: B \rightarrow U, t \in[-\delta, \delta]^{2}$, where $0<\delta<\varepsilon$, so that the following conditions are satisfied.

(1) $\Sigma\left(p_{t}\right) \subset$ int $\phi_{t}(B)$ for all $t \in[-\delta, \delta]^{2}$.

(2) $p_{t} \phi_{t}(x)=g(t) \pm x_{1}^{2}+\sum_{j=2}^{n} \pm x_{j}^{2}$ if either $t_{1}$ is close to $\pm \delta$ or $x$ is close to $\partial B$. Here $g(t)$ is a function only of $t$ and the sign in front of both $x_{1}^{2}$ and $\delta$ is the sign of our $A_{3}$ point.

(3) $p_{t} \phi_{t}(x)$ is invariant under the operation of changing the sign of both $x_{1}$ and $t_{2}$.

(4) For all $g \in S O(1) \times O(i) \times O(n-i-1) \subset O(n)$ and $t \in[-\delta, \delta]^{2}$ we have $g B=B$ and $\phi_{t} g=g \phi_{t}$ and thus $p_{t} \phi_{t} g=p_{t} \phi_{t}$.

Proof. It suffices to prove this in the case $n=1$. Indeed if $B^{1}, \phi_{t}^{1}$ satisfy (1)-(3) in the one dimensional case, then $B=B^{1} \times D, \phi_{t}=\phi_{t}^{1} \times 1_{D}$ satisfies (1)-(4) for the $n$-dimensional case if $D$ is a small disk around 0 in $\mathbf{R}^{n-1}$.

In the one dimensional case it is an elementary exercise to show that $B, \phi_{t}$ exist satisfying (1) and (2). By averaging over the action of $\mathbf{Z}_{2}$ we obtain condition (3). We observe that the average of two orientation preserving embeddings of an interval into $\mathbf{R}$ is again an embedding.

The following is a parameterized version of 3.1 .

Proposition 3.3 (+ Dovetail Lemma). Let $f_{t}: N \rightarrow \mathbf{R}, t \in D^{k}$, be a smooth family of maps which are nonsingular on $N$. Let $S$ be a codimension 1 embedded submanifold of $A_{1}^{i}(f)$ where $i<n$ and suppose that $S$ is compact without boundary. Suppose that for each $z \in S$ there is given a 1-dimensional subspace $L_{z}$ of the $(+)$ eigenspace of $D^{2} f$ at $z$ (with respect to some metric $\mathrm{m}_{t}$ ) so that the line bundle over $S$ formed by the $L_{z}$ 's is isomorphic to the normal bundle of $S$ in $A_{1}^{i}(f)$. Then there is a deformation $f_{t}^{u}$ of $f_{t}$ with support in an arbitrarily small neighborhood of $S$ so that, near $S, f_{t}^{u}$ looks like a twisted product of Figure $\mathrm{E}$ with $S$ (see Figure $\mathrm{H}$ ), where $u=t_{1}$, the normal line to $S$ in $A_{1}^{i}(f)$ gives $t_{2}$ and the line $L$ gives the $x_{1}$-axis. The main effect of this deformation is to cut $A_{1}^{i}(f)$ along $S$ so that $A_{1}^{i}\left(f^{1}\right) \cong A_{1}^{i}(f)-S$.
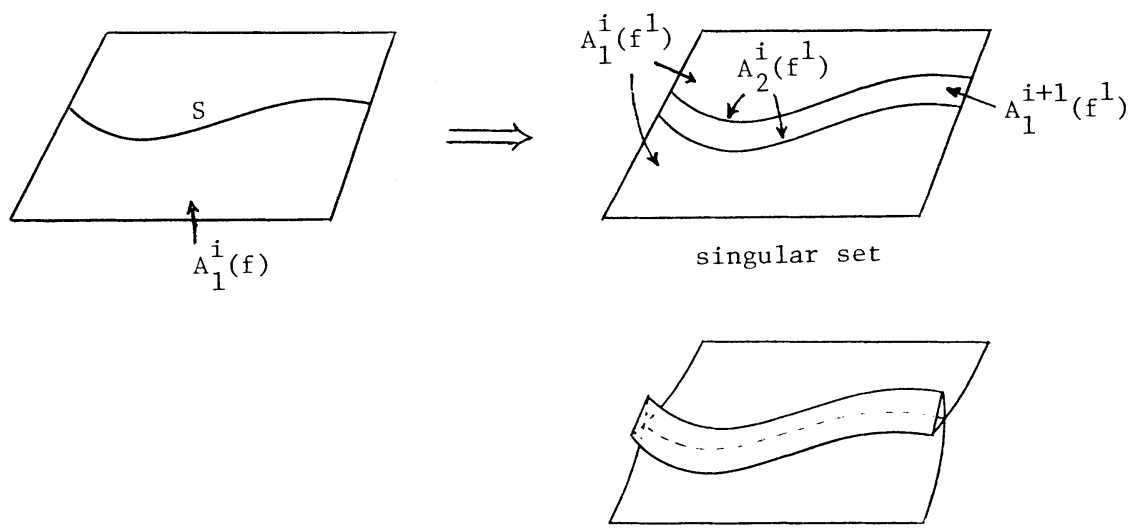

graphic

Figure $\mathrm{H}$ (cutting $A_{1}^{i}(f)$ along $S$ ) 
REMARK 3.4 (-DOVETAIL LEMMA). If we assume that $i \geqslant 1$ and $L_{z}$ lies in the (-) eigenspace of $D^{2} f$ at $z$ for all $z$ in $S$ then we get the analogous statement for negative dovetails.

Proof. We assume that $\nu\left(S, A_{1}^{i}(f)\right)$ is orientable. The nonorientable case follows from the same argument with the observation that our construction is $\mathbf{Z}_{2}$-equivariant (see 3.2(3)). We choose an orientation for $\nu\left(S, A_{1}^{i}(f)\right)$. Given the isomorphism $L=\nu\left(S, A_{1}^{i}(f)\right)$ this gives an orientation for each line $L_{z}$. Let $v_{z}$ be a nonzero vector in $L_{z}$ pointing in the orientation direction. Using the parameterized Morse lemma (1.2) we can make $f_{t}$ look standard along $S$. As in 3.1 we can now paste in the standard unfolding of ${ }^{+} A_{3}^{i}$ using the pasting lemma (3.2) which we made $O(i) \times O(n-i-1)$-equivariant just for this purpose.

Suppose now that $f_{t}: N \rightarrow \mathbf{R}, t \in D^{k}$, is a smooth family of maps nonsingular on $\partial N$ having $A_{1}, A_{2}, A_{3}$ singularities and no others. Suppose that the $A_{3}$ singularities are transverse, i.e. $\tilde{j}^{4} f$ is transverse to $A_{3}(N)$, so that $A_{3}(f)$ is a smooth $(k-2)$ submanifold of $\Sigma(f)$. Then, under certain conditions, $A_{3}(f)$ can be elminated by a nice deformation of $f_{t}$. Suppose that $C$ is a union of components of $A_{3}(f)$. Then the conditions for the elimination of $C$ are as follows (see Figure I).

(a) $C$ is a closed manifold.

(b) $C$ bounds a compact $(k-1)$-submanifold $S \subset \Sigma(f)$.

(c) $C \subset{ }^{\varepsilon} A_{3}^{i}(f)$ where $i$ and $\varepsilon$ are fixed.

(d) int $S \subset A_{1}^{i}(f)$ if $\varepsilon=+$.

(e) int $S \subset A_{1}^{i+1}(f)$ if $\varepsilon=-$.

(f) $S$ meets $A_{2}(f) \cup A_{3}(f)$ transversely along $C$.
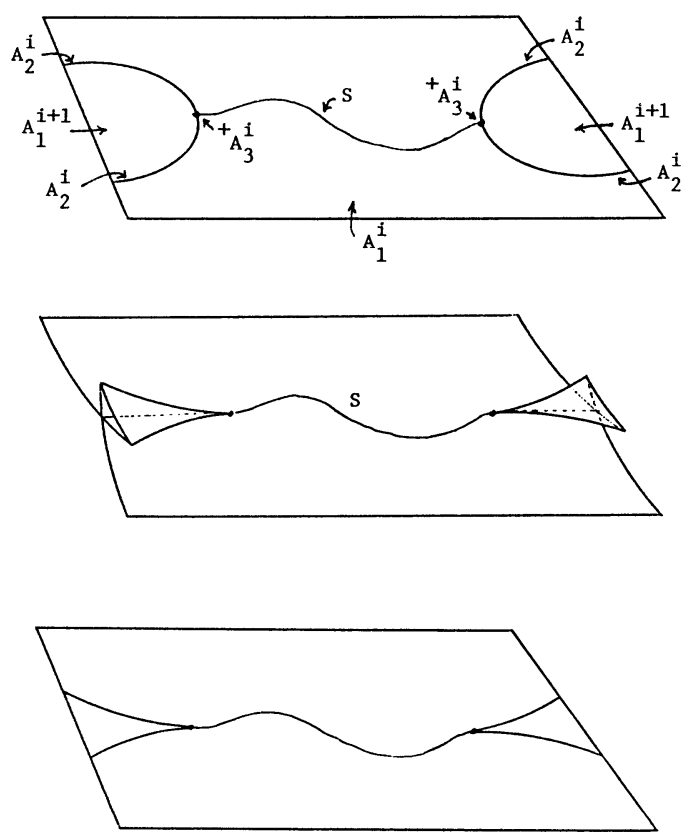

FIGURE I ( ${ }^{+} A_{3}^{i}$ points in cancelling position) 
(g) For each $z \in S$ there is a line $L_{z}$ tangent to $N$ at $z$ so that $L_{z}$ is the kernel of $D^{2} f$ at $z$ when $z \in \partial S=C$ and $L_{z}$ lies in the $\varepsilon$ eigenspace of $D^{2} f$ at $z$ when $z \in$ int $S$.

(h) The line bundle over $S$ formed by the $L_{z}$ 's is isomorphic to the normal bundle of $S$ in $\Sigma(f)$.

Proposition 3.5 (DOVETAIL CANCEllation LEMMA). Under the above conditions there exists a smooth deformation $f_{t}^{u}$ of $f_{t}$ with support in an arbitrarily small neighborhood of $S$ which eliminates the subset $C$ of $A_{3}(f)$ along $S$ as indicated in Figures $\mathrm{J}$ and $\mathrm{K}$. There is one point which is not indicated in the drawing: Along the set $S$ which lies in $A_{1}^{i}(f) \cup A_{3}^{i}(f)$ when $u=0$ and $A_{1}^{i+1}\left(f^{1}\right)$ when $u=1$ (assuming $\varepsilon=+)$ the $(-)$ eigenspace of $D^{2} f$ changes by addition of the tangent lines $L_{z}$.

Proof. First we need a normal form lemma for $A_{3}$ points: The statement and proof is analogous to Lemma 2.2. This makes $f_{t}$ look just like the standard unfolding of an ${ }^{\varepsilon} A_{3}^{i}$ point near $C$. The pasting lemma (3.2) and the parameterized Morse lemma (1.2) can be used to make $f_{t}$ look standard in a neighborhood of $S$. The standard deformation can now be pasted in as shown in Figure L. The dotted lines above $u=1$ indicate that there is no discontinuity at the corners.
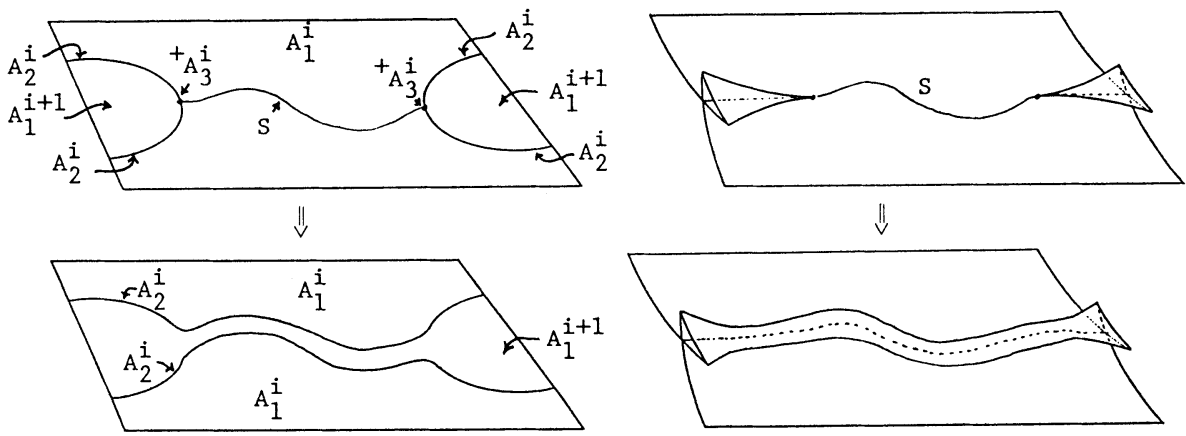

Figure J (cancellation of two ${ }^{+} A_{3}^{i}$ singularities)

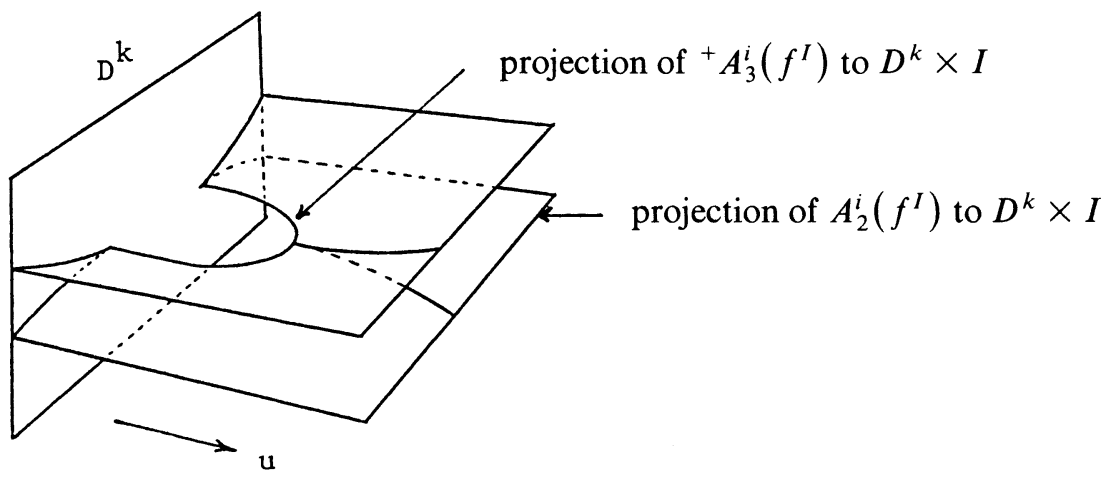

FIGURE K (graphic of the deformation) 


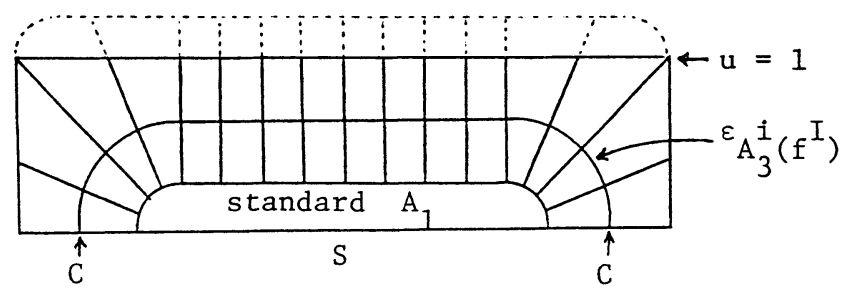

FigURE L (deformation using the standard unfolding of ${ }^{\varepsilon} A_{3}^{i}$ )

A4. Butterflies and $k$-lenses. The standard unfolding of an $A_{4}$ singularity (butterfly) is

$$
p_{t}(x)=x_{1}^{5}+t_{1} x_{1}^{3}+t_{2} x_{1}^{2}+t_{3} x_{1}+\sum_{j=2}^{n} \pm x_{j}^{2}
$$

This gives

$$
\begin{gathered}
\Sigma(p)=\left\{(x, t) \in \mathbf{R}^{n} \times \mathbf{R}^{3} \mid t_{3}=-5 x_{1}^{4}-3 t_{1} x_{1}^{2}-2 t_{2} x_{1}, x_{j}=0 \text { for } j \geqslant 2\right\}, \\
\Sigma^{2}(p)=\left\{(x, t) \in \Sigma(p) \mid t_{2}=-10 x_{1}^{3}-3 t_{1} x_{1}\right\}, \\
\Sigma^{3}(p)=\left\{(x, t) \in \Sigma^{2}(p) \mid t_{1}=-10 x_{1}^{2}\right\} .
\end{gathered}
$$

We use the notation $\Sigma^{k}=A^{k} \cup A^{k+1} \cup \cdots$ in this section $\left(\Sigma^{q}\right.$ denotes the obstruction set in the rest of the paper). Figure $\mathrm{M}$ shows various projections of $\Sigma^{2}(p)$ and $\Sigma^{3}(p)$. This drawing is identical to Figure $\mathrm{E}$ above except for the labels.

We call $t_{1}$ the cancellation direction because two $A_{3}^{i}$ points are being cancelled at the $A_{4}^{i}$ point as $t_{1}$ goes from - to + . As in the last section we shall consider $A_{4}^{i}$ as an instrument for creating (instead of cancelling) pairs of dovetails. Figure $\mathrm{N}$ shows how this works for $p_{t}$ as $t_{1}$ goes from $t_{1}=+1$ to $t_{1}=-1$.
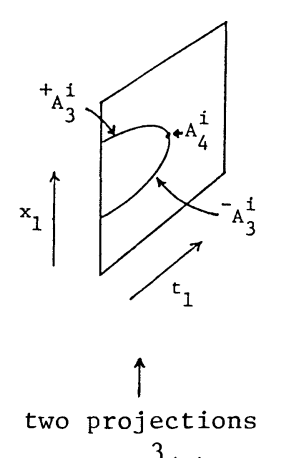

$$
\text { of } \Sigma^{3}(p)
$$
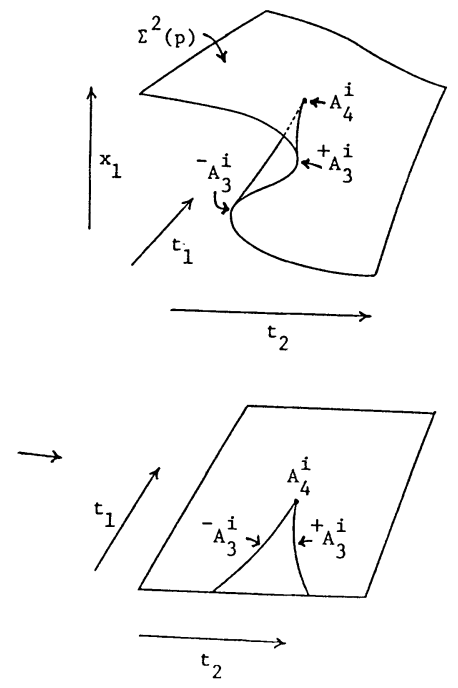

FIGURE M (standard unfolding of $A_{4}^{i}$ )
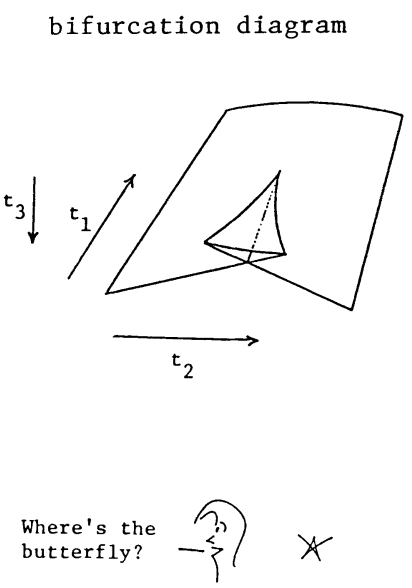

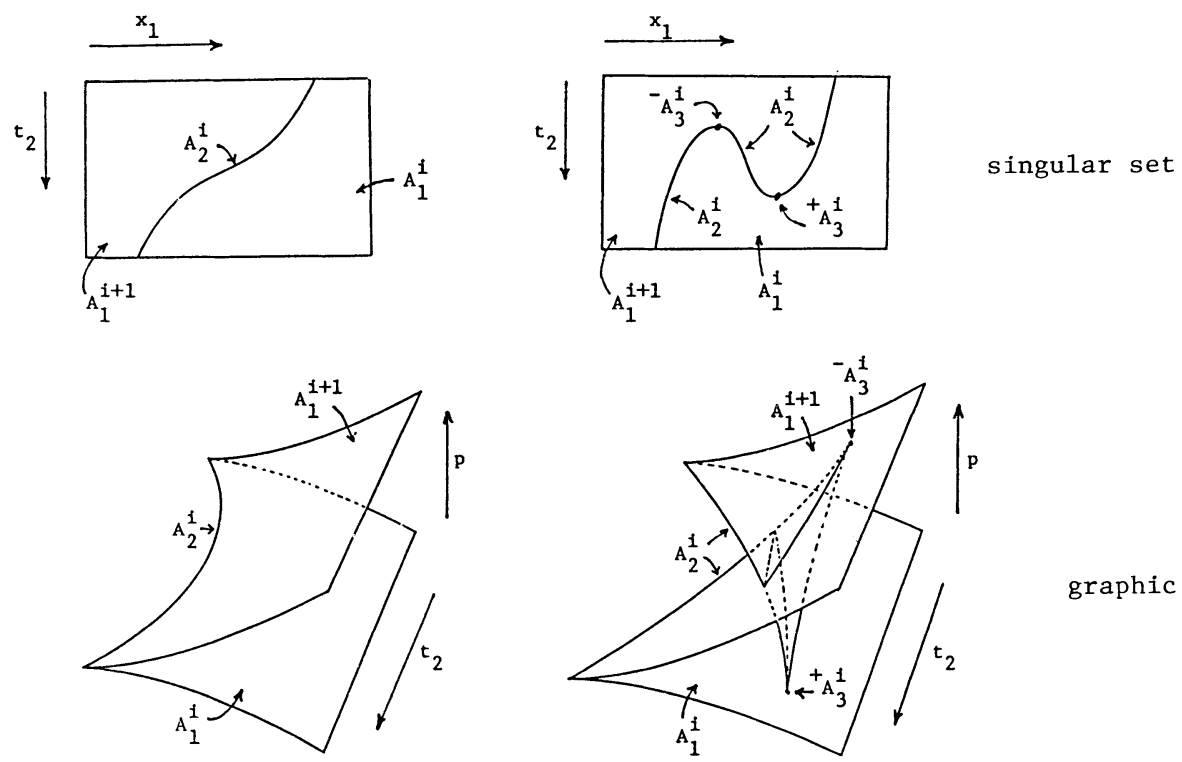

graphic
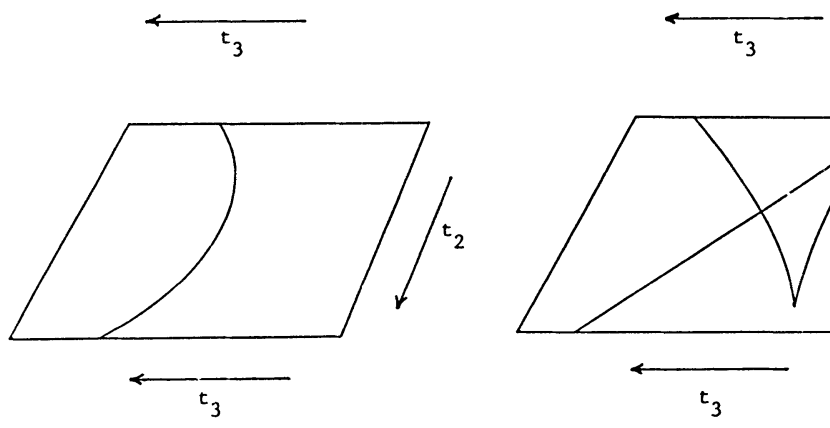

$\left(t_{1}=1\right)$

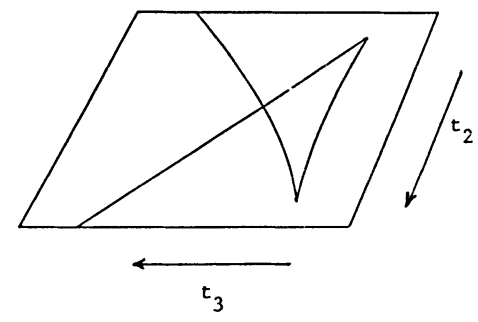

$\left(t_{1}=-1\right)$

FIGURE N ( $A_{4}^{i}$ considered as a deformation)

The simplest case in which the standard unfolding of an $A_{4}$ singularity can be used to create two $A_{3}$ singularities is given in Lemma 4.1 below. This is a baby version of the butterfly lemma (4.3) just as Lemma 3.1 was a baby version of the $(+)$ dovetail lemma (3.3).

LEMMA 4.1. Let $f_{t}: N \rightarrow \mathbf{R}, t \in D^{2}$, be a two parameter family of smooth functions so that $f_{t}$ has no singularities on $\partial N$ and only $A_{1}$ and $A_{2}$ singularities in int $N$ for all $t$. Suppose that all the $A_{2}$ points are transverse and let $\left(x_{0}, t_{0}\right) \in A_{2}^{i}(f) \cap \operatorname{int}\left(N \times D^{2}\right)$. Then there exists a deformation $f_{t}^{u}$ of $f_{t}$ with support in an arbitrarily small neighborhood $U$ of $\left(x_{0}, t_{0}\right)$ so that, inside $U, f_{t}$ looks like the standard unfolding of an $A_{4}^{i}$ singularity as shown in Figure $\mathrm{N}$, where the $t_{2}$ direction can be chosen to correspond to either direction along $A_{2}^{i}(f)$ at $\left(x_{0}, t_{0}\right)$. 
Proof. By the normal form lemma for $A_{2}$ singularities (2.2) we can choose coordinates so that $f_{t}$ looks just like the standard unfolding of an $A_{2}^{i}$ point cross a small interval near $\left(x_{0}, t_{0}\right)$. The standard deformation $p_{t}$ for $A_{4}^{i}$ can now be pasted in using a partition of unity function and the following pasting lemma.

Lemma 4.2 (PASTING LeMma FOR $A_{4}$ POINTS). Let $p_{t}: \mathbf{R}^{n} \rightarrow \mathbf{R}, t \in D^{3}$, be the standard unfolding of an $A_{4}^{i}$ singularity as given above. Let $U$ be a neighborhood of 0 in $\mathbf{R}^{n}$ and let $\varepsilon>0$. Then there exists a compact neighborhood $B$ of 0 in $U, 0<\delta<\varepsilon$, and smooth families of embeddings $\psi_{u}:[-\delta, \delta] \rightarrow(-\varepsilon, \varepsilon), u \in[-\delta, \delta]^{2}$, and $\phi_{t}: B \rightarrow U$, $t \in[-\delta, \delta]^{3}$, so that the following conditions are satisfied. We use the notation $\Psi(t)=\left(t_{1}, t_{2}, \psi_{\left(t_{1}, t_{2}\right)}\left(t_{3}\right)\right)$ and $\Phi_{u}: B \times[-\delta, \delta] \rightarrow U \times(-\varepsilon, \varepsilon), u \in[-\delta, \delta]^{2}, \Phi_{u}(x, v)$ $=\left(\phi_{(u, v)}(x), \psi_{u}(v)\right)$.

(1) $\Sigma\left(p_{\Psi(t)}\right) \subset$ int $\phi_{t}(B)$ for all $t \in[-\delta, \delta]$.

(2) $\Sigma^{2}\left(p_{(u,-)}\right) \subset \operatorname{int} \Phi_{u}(B \times[-\delta, \delta])$ for all $u \in[-\delta, \delta]$.

(3) $p_{\Psi(t)} \phi_{t}(x)=g(t)+x_{1}^{3}+t_{3} x_{1}+\sum_{j=2}^{n} \pm x_{j}^{2}$ if $t_{2}$ is close to $\delta$ or $-\delta, t_{1}$ is close to $\delta$ or $x$ is close to $\partial B$.

(4) For all $g \in S O(1) \times O(i) \times O(n-i-1) \subset O(n)$ and $t \in[-\delta, \delta]^{3}$ we have $g B=B$ and $\phi_{t} g=g \phi_{t}$ and thus $p_{\Psi(t)} \phi_{t} g=p_{\Psi(t)} \phi_{t}$.

Proof. As in Lemma 3.2 it suffices to prove this in the case $n=1$. In this case our lemma follows from elementary (but not necessarily trivial) arguments which we leave to the reader.

The following is the parameterized version of Lemma 4.1. As before we take $f_{t}$ : $N \rightarrow \mathbf{R}, t \in D^{k}$, a smooth family of maps which are nonsingular on $\partial N$ for all $t$ with only $A_{1}$ and transverse $A_{2}$ singularities.

LEMMA 4.3 (BUTTERFLY LEMMA). Let $T$ be a compact codimension 1 submanifold of $A_{2}^{i}(f) \cap \operatorname{int}\left(N \times D^{k}\right)$ without boundary so that the one dimensional normal bundle of $T$ in $A_{2}^{i}(f)$ is trivial (i.e. orientable). Then there exists a deformation $f_{t}^{u}$ of $f_{t}$ with support in an arbitrarily small neighborhood of $T$ which passes through a $(k-2)$ parameter family of $A_{4}^{i}$ points along $T$ and create two dovetail sets ${ }^{+} A_{3}^{i}\left(f^{1}\right)$ and ${ }^{-} A_{3}^{i}\left(f^{1}\right)$ both diffeomorphic to $T$. The deformation looks like (Figure $\left.\mathrm{N}\right) \times T$, where the $t_{2}$ direction can be chosen to be any orientation of $\nu\left(T, A_{2}^{i}(f)\right)$.

Proof. By the normal form lemma for $A_{2}$ points (2.2), $f_{t}$ looks like $T \times$ (the standard two parameter family given on the left side of Figure $\mathrm{N}$ ) with possibly nontrivial bundles $E_{+}, E_{-}$. Since our pasting lemma (4.2) is $O(i) \times O(n-i-1)$ equivariant we can paste in $T \times$ (the standard deformation given in Figure $N$ ).

The next proposition concerns framed $k$-lenses. We recall the definition of a $k$-lens.

Definition 4.4. Let $f_{t}: N \rightarrow \mathbf{R}, t \in D^{k}$, be a $k$-parameter family of maps. Then a $k$-lens (of index $i$ ) is a spherical component $L$ of the critical set $\Sigma(f)$ with the following properties.

(a) $L$ consists of $A_{1}^{i}, A_{1}^{i+1}$ and transverse $A_{2}^{i}$ points. (We write $L_{1}^{i}$ for $A_{1}^{i}(f) \cap L$, etc.)

(b) $L_{2}^{i} \cong S^{k-1}$. 
(c) $L_{1}^{i} \cup L_{2}^{i} \cong D^{k}$.

(d) $L_{1}^{i+1} \cup L_{2}^{i} \cong D^{k}$.

(e) The composition $L_{2}^{i} \subset N \times D^{k} \rightarrow D^{k}$ is an embedding.

Proposition 4.5 (Lens RePlacement lemma). Let $f_{t}$, L be as above. For each $z \in L$ let $v(z)$ be a nonzero vector tangent to $N$ at $z$ which varies smoothly with $z$ and suppose that the following conditions are satisfied.

(1) $v(z)$ lies in the kernel of $D^{2} f(z)$ and points in the positive cubic direction for all $z \in L_{2}^{i}$.

(2) $v(z)$ lies in the $(+)$ eigenspace of $D^{2} f(z)$ for all $z \in L_{1}^{i}$.

(3) $v(z)$ lies in the (-) eigenspace of $D^{2} f(z)$ for all $z \in L_{1}^{i+1}$.

(4) $L$ is null homotopic in $N \times D^{k}$.

(5) $k \leqslant n-2$.

(6) The null homotopy of $L$ in $N \times D^{k}$ gives a trivialization of the tangent bundle of $N$ over $L$. The $(+),(-)$ eigenspaces of $D^{2} f$ and the vector $v(z)$ give a decomposition of $T N \mid L$ into three summands and thus we have a map

$$
L \rightarrow O(n) /(O(i) \times S O(1) \times O(n-i-1)) .
$$

We assume that this map is null homotopic.

Under all these conditions there exists a deformation $f_{t}^{u}$ of $f_{t}$ with the following properties.

(a) $f_{t}^{0}=f_{t}^{1}=f_{t}$.

(b) $\operatorname{supp}\left(f^{u}\right) \cap \Sigma(f)=L$ (i.e. the other critical points of $f_{t}$ are not disturbed).

(c) $\Sigma\left(f^{u}\right)=(\Sigma(f)-L) \times I \amalg D_{0} \amalg D_{1}$, where $D_{0}, D_{1}$ are $(k+1)$-disks with $D_{j}=L \times j$ as indicated in Figure O.

In other words the deformation $f^{u}$ eliminates the original $k$-lens $L$ and replaces it with an identical k-lens (compare $[\mathbf{F}]$ ).

Proof. (This comes from the author's PhD thesis [I1].)

Step 1. We first let $f^{u}$ be the trivial deformation given by $f^{u}=f$ for all $u \in I$. Then we deform $f^{u}$ using dovetails and butterflies.

singular set

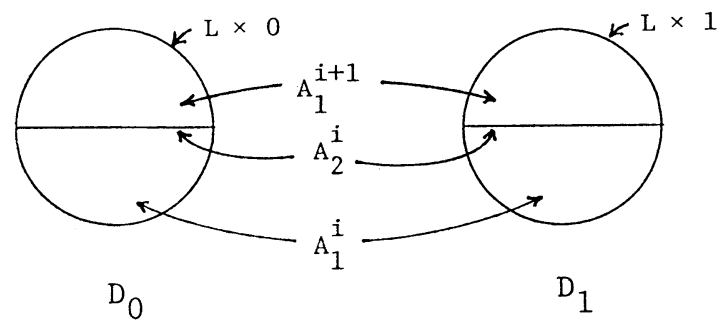

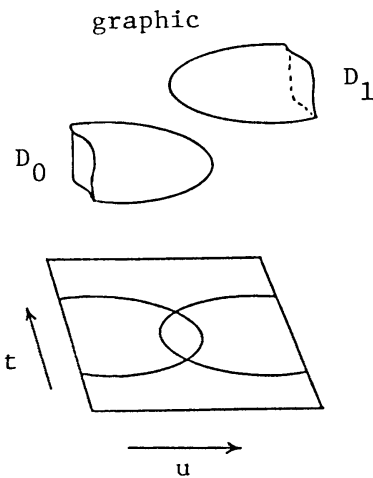

FIGURE O (lens replacement) 
graphic

1)

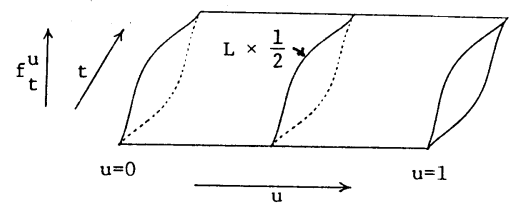

2)

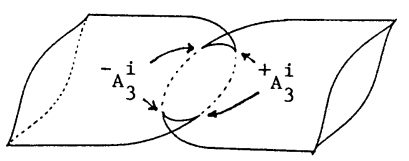

3)

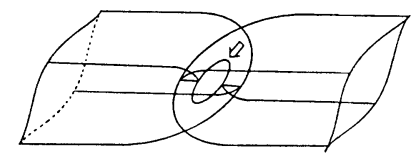

4)

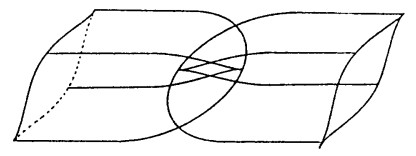

singular set

1)

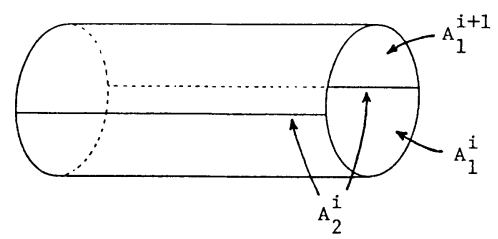

2)

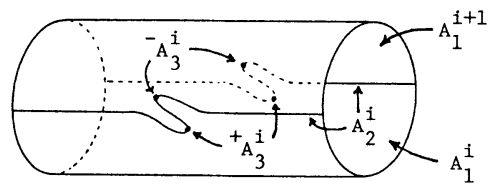

3)

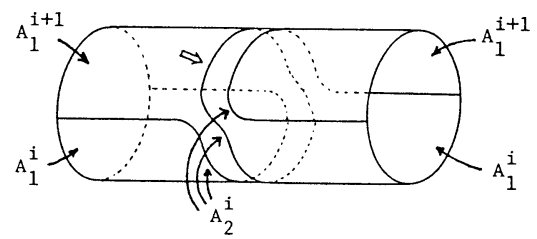

4)

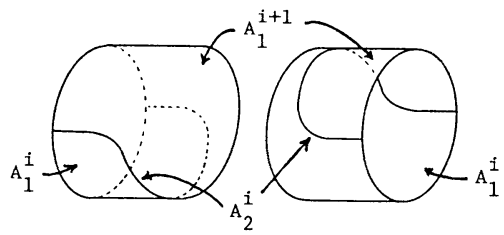

Figure P (proof of lens replacement lemma)

Step 2. At $L_{2}^{i} \times \frac{1}{2} \subset \Sigma\left(f^{u}\right)$ we pass through a $(k-1)$-sphere of butterflies using the butterfly lemma (4.3) and introduce two $(k-1)$-spheres of + and - dovetails as indicated in Figure $\mathrm{P}(2)$.

Step 3. Using the dovetail cancellation lemma (3.5) we eliminate the newly created spheres of ${ }^{+} A_{3}^{i}$ and ${ }^{-} A_{3}^{i}$ points of $f^{u}$ along what is essentially $L_{1}^{i} \times\left(\frac{1}{2}+\varepsilon\right)$ and $L_{1}^{i+1} \times\left(\frac{1}{2}-\varepsilon\right)$ as indicated in Figure $\mathrm{P}(3)$.

Step 4. We have a new $k$-sphere of $A_{2}^{i}$ points which by conditions (5) and (6) satisfy the requirements of the birth-death cancellation lemma (2.3). This gives Figure $\mathrm{P}(4)$ as desired.

The following is the framed version of Proposition 4.5.

Corollary 4.6 (FRAMEd LENS REPlACEMENT LEMMA). Let $\left(f_{t}, \mathfrak{m}_{t}, \xi_{t}\right), t \in D^{k}$, be a transverse family of framed functions on $N$ and let $L$ be a $k$-lens of $f$ of index $i$. If $L$ is null homotopic in $N \times D^{k}$ and $i+k<n$ (and $k \leqslant n-2$ if $i=0$ ), then there is a framed deformation $f^{u}$ of $f$ satisfying (a), (b), (c) of Proposition 4.5.

Proof. Since $\pi_{k} O(n) / O(n-i)=0$ there is an appropriate vector field $v(z)$ so that $v(z)=\xi^{i+1}$ for all $z \in L_{2}^{i} \cup L_{1}^{i+1}$. The deformation $f^{u}$ constructed in the proof of 4.5 has a natural framing if $f$ is framed. 


\section{REFERENCES}

[C] J. Cerf, La stratification naturelle des espaces de fonctions différentiables réelles et le théorème de la pseudo-isotopie, Inst. Hautes Études Sci. Publ. Math. 39 (1970), 5-173.

[F] J. Finney, The invasion of the body snatchers, Dell, New York, 1978.

[G] A. N. Godwin, Three dimensional pictures for Thom's parabolic umbilic, Inst. Hautes Études Sci. Publ. Math. 40 (1971), 117-138.

[H] M. W. Hirsch, Differential topology, Graduate Texts in Math., vol. 33, Springer-Verlag, New York, 1976.

[I1] K. Igusa, The $\mathrm{Wh}_{3}(\pi)$ obstruction for pseudoisotopy, Thesis, Princeton Univ., 1979.

[I2] _ Higher singularities of smooth functions are unnecessary, Ann. of Math. (2) 119 (1984), 1-58.

[13] _ On the homotopy type of the space of generalized Morse functions, Topology 23 (1984), 245-256.

[14] _ The stability theorem for pseudoisotopies (in preparation).

[W] F. Waldhausen, Algebraic K-theory of topological spaces. I, Proc. Sympos. Pure Math., vol. 32, Amer. Math. Soc., Providence, R. I., 1978, pp. 35-60.

DePartment of Mathematics, Brandeis University, Waltham, Massachusetts 02254 